\title{
Non-Gaussian aspects of thermal and kinetic Sunyaev-Zel'dovich effects
}

\author{
Asantha Cooray* \\ Department of Astronomy and Astrophysics, University of Chicago, Chicago, Illinois 60637
}

(Received 26 April 2001; published 28 August 2001)

\begin{abstract}
We discuss non-Gaussian effects associated with the local large-scale structure contributions to the cosmic microwave background (CMB) anisotropies through the thermal Sunyaev-Zel'dovich (SZ) effect. The nonGaussianities associated with the SZ effect arise from the existence of a significant four-point correlation function in large scale pressure fluctuations. Using the pressure trispectrum calculated under the recently popular halo model, we discuss the full covariance of the SZ thermal power spectrum. We use this full covariance matrix to study the astrophysical uses of the SZ effect and discuss the extent to which gas properties can be derived from a measurement of the SZ power spectrum. With the SZ thermal effect separated in temperature fluctuations using its frequency information, the kinetic SZ effect, also known as the OstrikerVishniac effect, is expected to dominate the CMB temperature fluctuations at small angular scales. This effect arises from the baryon modulation of the first order Doppler effect resulting from the relative motion of scatterers. The presence of the SZ kinetic effect can be determined through a cross-correlation between the SZ thermal and a CMB map at small scales. Since the SZ kinetic effect is second order, however, contributions to such a cross-correlation arise to lower order in the form of a three-point correlation function, or a bispectrum in Fourier space. We suggest an additional statistic that can be used to study the correlation between pressure traced by the SZ thermal effect and the baryons traced by the SZ kinetic effect involving the cross-power spectrum constructed through squared temperatures instead of the usual temperature itself. Through a signalto-noise calculation, we show that future small angular scale multifrequency CMB experiments, sensitive to multipoles of a few thousand, will be able to measure the cross-correlation of SZ thermal and SZ kinetic effects through a temperature squared power spectrum.
\end{abstract}

DOI: 10.1103/PhysRevD.64.063514

PACS number(s): 98.80.Es, 95.85.Nv

\section{INTRODUCTION}

In recent years, motivated by the advances in the experimental front, increasing attention has been given to theoretical details related to secondary anisotropies in the cosmic microwave background (CMB) resulting from the low redshift large scale structure. In addition to the direct detection, some of these effects were also studied in detail due to their nonlinear behavior leading to higher order correlations in the CMB temperature fluctuations $[1,2]$. In general, secondary anisotropies resulting from low redshifts ( $z \lesssim$ few tens) can be divided into those that result from gravitational effects and those that result from scattering via free electrons in the reionized epoch.

Through the imparted differential gravitational redshift of CMB photons, when traversing time-evolving potential fluctuations, the integrated Sachs-Wolfe effect [3] contributes at large angular scales. This effect is an important contributor at $z \lesssim 1$ in a cold dark matter model with a cosmological constant $(\Lambda \mathrm{CDM})$ universe. Through angular deflections across the sky via foreground gravitational lensing potentials, weak gravitational lensing modifies the CMB power spectrum at large angular scales while creating power primarily at small angular scales (e.g., Refs. [4-6] and references therein). This lensing effect also correlates with potentials traced by other

\footnotetext{
*Present address: Division of Physics, Mathematics and Astronomy, California Institute of Technology, MS 130-33, Pasadena, California 91125. Email address: asante@hyde.uchicago.edu
}

secondary effects and produces a bispectrum in CMB temperature data (e.g., Refs. [1,2]).

In terms of the scattering effects, the inverse-Compton scattering of CMB photons via hot electrons, the so-called Sunyaev-Zel'dovich (SZ) [7] effect, dominates the small angular scale signal. The SZ effect has now been directly imaged toward massive galaxy clusters (e.g., Refs. [8,9]), where the temperature of the scattering medium can reach as high as $10 \mathrm{keV}$, producing temperature changes in the $\mathrm{CMB}$ of order $1 \mathrm{mK}$ at Rayleigh-Jeans wavelengths. Given that the SZ effect also bears a spectral signature that differs from true temperature fluctuations, the SZ contribution can be separated into multifrequency data, thereby suggesting a means of studying its properties. As discussed in detail in Ref. [10], a multifrequency approach can be applied to current Boomerang [11], and upcoming Microwave Anisotropy Probe ${ }^{1}$ and Planck surveyor ${ }^{2}$ missions.

The improving capabilities of hydrodynamical simulations and analytical models involving a large scale gas distribution have now provided predictions for the SZ effect, mainly the SZ power spectrum and the expected number counts of SZ halos (see, Refs. [10,12-16] among various other studies). A wide-field SZ image, allowing detailed statistical studies such as the angular power spectrum, is yet to be produced, though several experimental attempts are currently in progress for this purpose. These experiments include the interferometric survey at the combined BIMA-

\footnotetext{
${ }^{1}$ http://map.nasa.gsfc.gov

${ }^{2} \mathrm{http}: / /$ astro.estec.esa.nl/Planck/; also, ESA D/SCI(6)3.
} 
OVRO array [CARMA, John Carlstrom (private communication)], the MINT interferometer [Lyman Page (private communication)], and the BOLOCAM array on the Caltech Submillimeter Observatory [Andrew Lange (private communication)].

In the present paper, we further discuss the SZ effect and address what astrophysical properties can be deduced with a measurement of the SZ power spectrum. For this, we require a detailed knowledge of the covariance of the SZ power spectrum beyond the simple Gaussian sample variance. Given that the SZ effect probes the projected pressure distribution in the local universe, its statistical properties reflect those of pressure. As discussed in detail by Cooray [16], the statistics of large scale structure pressure is highly nonGaussian due to the associated nonlinearities. The same nonGaussianities lead to a four-point correlation function in pressure, which in turn, contributes non-negligibly to the power spectrum covariance of the SZ effect.

In order to calculate the covariance associated with SZ power spectrum measurements, we extend the semianalytical model presented by Cooray [16], and calculate the pressure trispectrum. The full covariance matrix now allows us to quantify the astrophysical abilities of SZ measurements as a probe of gas and its temperature properties. Previous to this study, we were unable to perform a similar calculation on how well the SZ effect probes gas and temperature properties due to the unknown covariance associated with the effect. Using a Fisher matrix approach, we quantify to what extent the SZ power spectrum measurements allow a determination of various properties associated with gas and temperature evolution.

Extending our calculation on the contribution of large scale structure gas distribution to $\mathrm{CMB}$ anisotropies through the SZ effect, we also study an associated effect involving baryons associated with halos in the large scale structure. It is well known that the peculiar velocity of galaxy clusters, along the line of sight, also leads to a contribution to temperature anisotropies. This effect is commonly known as the kinetic, or kinematic, Sunyaev-Zel'dovich effect, and arises from the baryon density modulation of the Doppler effect associated with the velocity field [7]. Given that both density and velocity fields are involved, the kinetic SZ effect is essentially second order in density fluctuations; the thermal SZ effect is also second order beacuse of density and temperature dependence. Though the kinetic SZ effect was first described in Ref. [7] using massive galaxy clusters, the same effect was introduced under a different context by Ostriker and Vishniac (OV [19]). The kinetic SZ effect can be considered as the $\mathrm{OV}$ effect extended to the nonlinear regime of baryon fluctuations; however, it should be understood that the basic physical mechanism responsible for the two effects is the same. For the purpose of this presentation, we will treat both the OV effect and the SZ kinetic effect as one contribution, though it may be easier to think of the OV effect as the linear contribution while the kinetic SZ effect, extending to non-linear regime will contain the total contribution. Such a description was provided by Hu in Ref. [20].

We calculate the kinetic SZ-OV effect, hereafter simply referred to as the kinetic SZ effect, using the model we de- veloped to study the thermal SZ effect. We further extend this calculation to consider the correlation between SZ thermal and SZ kinetic effects. Since there is no first order crosscorrelation, the lowest order contribution to the correlation comes from a three-point function, or a bispectrum. Cooray and $\mathrm{Hu}$ discussed this bispectrum in Ref. [2]. Here, we consider an additional possibility of measuring the SZ thermalkinetic cross-correlation via a two-point correlation function which involves squares of the temperature instead of the usual temperature itself. The power spectrum of squared temperatures probes one aspect of the trispectrum resulting from the pressure-baryon cross-correlation. Here we show that there is an adequate signal-to-noise ratio for a reliable measurement of the SZ thermal-SZ kinetic squared power spectrum measurement in upcoming small angular scale experiments.

The layout of the paper is as follows. In Sec. II, we review the background material relevant for current calculations on SZ thermal and SZ kinetic effects in the context of the adiabatic cold dark matter (CDM) models and using clustered dark matter halos as a way to calculate non-linear clustering properties. In Sec. III, we discuss the pressure trispectrum and the covariance of pressure fluctuations in the local universe. In Sec. IV, we detail the calculation of the SZ effect due to large scale structure gas distribution, and calculate its trispectrum. In the same section, using a Fisher matrix formulation, we use the full covariance, including nonGaussianities, of binned SZ power spectrum measurements to establish the astrophysical uses of the SZ effect. The kinetic SZ effect is discussed in Sec. V, and we study the cross-correlation between SZ thermal and SZ kinetic effects in the form of a bispectrum and the power spectrum of squared temperatures in Sec. VI. We conclude in Sec. VII with a summary.

\section{GENERAL DERIVATION}

We first review the properties of adiabatic CDM models relevant to the present calculations. We then discuss the general properties of the halo model as applied to the calculation of the nonlinear dark matter, baryon and pressure density field power spectra of the local large scale structure.

\section{A. Adiabatic CDM model}

The expansion rate for adiabatic CDM cosmological models with a cosmological constant is

$$
H^{2}=H_{0}^{2}\left[\Omega_{m}(1+z)^{3}+\Omega_{K}(1+z)^{2}+\Omega_{\Lambda}\right],
$$

where $H_{0}$ can be written as the inverse Hubble distance today $H_{0}^{-1}=2997.9 h^{-1} \mathrm{Mpc}$. We follow the conventions that in units of the critical density $3 H_{0}^{2} / 8 \pi G$, the contribution of each component is denoted $\Omega_{i}, i=c$ for the CDM, $g$ for the baryons, and $\Lambda$ for the cosmological constant. We also define the auxiliary quantities $\Omega_{m}=\Omega_{c}+\Omega_{g}$ and $\Omega_{K}=1-\Sigma_{i} \Omega_{i}$, which represent the matter density and the contribution of spatial curvature to the expansion rate respectively. 
Convenient measures of distance and time include the conformal distance (or lookback time) from the observer at redshift $z=0$,

$$
r(z)=\int_{0}^{z} \frac{d z^{\prime}}{H\left(z^{\prime}\right)}
$$

and the analogous angular diameter distance

$$
d_{A}=H_{0}^{-1} \Omega_{K}^{-1 / 2} \sinh \left(H_{0} \Omega_{K}^{1 / 2} r\right) .
$$

Note that as $\Omega_{K} \rightarrow 0, d_{A} \rightarrow r$ and we define $r(z=\infty)=r_{0}$.

The adiabatic CDM model possesses two-, three- and four-point correlations of the dark matter density field as defined in the usual way:

$$
\begin{gathered}
\left\langle\delta\left(\mathbf{k}_{1}\right) \delta\left(\mathbf{k}_{2}\right)\right\rangle=(2 \pi)^{3} \delta_{\mathrm{D}}\left(\mathbf{k}_{12}\right) P\left(k_{1}\right), \\
\left\langle\delta\left(\mathbf{k}_{1}\right) \delta\left(\mathbf{k}_{2}\right) \delta\left(\mathbf{k}_{3}\right)\right\rangle=(2 \pi)^{3} \delta_{\mathrm{D}}\left(\mathbf{k}_{123}\right) B\left(\mathbf{k}_{1}, \mathbf{k}_{2}, \mathbf{k}_{3}\right), \\
\left\langle\delta\left(\mathbf{k}_{1}\right) \ldots \delta\left(\mathbf{k}_{4}\right)\right\rangle_{c}=(2 \pi)^{3} \delta_{\mathrm{D}}\left(\mathbf{k}_{1234}\right) T\left(\mathbf{k}_{1}, \mathbf{k}_{2}, \mathbf{k}_{3}, \mathbf{k}_{4}\right),
\end{gathered}
$$

where $\mathbf{k}_{i \ldots j}=\mathbf{k}_{i}+\cdots+\mathbf{k}_{j}$, and $\delta_{\mathrm{D}}$ is the delta function not to be confused with the density perturbation. Note that the subscript $c$ denotes the connected piece, i.e. the trispectrum is defined to be identically zero for a Gaussian field. Here and throughout, we occasionally suppress the redshift dependence where no confusion will arise.

In linear perturbation theory, ${ }^{3}$

$$
\left.\frac{k^{3} P^{\operatorname{lin}}(k)}{2 \pi^{2}}\right|_{z=0}=\delta_{H}^{2}\left(\frac{k}{H_{0}}\right)^{n+3} T^{2}(k) .
$$

We use the fitting formulas of Eisenstein and $\mathrm{Hu}$ [21] to evaluate the transfer function $T(k)$ for CDM models. Here $\delta_{H}$ is the amplitude of present-day density fluctuations at the Hubble scale.

The bispectrum in perturbation theory is given by ${ }^{4}$

$$
B^{\operatorname{lin}}\left(\mathbf{k}_{p}, \mathbf{k}_{q}, \mathbf{k}_{r}\right)=2 F_{2}^{\mathrm{s}}\left(\mathbf{k}_{p}, \mathbf{k}_{q}\right) P\left(k_{p}\right) P\left(k_{q}\right)+2 \text { Perm., }
$$

with $F_{2}^{\mathrm{s}}$ term given by second order gravitational perturbation calculations.

Similarly, the perturbation theory trispectrum follows from Fry [23]:

\footnotetext{
${ }^{3}$ It should be understood that "lin" denotes here the lowest nonvanishing order of perturbation theory for the object in question. For the power spectrum, this is linear perturbation theory; for the bispectrum, this is second order perturbation theory, etc.

${ }^{4}$ The kernels $F_{n}^{\mathrm{s}}$ are derived in Ref. [22] [see, Eqs. (A2) and (A3) of Ref. [22]; note that $P_{n} \equiv F_{n}$ ], and we have written such that the symmetric form of $F_{n}$ 's are used. The use of the symmetric form accounts for the factor of 2 in Eq. (8) and factors of 4 and 6 in Eq. (9).
}

$$
\begin{aligned}
T^{\text {lin }}= & 6\left[F_{3}^{\mathrm{s}}\left(\mathbf{k}_{1}, \mathbf{k}_{2}, \mathbf{k}_{3}\right) P\left(k_{1}\right) P\left(k_{2}\right) P\left(k_{3}\right)+\text { Perm. }\right] \\
& +4\left[F_{2}^{\mathrm{s}}\left(\mathbf{k}_{12},-\mathbf{k}_{1}\right) F_{2}^{\mathrm{s}}\left(\mathbf{k}_{12}, \mathbf{k}_{3}\right) P\left(k_{1}\right) P\left(k_{12}\right) P\left(k_{3}\right)\right. \\
& + \text { Perm. }] .
\end{aligned}
$$

The permutations involve a total of four terms in the first set and 12 terms in the second set.

In linear theory, the density field may be scaled backwards to a higher redshift by the use of the growth function $G(z)$, where $\delta(k, r)=G(r) \delta(k, 0)[24]$ :

$$
G(r) \propto \frac{H(r)}{H_{0}} \int_{z(r)}^{\infty} d z^{\prime}\left(1+z^{\prime}\right)\left(\frac{H_{0}}{H\left(z^{\prime}\right)}\right)^{3} .
$$

Note that in the matter dominated epoch $G \propto a=(1+z)^{-1}$.

The continuity equation relates the density and velocity fields, in the linear regime, via

$$
\mathbf{v}=-i \dot{G} \delta(k, 0) \frac{\mathbf{k}}{k^{2}},
$$

where overdots represent derivatives with respect to radial distance $r$. In this paper, we ignore the contribution to the velocity field within individual nonlinear structures, including a curl component, and only consider this potential bulk flow. This large scale velocity field is independent of the nonlinear density fluctuations, and thus there is no correlation between this bulk flow and the density field within halos.

For fluctuation spectra and growth rates of interest here, reionization of the universe is expected to occur rather late $z_{\mathrm{ri}} \leqslant 50$ such that the reionized media is optically thin to Thomson scattering of CMB photons $\tau \lesssim 1$. The probability of last scattering within $d r$ of $r$ (the visibility function) is

$$
g=\dot{\tau} e^{-\tau}=X H_{0} \tau_{H}(1+z)^{2} e^{-\tau} .
$$

Here $\tau(r)=\int_{0}^{r} d r \dot{\tau}$ is the optical depth out to $r, X$ is the ionization fraction, and

$$
\tau_{H}=0.0691\left(1-Y_{p}\right) \Omega_{g} h
$$

is the optical depth to Thomson scattering to the Hubble distance today, assuming full hydrogen ionization with primordial helium fraction of $Y_{p}$. Note that the ionization fraction can exceed unity: $X=\left(1-3 Y_{p} / 4\right) /\left(1-Y_{p}\right)$ for singly ionized helium and $X=\left(1-Y_{p} / 2\right) /\left(1-Y_{p}\right)$ for fully ionized helium.

Although we maintain generality in all derivations, we illustrate our results with the currently favored $\Lambda \mathrm{CDM}$ cosmological model. The parameters for this model are $\Omega_{c}$ $=0.30, \Omega_{g}=0.05, \Omega_{\Lambda}=0.65, h=0.65, Y_{p}=0.24, n=1$, and $X=1$, with a normalization such that mass fluctuations on the $8 \mathrm{~h} \mathrm{Mpc}^{-1}$ scale is $\sigma_{8}=0.9$, consistent with observations on the abundance of galaxy clusters [25] and Cosmic Background Explorer (COBE) normalization [26]. A reasonable value here is important since higher order correlations are nonlinearly dependent on the amplitude of the density field. 
For the Sunyaev-Zel'dovich effects discussed here, we are more interested in the clustering properties of pressure and baryons, rather than the dark matter density field. We do not have a reliable way to calculate the pressure, baryon power spectra and their higher order correlations and crosscorrelations analytically. We will introduce the semianalytic halo model for this purpose following [16].

\section{B. Halo approach}

In order to calculate the nonlinear clustering of the dark matter, baryon and pressure density fields, we use the halo model described in Refs. [27-29]. Underlying the halo approach is the assertion that dark matter halos of virial mass $M$ are locally biased tracers of density perturbations in the linear regime. In this case, functional relationship between the overdensity of halos and mass can be expanded in a Taylor series

$$
\begin{aligned}
\delta_{\mathrm{h}}(\mathbf{x}, M ; z)= & b_{0}+b_{1}(M ; z) \delta_{\operatorname{lin}}(\mathbf{x} ; z)+\frac{1}{2} b_{2}(M ; z) \delta_{\operatorname{lin}}^{2}(\mathbf{x} ; z) \\
& +\cdots
\end{aligned}
$$

The over-density of halos can be related to more familiar mass function and the halo density profile by noting that we can model the fully nonlinear density field as a set of correlated discrete objects or halos with profiles

$$
\rho(\mathbf{x})=\sum_{i} \rho_{\mathrm{h}}\left(\mathbf{x}-\mathbf{x}_{i} ; M_{i}\right),
$$

where the sum is over all halo positions. The density fluctuation in Fourier space is

$$
\delta(\mathbf{k})=\sum_{i} e^{i \mathbf{k} \cdot \mathbf{x}_{i}} \delta_{\mathrm{h}}\left(\mathbf{k}, M_{i}\right) .
$$

Following Peebles in Ref. [24], we divide space into sufficiently small volumes $\delta V$ that they contain only one or zero halos of a given mass, and convert the sum over halos to a sum over the volume elements and masses:

$$
\delta(\mathbf{k})=\sum_{V_{1}, M_{1}} n_{1} e^{i \mathbf{k} \cdot \mathbf{x}_{1}} \delta_{\mathrm{h}}\left(\mathbf{k}, M_{1}\right) .
$$

By virtue of the small volume element $n_{1}=n_{1}^{2}=n_{1}^{\mu}=1$ or 0 following Ref. [24].

Using the fact that halos are biased tracers of the density field such that their number density fluctuates as

$$
\begin{aligned}
\frac{d n}{d M}(\mathbf{x} ; z)= & \frac{d \bar{n}}{d M}(M ; z)\left[b_{0}+b_{1}(M ; z) \delta_{\operatorname{lin}}(\mathbf{x} ; z)\right. \\
& \left.+\frac{1}{2} b_{2}(M ; z) \delta_{\operatorname{lin}}^{2}(\mathbf{x} ; z) \ldots\right]
\end{aligned}
$$

we can write

$$
\begin{gathered}
\left\langle n_{1}\right\rangle=\frac{d \bar{n}}{d M_{1}} \delta M_{1}, \\
\left\langle n_{1} n_{2}\right\rangle=\left\langle n_{1}\right\rangle^{2} \delta_{12}+\left\langle n_{1}\right\rangle\left\langle n_{2}\right\rangle\left[b_{0}^{2}+b_{1}\left(M_{1}\right) b_{1}\left(M_{2}\right),\right. \\
\left.\times\left\langle\delta_{\operatorname{lin}}\left(\mathbf{x}_{1}\right) \delta_{\operatorname{lin}}\left(\mathbf{x}_{2}\right)\right\rangle\right], \\
\left\langle n_{1} n_{2} n_{3}\right\rangle=\cdots
\end{gathered}
$$

In Eq. (18), $b_{0} \equiv 1$ and $\delta_{12}$ is the Dirac delta function, and we have included only the lowest order terms. The halo bias parameters are given in Ref. [30]:

$$
b_{1}(M ; z)=1+\frac{\nu^{2}(M ; z)-1}{\delta_{c}}
$$

and

$$
b_{2}(M ; z)=\frac{8}{21}\left[b_{1}(M ; z)-1\right]+\frac{\nu^{2}(M ; z)-3}{\sigma^{2}(M ; z)} .
$$

Here $\nu(M ; z)=\delta_{c} / \sigma(M ; z), \sigma(M ; z)$ is the rms fluctuation within a top-hat filter at the virial radius corresponding to mass $M$, and $\delta_{c}$ is the threshold overdensity of spherical collapse (see Ref. [31] for useful fitting functions). We ignore terms involving cubic and higher order bias terms as these only make contributions at a level less than $1 \%$.

The derivation of the higher point functions in Fourier space is now a straightforward but tedious exercise in algebra (see Ref. [46]). The Fourier transforms inherent in Eq. (17) convert the correlation functions in Eq. (20) into the power spectrum, bispectrum, trispectrum, etc., of perturbation theory.

Following Refs. [28] and [16], it is now convenient to define a general integral over the halo mass function $d \bar{n} / d M$. Though we presented a description of halo clustering for dark matter, we can generalize this discussion to consider any physical property associated with a halo; one simply relates the overdensity of halos through the density profile corresponding to the property of interest in Eq. (14). Since we will encounter dark matter, pressure and baryon density fields throughout this paper, we write a general integral that applies to all three properties:

$$
\begin{aligned}
I_{\mu, i_{1} \ldots i_{\mu}}^{\beta, \eta}\left(k_{1}, \ldots,\right. & \left.k_{\mu} ; z\right) \\
\equiv & \int d M \frac{d \bar{n}}{d M}(M, z) b_{\beta}(M ; z) \\
& \times T_{e}(M ; z)^{\eta} y_{i_{1}}\left(k_{1}, M ; z\right) \ldots y_{i_{\mu}}\left(k_{\mu}, M ; z\right) .
\end{aligned}
$$

Here, in addition to the dark matter, to account for clustering properties of pressure associated with baryons in large scale structure, we have introduced the electron temperature $T_{e}(M ; z)$.

In Eq. (23), the three-dimensional Fourier transform of the density fluctuation, through the halo profile of the density distribution of any physical property $\rho_{i}(r, M ; z)$, is 


$$
y_{i}(k, M ; z)=\frac{1}{\rho_{b i}} \int_{0}^{r_{v}} d r 4 \pi r^{2} \rho_{i}(r, M ; z) \frac{\sin (k r)}{k r} \text {, }
$$

with the background mean density of the same quantity given by $\rho_{b i}$. Since in this paper we discuss dark matter, pressure and baryons, the index $i$ will be used to represent the density $\delta$ (with $y \equiv y_{\delta}$ ), the baryons $g$ (with $y \equiv y_{g}$ ), or the pressure $\Pi$ (with $\equiv y_{g}$ ). Note that for both dark matter density and baryon clustering, $\eta=0$, as there is no temperature contribution, but for clustering of pressure, $\eta=\mu$ when $i_{1} \ldots i_{\mu}$ describes pressure; therefore, we will no longer make use of the index $\eta$ in the following discussion. One additional note here is that the profile used for baryons will be the same as the profile that we will use for pressure. The only difference between baryon clustering and pressure clustering is that we weigh the latter with the electron temperature, leading to a selective contribution from electrons with the highest temperature, while the former includes all baryons.

\section{Ingredients}

The dark matter profile of collapsed halos are taken to be the Navarrow-Frenk-White (NFW) [32] halos with a density distribution

$$
\rho_{\delta}(r)=\frac{\rho_{s}}{\left(r / r_{s}\right)\left(1+r / r_{s}\right)^{2}} .
$$

The density profile can be integrated and related to the total dark matter mass of the halo within $r_{v}$,

$$
M_{\delta}=4 \pi \rho_{s} r_{s}^{3}\left[\log (1+c)-\frac{c}{1+c}\right],
$$

where the concentration $c$ is $r_{v} / r_{s}$. Choosing $r_{v}$ as the virial radius of the halo, spherical collapse tells us that $M$ $=4 \pi r_{v}^{3} \Delta(z) \rho_{b} / 3$, where $\Delta(z)$ is the overdensity of collapse and $\rho_{b}$ is the background matter density today. We use comoving coordinates throughout. By equating these two expressions, one can eliminate $\rho_{s}$ and describe the halo by its mass $M$ and concentration $c$. We use the Press-Schechter [33] mass function to describe the halo mass function written as $d \bar{n} / d M(M ; z)$. Following the results from $\Lambda \mathrm{CDM}$ simulations in Ref. [34], we take a concentration-mass relationship which is consistent with the mean

$$
c(M ; z)=9(1+z)^{-1}\left[\frac{M}{M_{\star}(z)}\right]^{-0.13},
$$

where $M_{\star}(z)$ is the nonlinear mass scale at which the peakheight threshold $\nu(M ; z)$ is equal to 1 . Due to computational limitations, we ignore the distribution of concentrations, for a given mass, observed in simulations by Ref. [34] and only use the mean value. As discussed in Ref. [18], the concentration distribution is important for higher moments and ignoring the distribution in present work, it is likely that we underestimate the non-Gaussian contribution from the single halo term at a level of $20 \%$. We also expect uncertainties at the same level due to our modeling of halos as smooth spherical distributions and by ignoring the substructure and asphericity of halos.

The gas density profile $\rho_{g}(r)$ is calculated assuming a hydrostatic equilibrium between the gas distribution and the dark matter density field within a halo. This is a valid assumption given that current observations of halos, mainly galaxy clusters, suggest the existence of regularity relations, such as size temperature (e.g., Ref. [35]), between physical properties of dark matter and baryon distributions. Using the hydrostatic equilibrium allows us to write the baryon density profile $\rho_{g}(r)$ within halos as

$$
\rho_{g}(r)=\rho_{g 0} e^{-b}\left(1+\frac{r}{r_{s}}\right)^{b r_{s} / r}
$$

where $b$ is a constant, for a given mass, and

$$
b=\frac{4 \pi G \mu m_{p} \rho_{s} r_{s}^{2}}{k_{B} T_{e}},
$$

with the Boltzmann constant $k_{B}$ [36] and $\mu=0.59$, corresponding to a hydrogen mass fraction of $76 \%$. Equation (29) is derived only under the assumption of hydrostatic equilibrium for the gas distribution in a dark matter profile given by the NFW equation. The hydrostatic equilibrium allows a basic physical assumption to relate pressure and dark matter. Though effects such as mergers between halos are expected to result in a violation of this assumption, we do not consider variants since previous predictions made with the above halo were found to be consistent with numerical simulations by Refregier and Teyssier [15]. Above, the normalization $\rho_{g o}$ is determined under the assumption of a constant gas mass fraction for halos comparable with the universal baryon to dark matter ratio: $f_{g} \equiv M_{g} / M_{\delta}=\Omega_{g} / \Omega_{m}$. When investigating astrophysical uses of the SZ effect, we will vary this parameter and consider variations of gas fraction as a function of mass and redshift.

The electron temperature can be calculated based on the virial theorem or similar arguments as discussed in Ref. [16]. Using the virial theorem, we can write

$$
k_{B} T_{e}=\frac{\gamma G \mu m_{p} M_{\delta}}{3 r_{v}},
$$

with $\gamma=3 / 2$. Since $r_{v} \propto M_{\delta}^{1 / 3}(1+z)^{-1}$ in physical coordinates, $T_{e} \propto M^{2 / 3}(1+z)$. The average density weighted temperature is

$$
\left\langle T_{e}\right\rangle_{\delta}=\int d M \frac{M}{\rho_{b}} \frac{d n}{d M}(M, z) T_{e}(M, z) .
$$
is

The total gas mass present in a dark matter halo within $r_{v}$

$$
M_{g}\left(r_{v}\right)=4 \pi \rho_{g 0} e^{-b} r_{s}^{3} \int_{0}^{c} d x x^{2}(1+x)^{b / x}
$$

In Fig. 1, we show the NFW profile for the dark matter and arbitrarily normalized gas profiles predicted by the hy- 


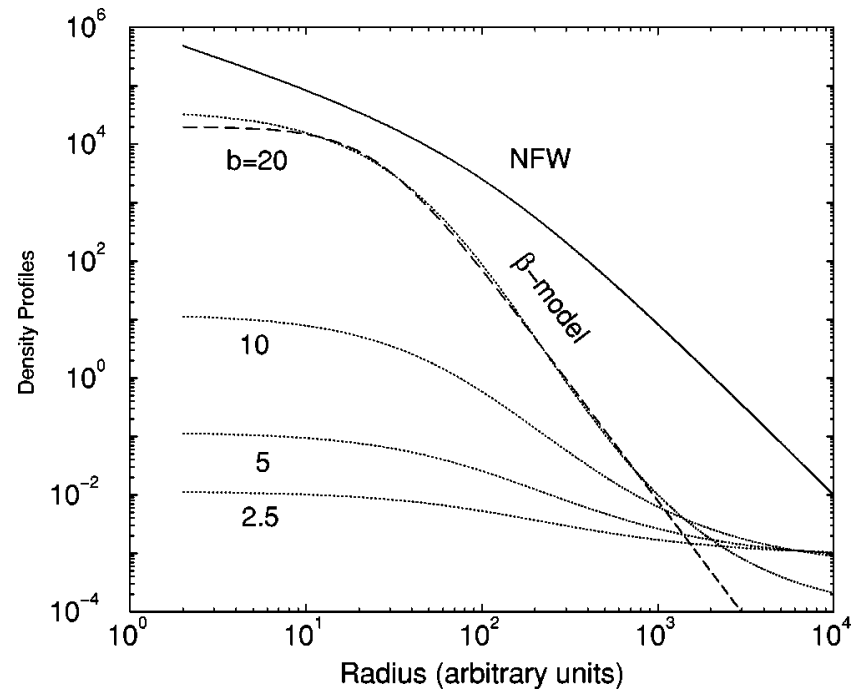

FIG. 1. The dark matter (NFW) profile and the profiles predicted by the hydrostatic equilibrium for gas, as a function of the $b$ parameter [see Eq. (29)] with $r_{s}=100$. For comparison, we also show a typical example of the so-called $\beta$ model $\left(1+r^{2} / r_{c}^{2}\right)^{-3 \beta / 2}$ which is generally used as a fitting function for $\mathrm{x}$-ray and $\mathrm{SZ}$ observations of clusters.

drostatic equilibrium and virial theorem for several values of $b$. As $b$ is decreased, such that the temperature is increased, since $b \propto 1 / T_{e}$, the turnover radius of the gas distribution shifts to higher radii. The relative normalization of the gas profiles are set with a gas fraction value of 0.1 , while the NFW profile is arbitrarily normalized with $\rho_{s}=1$; the gas profiles, therefore, simply scale with the same factor related to true value of $\rho_{s}$. As an example, we also show the socalled $\beta$ model that is commonly used to describe $\mathrm{x}$-ray and SZ observations of galaxy clusters and for the derivation purpose of the Hubble constant by combined SZ or x-ray data. The $\beta$ model quite accurately describes the underlying gas distribution predicted by the gas profile in Eq. (28), though, we find differences at the outermost radii of halos. This difference can be used as a way to establish the hydrostatic equilibrium for clusters with respect to a NFW like dark matter distribution, though, any difference of gas distribution at large radii may need to be accounted for in the context of possible substructure and mergers.

A discussion on the comparison between the gas profile used here and the $\beta$ model is available in Ref. [36]. In addition, we refer the reader to Ref. [16] for a full detailed discussion on issues related to modeling of pressure power spectrum using halos and associated systematic errors. Comparisons of the halo model predictions with numerical simulations are available in Refs. [13] and [15]. Similarly, issues related to modeling of the dark matter clustering using halos are discussed in Ref. [28] for the bispectrum and Ref. [18] for the trispectrum.

\section{Power spectra and trispectra}

For the calculations presented in this paper, we will encounter the power spectrum and trispectrum involving dark matter, pressure and baryons and their cross-correlations. We now write down the relevant Fourier space correlations under the halo approach. To generalize the discussion, we will use the index $i$ to represent the property of interest.

In general, the power spectra of these three quantities under the halo model now become [27]

$$
\begin{aligned}
P_{i}(k) & =P_{i}^{1 h}(k)+P_{i}^{2 h}(k), \\
P_{i}^{1 h}(k) & =I_{2, i i}^{0}(k, k), \\
P_{i}^{2 h}(k) & =\left[I_{1, i}^{1}(k)\right]^{2} P^{\operatorname{lin}}(k),
\end{aligned}
$$

where the two terms represent contributions from two points in a single halo $(1 h)$ and points in two different halos $(2 h)$ respectively.

Similar to above, we can also define the cross power spectra between two fields $i$ and $j$ as

$$
\begin{aligned}
P_{i j}(k) & =P_{i j}^{1 h}(k)+P_{i j}^{2 h}(k), \\
P_{i j}^{1 h}(k) & =I_{2, i j}^{0}(k, k), \\
P_{i j}^{2 h}(k) & =I_{1, i}^{1}(k) I_{1, j}^{1}(k) P^{\operatorname{lin}}(k) .
\end{aligned}
$$

It is also useful to define the bias of one field relative to the dark matter density field as

$$
\operatorname{bias}_{i}(k)=\sqrt{\frac{P_{i}(k)}{P_{\delta}(k)}} .
$$

We can also define a dimensionless correlation coefficient between the two fields as

$$
r_{i j}(k)=\frac{P_{i j}(k)}{\sqrt{P_{i}(k) P_{j}(k)}} .
$$

During the course of this paper, we will encounter, and use, cross-power spectra such as those involving the baryon and the pressure, $P_{g \Pi}$ and the dark matter and the pressure, $P_{\delta \Pi}$.

As described in Ref. [18], the contributions to the trispectrum may be separated into those involving 1-4 halos,

$$
T_{i}=T_{i}^{1 h}+T_{i}^{2 h}+T_{i}^{3 h}+T_{i}^{4 h},
$$

where here and below the argument of the trispectrum is understood to be $\left(\mathbf{k}_{1}, \mathbf{k}_{2}, \mathbf{k}_{3}, \mathbf{k}_{4}\right)$. The term involving a single halo probes correlations of the property of interest, e.g., dark matter, within that halo,

$$
T_{i}^{1 h}=I_{4, i i i i}^{0}\left(k_{1}, k_{2}, k_{3}, k_{4}\right),
$$

and is independent of configuration due to the assumed spherical symmetry for our halos.

The term involving two halos can be further broken up into two parts,

$$
T_{i}^{2 h}=T_{31, i i i i}^{2 h}+T_{22, i i i i}^{2 h},
$$

which represent taking three or two points in the first halo: 


$$
\begin{aligned}
& T_{31, i i i i}^{2 h}=P^{\operatorname{lin}}\left(k_{1}\right) I_{3, i i i}^{1}\left(k_{2}, k_{3}, k_{4}\right) I_{1, i}^{1}\left(k_{1}\right)+3 \text { Perm., } \\
& T_{22, i i i i}^{2 h}=P^{\operatorname{lin}}\left(k_{12}\right) I_{2, i i}^{1}\left(k_{1}, k_{2}\right) I_{2, i i}^{1}\left(k_{3}, k_{4}\right)+2 \text { Perm. }
\end{aligned}
$$

The permutations involve the three other choices of $k_{i}$ for the $I_{1, i}^{1}$ term in the first equation, and the two other pairings of the $k_{i}$ 's for the $I_{2, i i}^{1}$ terms in the second. Here we have defined $\mathbf{k}_{12}=\mathbf{k}_{1}+\mathbf{k}_{2}$; note that $k_{12}$ is the length of one of the diagonals in the configuration.

The term containing three halos can only arise with two points in one halo and one in each of the others,

$$
\begin{aligned}
T_{i}^{3 h}= & B^{\operatorname{lin}}\left(\mathbf{k}_{1}, \mathbf{k}_{2}, \mathbf{k}_{34}\right) I_{2, i i}^{1}\left(k_{3}, k_{4}\right) I_{1, i}^{1}\left(k_{1}\right) I_{1, i}^{1}\left(k_{2}\right) \\
& +P^{\operatorname{lin}}\left(k_{1}\right) P^{\operatorname{lin}}\left(k_{2}\right) I_{2, i i}^{2}\left(k_{3}, k_{4}\right) I_{1, i}^{1}\left(k_{1}\right) I_{1, i}^{1}\left(k_{2}\right) \\
& +5 \text { Perm., }
\end{aligned}
$$

where the permutations represent the unique pairings of $k_{i}$ 's in the $I_{2, i i}$ factors. This term also depends on the configuration.

Finally, for four halos, the contribution is

$$
\begin{aligned}
& T_{i}^{4 h}=I_{1, i}^{1}\left(k_{1}\right) I_{1, i}^{1}\left(k_{2}\right) I_{1, i}^{1}\left(k_{3}\right) I_{1, i}^{1}\left(k_{4}\right)\left\{T^{\operatorname{lin}}+\left[\frac{I_{2, i i}^{2}\left(k_{4}\right)}{I_{1, i}^{1}\left(k_{4}\right)}\right.\right. \\
& \left.\left.\times P^{\operatorname{lin}}\left(k_{1}\right) P^{\operatorname{lin}}\left(k_{2}\right) P^{\operatorname{lin}}\left(k_{3}\right)+3 \text { Perm. }\right]\right\} \text {, }
\end{aligned}
$$

where the permutations represent the choice of $k_{i}$ in the $I_{1, i}^{1}$ 's in the brackets. Because of the closure condition expressed by the delta function, the trispectrum may be viewed as a four-sided figure with sides $\mathbf{k}_{i}$. It can alternately be described by the length of the four sides $k_{i}$ plus the diagonals. We occasionally refer to elements of the trispectrum that differ by the length of the diagonals as different configurations of the trispectrum. In the rest of the paper, we will

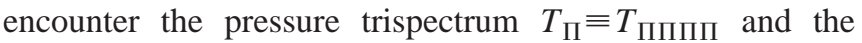
pressure-baryon cross trispectrum $T_{g \Pi g \Pi}$.

\section{PRESSURE TRISPECTRUM AND POWER SPECTRUM COVARIANCE}

Following Ref. [17], we can relate the trispectrum to the variance of the estimator of the binned power spectrum

$$
\hat{P}_{i}=\frac{1}{V} \int_{\mathrm{s} i} \frac{d^{3} k}{V_{\mathrm{s} i}} \delta_{\Pi}^{*}(-\mathbf{k}) \delta_{\Pi}(\mathbf{k}),
$$

where the integral is over a shell in $k$-space centered around $k_{i}, V_{\mathrm{s} i} \approx 4 \pi k_{i}^{2} \delta k$ is the volume of the shell, and $V$ is the volume of the survey. Recalling that $\delta(\mathbf{0}) \rightarrow V /(2 \pi)^{3}$ for a finite volume,

$$
\begin{aligned}
C_{i j} & \equiv\left\langle\hat{P}_{i} \hat{P}_{j}\right\rangle-\left\langle\hat{P}_{i}\right\rangle\left\langle\hat{P}_{j}\right\rangle \\
& =\frac{1}{V}\left[\frac{(2 \pi)^{3}}{V_{\mathrm{s} i}} 2 P_{i}^{2} \delta_{i j}+T_{i j}^{\Pi}\right],
\end{aligned}
$$

where

$$
T_{i j}^{\Pi} \equiv \int_{\mathrm{s} i} \frac{d^{3} k_{i}}{V_{\mathrm{s} i}} \int_{\mathrm{s} j} \frac{d^{3} k_{j}}{V_{\mathrm{s} j}} T_{\Pi}\left(\mathbf{k}_{i},-\mathbf{k}_{i}, \mathbf{k}_{j},-\mathbf{k}_{j}\right) .
$$

Note that though both terms scale in the same way with the volume of the survey, only the Gaussian piece necessarily decreases with the volume of the shell. For the Gaussian piece, the sampling error reduces to a simple root- $N$ mode counting of independent modes in a shell. The trispectrum quantifies the non-independence of the modes both within a shell and between shells. Calculating the covariance matrix of the power spectrum estimates reduces to averaging the elements of the trispectrum across configurations in the shell.

\section{Discussion}

In Fig. 2(a), we show the logarithmic power spectrum of pressure and dark matter such that $\Delta^{2}(k)=k^{3} P(k) / 2 \pi^{2}$, with contributions broken down to the $1 h$ and $2 h$ terms today. As shown, the pressure power spectrum depicts an increase in power relative to the dark matter at scales out to few $h$ $\mathrm{Mpc}^{-1}$, and a decrease thereafter. The decrease in power at small scales can be understood through the relative contribution to pressure as a function of the halo mass. In Fig. 3, we break the total dark matter power spectrum (a) and the total pressure power spectrum (b), to a function of mass. As shown, contributions to dark matter come from massive halos at large scales and by small mass halos at small scales. The pressure power spectrum is such that through temperature weighing, with $T_{e} \propto M^{2 / 3}$ dependence, the contribution from low mass halos to pressure is suppressed relative to that from the high mass end. Thus the pressure power spectrum, at all scales of interest, can be described with halos of mass greater than $10^{14} \mathrm{M}_{\odot}$. A comparison of the dark matter and pressure power spectra, as a function of mass, in Fig. 3 reveals that the turnover in the pressure power spectrum results at an effective scale radius for halos with mass greater than $10^{14} \mathrm{M}_{\odot}$. As we find later, this effective turnover produces a distinct signature in the SZ angular power spectrum inolving a turnover of SZ power at multipoles of few thousand. The presence of this turnover and the exact multipole range can be used as a probe of gas and temperature physics.

For the covariance of pressure, and also for the covariance of the SZ power spectrum, we are mainly interested in terms of the pressure trispectrum involving configurations that result in $T_{\Pi}\left(\mathbf{k}_{1},-\mathbf{k}_{1}, \mathbf{k}_{2},-\mathbf{k}_{2}\right)$, i.e., parallelograms which are defined by either the length $k_{12}$ or the angle between $\mathbf{k}_{1}$ and $\mathbf{k}_{2}$. These are the configurations that contribute to the power spectrum covariance. For illustration purposes we will take $k_{1}=k_{2}$ and the angle to be $90^{\circ}\left(\mathbf{k}_{2}=\mathbf{k}_{\perp}\right)$ such that the parallelogram is a square. It is then convenient to define 

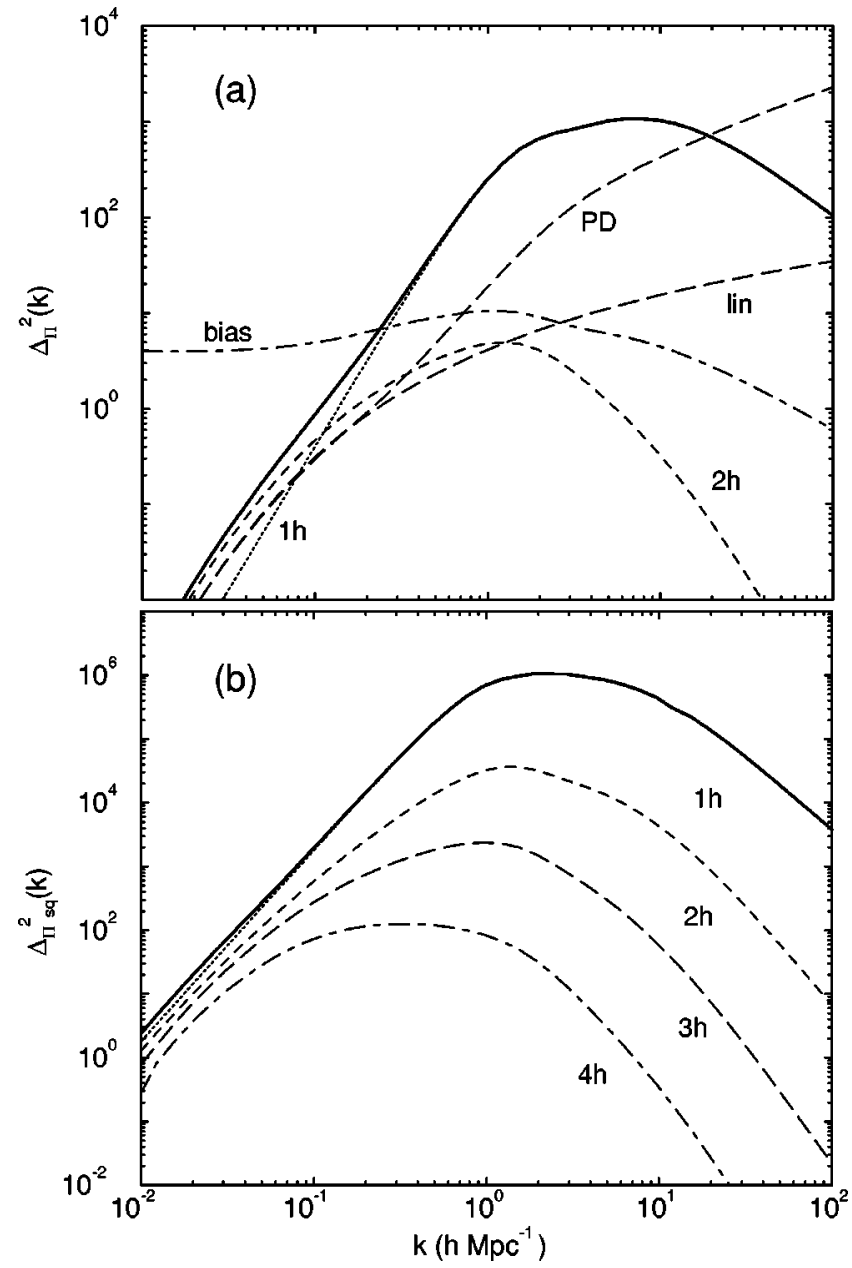

FIG. 2. The pressure power spectrum (a) and squareconfiguration trispectrum $(b)(z=0)$ broken into individual contributions under the halo description. The line labeled "bias" shows the pressure bias relative to the dark matter power spectrum under the halo model. In (b), we show the square configuration trispectrum (see the text). In both (a) and (b), at all scales relevant for the Sunyaev-Zel'dovich effect, the single halo term dominates.

$$
\Delta_{\Pi \text { sq }}^{2}(k) \equiv \frac{k^{3}}{2 \pi^{2}} T_{\Pi}^{1 / 3}\left(\mathbf{k},-\mathbf{k}, \mathbf{k}_{\perp},-\mathbf{k}_{\perp}\right),
$$

such that this quantity scales roughly as the logarithmic power spectrum itself $\Delta^{2}(k)$. This spectrum is shown in Fig. 2(b) with the individual contributions from the $1 h, 2 h, 3 h$, and $4 h$ terms shown. As shown, almost all contributions to the pressure trispectrum come from the single halo term.

Using the pressure trispectrum, we can now predict the pressure covariance and, more appropriately, correlations in the binned measurements of the pressure. The predictions made here with the halo model to describe pressure can easily be tested in numerical simulations, and the accuracy of the halo model can be further studied. For this purpose, we calculate the covariance matrix $C_{i j}$ from Eq. (50) with the bins centered at $k_{i}$ and volume $V_{\mathrm{s} i}=4 \pi k_{i}^{2} \delta k_{i}$. The binning scheme used here is the one we utilized in Ref. [18] to calculate the binned dark matter power spectrum correlations.

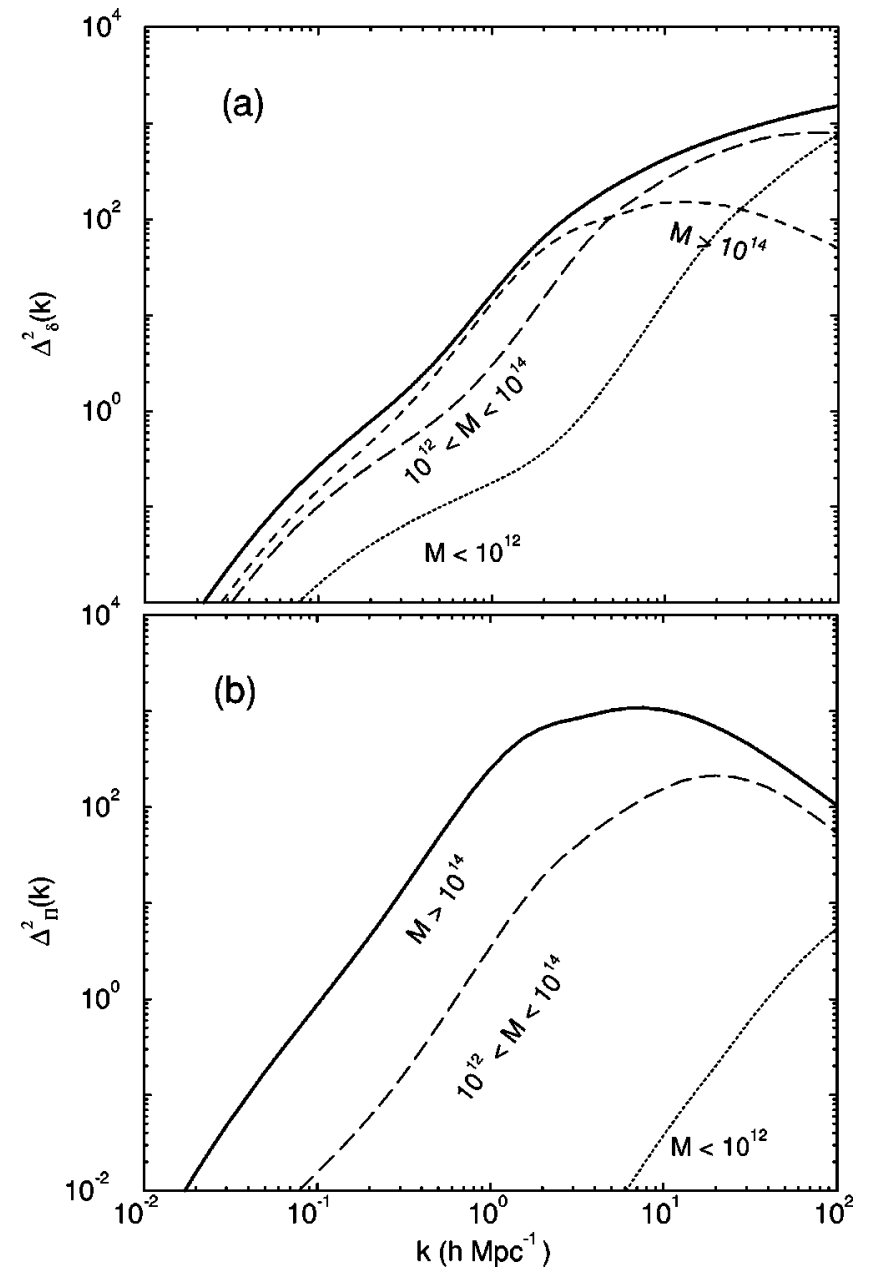

FIG. 3. The mass dependence on the dark matter power spectrum (a) and the pressure power spectrum (b). Here we show the total contribution broken in mass limits as written in the figure. As shown in (a), the large scale contribution to the dark matter power comes from massive halos while small mass halos contribute at small scales. For the pressure, in (b), only massive halos above a mass of $10^{14} \mathrm{M}_{\odot}$ contribute to the power.

In Table I, we tabulate the pressure (upper triangle), and for comparison dark matter (lower triangle), correlation coefficients:

$$
\hat{C}_{i j}=\frac{C_{i j}}{\sqrt{C_{i i} C_{j j}}} .
$$

The dark matter correlations are from the halo based predictions by Ref. [18]. There, for the dark matter, we suggested that the halo model predicted correlations agree with numerical simulations of Ref. [17] typically better than \pm 0.1 , even in the region where non-Gaussian effects dominate, and that the the qualitative features such as the increase in correlations across the nonlinear scale are preserved. As we do not have measurements of the pressure correlations from simulations, we cannot perform a detailed comparison on the accuracy of the halo model predictions for pressure here. 
TABLE I. Pressure power spectrum correlations. Diagonal normalized covariance matrix of the binned pressure (upper triangle) and dark matter density field (lower triangle) power spectrum with $k$ values in units of $h \mathrm{Mpc}^{-1}$. The lower triangle (parenthetical numbers) displays the covariance from halo model in Ref. [18]. The final line shows the fractional increase in the errors (root diagonal covariance) due to non-Gaussianity as calculated under the halo model for pressure and dark matter density field.

\begin{tabular}{|c|c|c|c|c|c|c|c|c|c|c|c|c|}
\hline$k$ & 0.031 & 0.044 & 0.058 & 0.074 & 0.093 & 0.110 & 0.138 & 0.169 & 0.206 & 0.254 & 0.313 & 0.385 \\
\hline 0.031 & 1.000 & 0.243 & 0.337 & 0.386 & 0.398 & 0.396 & 0.385 & 0.371 & 0.359 & 0.350 & 0.343 & 0.337 \\
\hline 0.044 & $(0.019)$ & 1.000 & 0.442 & 0.546 & 0.576 & 0.578 & 0.566 & 0.547 & 0.533 & 0.521 & 0.513 & 0.507 \\
\hline 0.058 & $(0.041)$ & $(0.036)$ & 1.000 & 0.653 & 0.728 & 0.745 & 0.736 & 0.715 & 0.699 & 0.687 & 0.678 & 0.672 \\
\hline 0.074 & $(0.065)$ & $(0.075)$ & $(0.062)$ & 1.000 & 0.807 & 0.865 & 0.868 & 0.847 & 0.832 & 0.819 & 0.811 & 0.805 \\
\hline 0.093 & $(0.086)$ & $(0.111)$ & $(0.118)$ & $(0.102)$ & 1.000 & 0.898 & 0.932 & 0.918 & 0.902 & 0.890 & 0.882 & 0.876 \\
\hline 0.110 & $(0.113)$ & $(0.153)$ & $(0.183)$ & $(0.189)$ & $(0.160)$ & 1.000 & 0.955 & 0.959 & 0.946 & 0.934 & 0.926 & 0.921 \\
\hline 0.138 & (0.149) & (0.204) & $(0.255)$ & $(0.299)$ & $(0.295)$ & $(0.277)$ & 1.000 & 0.978 & 0.973 & 0.961 & 0.953 & 0.947 \\
\hline 0.169 & $(0.172)$ & $(0.238)$ & $(0.302)$ & $(0.368)$ & $(0.368)$ & $(0.433)$ & $(0.434)$ & 1.000 & 0.982 & 0.972 & 0.962 & 0.956 \\
\hline 0.206 & $(0.186)$ & $(0.261)$ & $(0.334)$ & $(0.334)$ & $(0.412)$ & $(0.541)$ & $(0.580)$ & $(0.592)$ & 1.000 & 0.985 & 0.973 & 0.965 \\
\hline 0.254 & $(0.186)$ & $(0.264)$ & $(0.341)$ & $(0.341)$ & $(0.425)$ & $(0.576)$ & $(0.693)$ & $(0.737)$ & $(0.748)$ & 1.000 & 0.986 & 0.973 \\
\hline 0.313 & $(0.172)$ & $(0.251)$ & $(0.328)$ & $(0.328)$ & $(0.412)$ & $(0.570)$ & $(0.698)$ & $(0.778)$ & $(0.839)$ & $(0.858)$ & 1.000 & 0.986 \\
\hline 0.385 & $(0.155)$ & $(0.230)$ & $(0.305)$ & $(0.305)$ & $(0.389)$ & $(0.549)$ & $(0.680)$ & $(0.766)$ & $(0.848)$ & $(0.896)$ & $(0.914)$ & 1.000 \\
\hline$\sqrt{\frac{C_{i i}}{C_{i i}^{G_{\Pi}}}}$ & 1.04 & 1.13 & 1.29 & 1.64 & 1.95 & 2.24 & 3.58 & 6.09 & 9.27 & 16.4 & 21.2 & 29.8 \\
\hline$\sqrt{\frac{C_{i i}}{C_{i i}^{G_{\delta}}}}$ & 1.00 & 1.01 & 1.02 & 1.03 & 1.04 & 1.07 & 1.14 & 1.23 & 1.38 & 1.61 & 1.90 & 2.26 \\
\hline
\end{tabular}

A further test on the accuracy of the halo approach is to consider higher order real-space moments such as the skewness and kurtosis. In Ref. [16], we discussed the SZ skewness under the halo model. As discussed in detail in Ref. [15], halo model predictions agree remarkably well with numerical simulations, especially for the pressure and SZ power spectra, though detailed comparisons still remain to be made with respect to bispectrum and trispectrum.

Even though the dark matter halo formalism provides a physically motivated means of calculating the statistics of the dark matter density field and associated properties such as pressure, there are several limitations of the approach that should be borne in mind when interpreting results. The approach assumes all halos share a parametrized sphericallysymmetric profile and this assumption is likely to affect detailed results on the configuration dependence of the bispectrum and trispectrum. Since we are considering a weighted average of configurations, our predictions presented here may be insufficient to establish the validity of the trispectrum modeling in general. Further numerical work is required to quantify to what extent the present approach reproduces simulation results for the full trispectrum. We do not consider such comparisons here, other than to suggest that the halo model has provided, at least qualitatively, a consistent description better than any of the arguments involving a biased description of gas tracing the dark matter, etc.

\section{SZ THERMAL EFFECT}

The Sunyaev-Zel'dovich [7] effect arises from the inverse Compton scattering of $\mathrm{CMB}$ photons by hot electrons along the line of sight. The temperature decrement along the line of sight due to the SZ effect can be written as the integral of pressure along the same line of sight,

$$
y \equiv \frac{\Delta T}{T_{\mathrm{CMB}}}=g(x) \int d r a(r) \frac{k_{B} \sigma_{T}}{m_{e} c^{2}} n_{e}(r) T_{e}(r),
$$

where $\sigma_{T}$ is the Thomson cross section, $n_{e}$ is the electron number density, $r$ is the comoving distance, and $g(x)$ $=x \operatorname{coth}(x / 2)-4$ with $x=h \nu / k_{B} T_{\mathrm{CMB}}$ is the spectral shape of the SZ effect. At the Rayleigh-Jeans part of the CMB, $g(x)$ $=-2$. For the rest of this paper, we assume observations in the Rayleigh-Jeans regime of the spectrum; an experiment such as Planck's with a sensitivity beyond the peak of the spectrum can separate out SZ contributions based on the spectral signature, $g(x)[10]$.

\section{A. Power spectrum and trispectrum}

The SZ power spectrum and trispectrum are defined in the flat sky approximation in the usual way:

$$
\left\langle y\left(\mathbf{l}_{1}\right) y\left(\mathbf{l}_{2}\right)\right\rangle=(2 \pi)^{2} \delta_{\mathrm{D}}\left(\mathbf{l}_{12}\right) C_{l}^{\mathrm{SZ}},
$$

$$
\left\langle y\left(\mathbf{l}_{1}\right) \ldots y\left(\mathbf{l}_{4}\right)\right\rangle_{c}=(2 \pi)^{2} \delta_{\mathrm{D}}\left(\mathbf{l}_{1234}\right) T^{\mathrm{SZ}}\left(\mathbf{l}_{1}, \mathbf{l}_{2}, \mathbf{l}_{3}, \mathbf{l}_{4}\right) .
$$

These can be written as a redshift projection of the pressure power spectrum, 


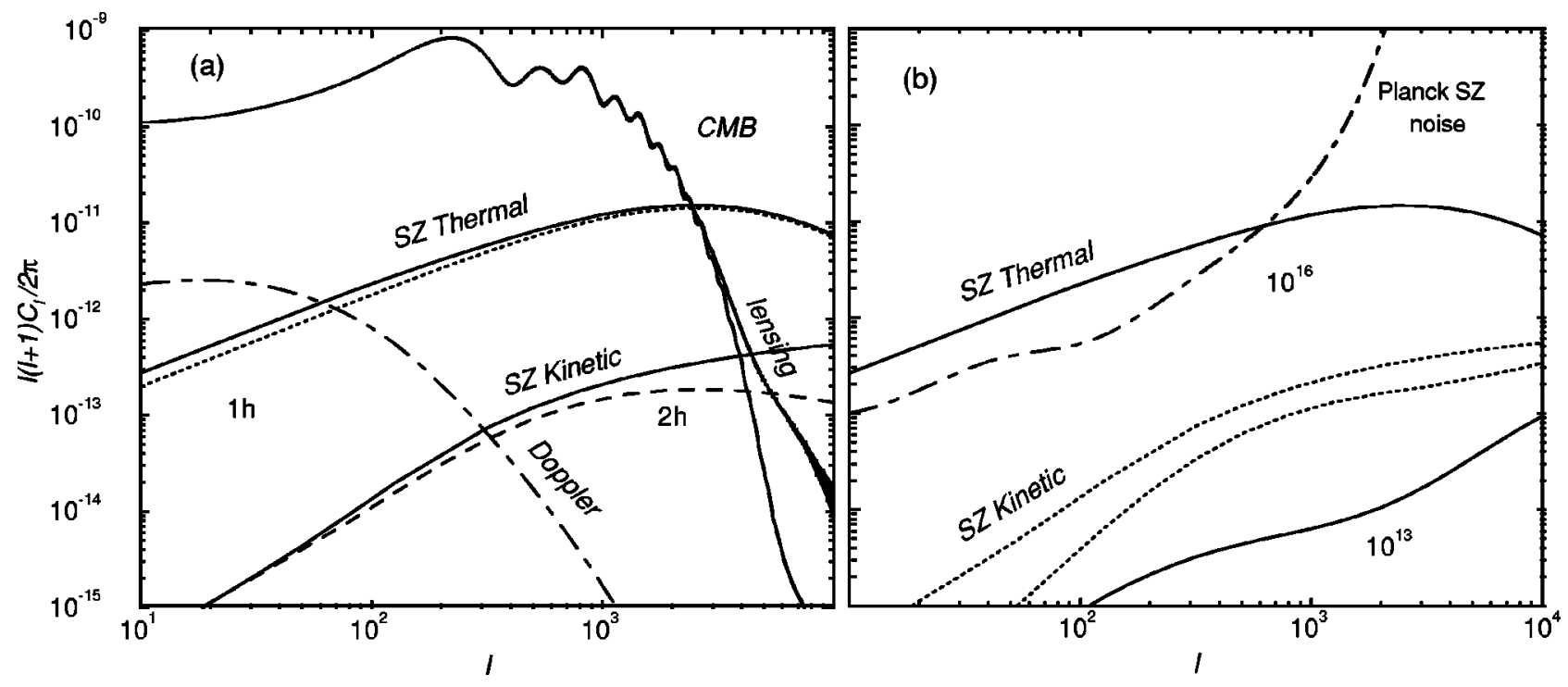

FIG. 4. The angular power spectra of SZ thermal and kinetic effects. As shown in (a), the thermal SZ effect is dominated by individual halos, and thus by the single halo term, while the kinetic effect is dominated by the large scale structure correlations depicted by the two-halo term. In (b), we show the mass dependence of the SZ thermal and kinetic effects with a maximum mass of $10^{16}$ and $10^{13} \mathrm{M}_{\odot}$. The $\mathrm{SZ}$ thermal effect is strongly dependent on the maximum mass while, due to large scale correlations, the kinetic effect is not.

$C_{l}^{\mathrm{SZ}}=\int d r \frac{W^{\mathrm{SZ}}(r)^{2}}{d_{A}^{2}} P_{\Pi}^{\mathrm{t}}\left(\frac{l}{d_{A}}, r\right)$,

$T^{\mathrm{SZ}}=\int d r \frac{W^{\mathrm{SZ}}(r)^{4}}{d_{A}^{6}} T_{\Pi}\left(\frac{\mathbf{l}_{1}}{d_{A}}, \frac{\mathbf{l}_{2}}{d_{A}}, \frac{\mathbf{l}_{3}}{d_{A}}, \frac{\mathbf{l}_{4}}{d_{A}}, ; r\right)$,

where $d_{A}$ is the angular diameter distance. At the RJ part of the frequency spectrum, the SZ weight function is

$$
W^{\mathrm{SZ}}(r)=-2 \frac{k_{B} \sigma_{T} \bar{n}_{e}}{a(r)^{2} m_{e} c^{2}}
$$

where $\bar{n}_{e}$ is the mean electron density today. In deriving Eq. (56), we have used the Limber approximation [37], by setting $k=l / d_{A}$, and the flat-sky approximation.

In Fig. 4(a), we show the SZ power spectrum due to baryons present in virialized halos. As shown, most of the contributions to the SZ power spectrum comes from individual massive halos, while the halo-halo correlations only contribute at a level of $10 \%$ at large angular scales. This is contrary to, say, the lensing convergence power spectrum discussed in Ref. [29], where most of the power at large angular scales is due to the halo-halo correlations. The difference can be understood by noting that the SZ effect is strongly sensitive to the most massive halos due to the $T \propto M^{2 / 3}$ dependence on temperature and to a lesser, but somewhat related, extent that the weight function increases toward low redshifts. The lensing weight function selectively probes the large scale dark matter density power spectrum at comoving distances half to that of background sources $(z \sim 0.2$ to 0.5 when sources are at a redshift of 1), but has no extra dependence on mass. The fact that the SZ power spectrum results mainly from the single halo term also results in a sharp reduction of power when the maximum mass used in the calculation is varied.
For example, as discussed in Ref. [16] and illustrated in Fig 4(b), with the maximum mass decreased from $10^{16}$ to $10^{13} \mathrm{M}_{\odot}$, the SZ power spectrum is reduced by a factor of nearly two orders of magnitude in large scales and an order of magnitude at $l \sim 10^{4}$. In Fig. 5, we show the scaled trispectrum

$$
\Delta_{\mathrm{sq}}^{\mathrm{SZ}}(l)=\frac{l^{2}}{2 \pi} T^{\mathrm{SZ}}\left(\mathbf{l},-\mathbf{l}, \mathbf{l}_{\perp},-\mathbf{l}_{\perp}\right)^{1 / 3},
$$

where $l_{\perp}=l$ and $\mathbf{l} \cdot \mathbf{l}_{\perp}=0$. The projected SZ trispectrum again shows the same behavior as the pressure trispectrum. As

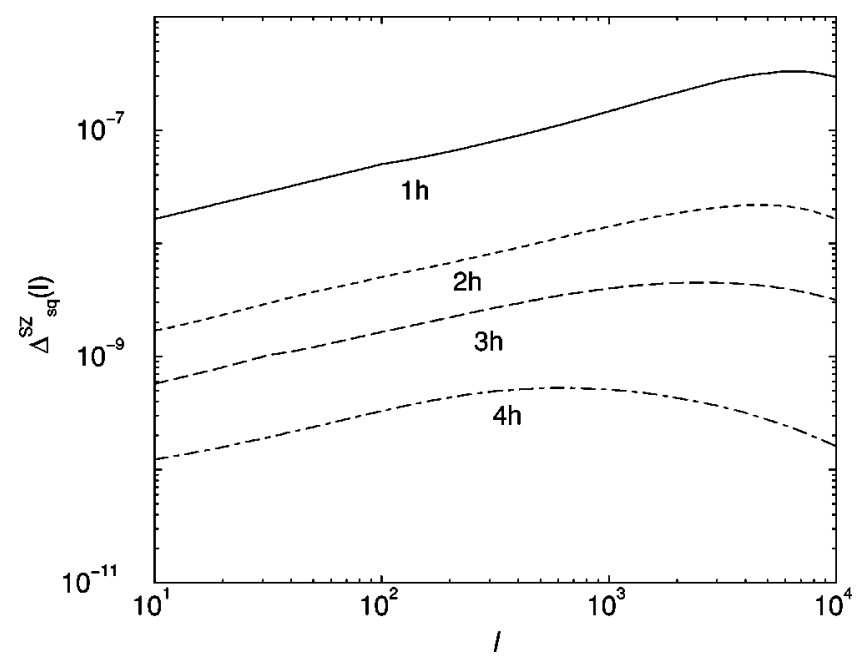

FIG. 5. SZ trispectrum for a configuration defined by a square under the halo model. As shown, the single halo term dominates the contribution to the trispectrum at all multipoles ranging from large angular scales to small angular scales. The dependence on the single halo term is consistent with the general non-Gaussian behavior of the SZ effect and its significant nonlinearity. 


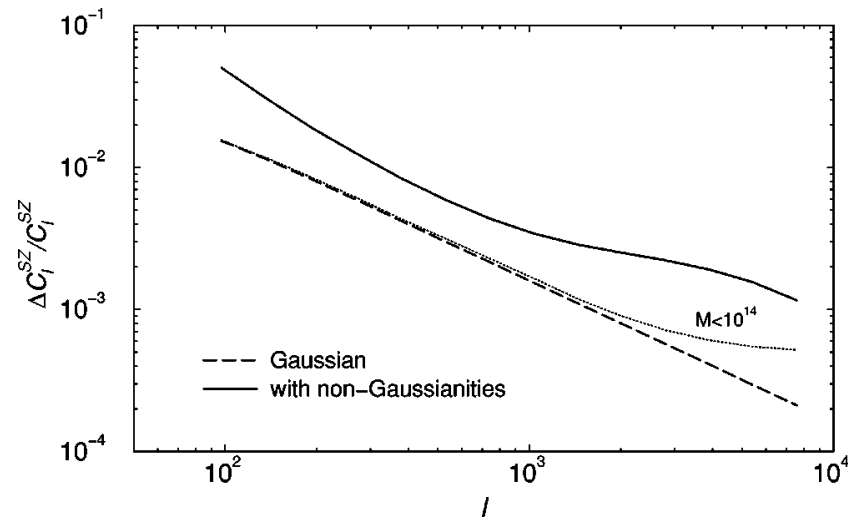

FIG. 6. The fractional errors in the measurements of the SZ band powers. Here we show the fractional errors under the Gaussian approximation, and the total including non-Gaussianities. As shown, the total contribution as a function of mass is sensitive to the presence of most massive halos in the universe. The nonGaussian term is essentially dominated by the single halo term.

shown, contributions to the trispectrum essentially come from the single halo term at all multipoles. This is consistent with our observation that the SZ power spectrum is essentially dominated by the correlations of pressure within halos. As discussed in Ref. [16], the SZ bispectrum is also dominated by the single halo term. Given this dependence on the single halo term, for the rest of the discussion involving SZ covariance we will only use the single halo term, and ignore the contributions arising from large scale correlations associated with halos.

\section{B. SZ power spectrum covariance}

For the purpose of this calculation, we assume that planned small angular scale SZ experiments will measure the thermal SZ power spectrum in bins of logarithmic band powers at several $l_{i}$ 's in multipole space with thickness $\delta l_{i}$. Thus we can write the band power measurements as

$$
\mathcal{C}_{i}=\int_{\mathrm{s} i} \frac{d^{2} l}{A_{\mathrm{s} i}} \frac{l^{2}}{2 \pi} y(\mathbf{l}) y(-\mathbf{l}),
$$

where $A_{\mathrm{s}}\left(l_{i}\right)=\int d^{2} l$ is the area of two-dimensional shell in the multipole, and can be written as $A_{\mathrm{s}}\left(l_{i}\right)=2 \pi l_{i} \delta l_{i}$ $+\pi\left(\delta l_{i}\right)^{2}$.

We can now write the signal covariance matrix as

$C_{i j}=\frac{1}{A}\left[\frac{(2 \pi)^{2}}{A_{\mathrm{s} i}} 2 \mathcal{C}_{i}^{2}+T_{i j}^{\mathrm{SZ}}\right]$

$T_{i j}^{\mathrm{SZ}}=\int \frac{d^{2} l_{i}}{A_{\mathrm{si}}} \int \frac{d^{2} l_{j}}{A_{\mathrm{s} j}} \frac{l_{i}^{2} l_{j}^{2}}{(2 \pi)^{2}} T^{\mathrm{SZ}}\left(\mathbf{l}_{i},-\mathbf{l}_{i}, \mathbf{l}_{j},-\mathbf{l}_{j}\right)$,

where $A$ is the area of the survey in steradians. Again the first term is the Gaussian contribution to the sample variance, and the second term is the non-Gaussian contribution. A realistic survey will also have an additional noise variance due to the instrumental effects and a covariance resulting from the un- certainties associated with the separation of the SZ effect from thermal CMB and other foregrounds.

In Fig. 6, we show the fractional error

$$
\frac{\Delta \mathcal{C}_{i}}{\mathcal{C}_{i}} \equiv \frac{\sqrt{C_{i i}}}{\mathcal{C}_{i}}
$$

for bands $l_{i}$ given in Table II following the binning scheme used in Ref. [18] for the weak lensing power spectrum.

In Fig. 6, the dashed line shows the Gaussian error while the solid line shows the total covariance with the addition of the SZ trispectrum [Eq. (60)]. At all multipoles, the nonGaussianities from the trispectrum dominate the variance. As we discussed for the power spectrum, however, a reduction in the maximum mass of the halos used for the SZ calculation leads to a sharp decreases in the non-Gaussianities. With a mass cut at $10^{14} \mathrm{M}_{\odot}$, shown by the dotted line, we see that the total variance is consistent with the Gaussian variance out to $l \sim 1000$.

We can now write the correlation between the bands as

$$
\hat{C}_{i j} \equiv \frac{C_{i j}}{\sqrt{C_{i i} C_{j j}}} .
$$

In Table II we tabulate the SZ correlations under the assumption that the SZ power spectrum is measured independently, say in a frequency cleaned map (upper triangle), and is measured in the CMB primary dominated map (lower triangle). The correlations along individual columns increase (as one goes to large $l$ 's or small angular scales) and the maximum values are reached at $l \sim 5000$ consistent with the general behavior of the trispectrum.

In Fig. 7, we show the non-Gaussian trispectrum correlation coefficient given by

$$
\hat{C}_{i j}^{\mathrm{NG}}=\frac{T_{i j}}{\sqrt{T_{i i} T_{i j}}} .
$$

As shown here, the increase in non-Gaussian correlation is consistent with the fact that at all scales it is the single halo term which dominates the non-Gaussian contribution. Since the power spectrum is dominated by correlations in single halos, the fixed profile of the halos correlate the power in all the modes and the correlations between adjacent modes are significant.

The calculation, or experimental measurement, of the full SZ covariance is necessary for an interpretation of observational results on the power spectrum. The upcoming SZ surveys, where the power spectrum will be measured, is likely to be limited to a small area on the sky. Thus in the absence of many fields where the covariance can be estimated directly from the data, the halo model based approach suggested here provides a useful, albeit model dependent, quantification of the covariance. As suggested for weak lensing observations in Ref. [38] and discussed in Ref. [18], as a practical approach one could imagine taking the variances estimated from the survey under a Gaussian approximation, after accounting for uneven sampling and edge effects, and scaling it up by the non-Gaussian to Gaussian variance ratio 
TABLE II. SZ thermal power spectrum correlations. Covariance of the binned power spectrum for the SZ effect. The upper triangle displays the covariance found when a perfect frequency cleaned SZ map is used to determine the SZ power spectrum. The lower triangle (parenthetical numbers) displays the covariance found when the variance is dominated by the primary anisotropy contribution, as in a measurement of the SZ power spectrum in a CMB primary fluctuations dominated map.

\begin{tabular}{lcccccccccccccc}
\hline \hline$l_{\text {bin }}$ & 97 & 138 & 194 & 271 & 378 & 529 & 739 & 1031 & 1440 & 2012 & 2802 & 3905 & 5432 & 7568 \\
\hline 97 & 1.00 & 0.90 & 0.71 & 0.49 & 0.30 & 0.18 & 0.09 & 0.05 & 0.02 & 0.01 & 0.00 & 0.00 & 0.00 & 0.00 \\
138 & $(0.00)$ & 1.00 & 0.90 & 0.70 & 0.49 & 0.30 & 0.17 & 0.09 & 0.05 & 0.03 & 0.02 & 0.02 & 0.01 & 0.00 \\
194 & $(0.00)$ & $(0.00)$ & 1.00 & 0.89 & 0.70 & 0.48 & 0.30 & 0.17 & 0.09 & 0.05 & 0.03 & 0.03 & 0.01 & 0.00 \\
271 & $(0.00)$ & $(0.00)$ & $(0.00)$ & 1.00 & 0.89 & 0.69 & 0.47 & 0.30 & 0.17 & 0.09 & 0.05 & 0.02 & 0.01 & 0.00 \\
378 & $(0.00)$ & $(0.00)$ & $(0.00)$ & $(0.00)$ & 1.00 & 0.89 & 0.69 & 0.49 & 0.30 & 0.17 & 0.09 & 0.05 & 0.03 & 0.01 \\
529 & $(0.00)$ & $(0.00)$ & $(0.00)$ & $(0.00)$ & $(0.00)$ & 1.00 & 0.88 & 0.69 & 0.48 & 0.30 & 0.17 & 0.17 & 0.11 & 0.07 \\
739 & $(0.00)$ & $(0.00)$ & $(0.00)$ & $(0.00)$ & $(0.00)$ & $(0.00)$ & 1.00 & 0.88 & 0.68 & 0.49 & 0.38 & 0.28 & 0.20 & 0.13 \\
1031 & $(0.00)$ & $(0.00)$ & $(0.00)$ & $(0.00)$ & $(0.00)$ & $(0.00)$ & $(0.00)$ & 1.00 & 0.87 & 0.68 & 0.56 & 0.43 & 0.31 & 0.21 \\
1440 & $(0.00)$ & $(0.00)$ & $(0.00)$ & $(0.00)$ & $(0.00)$ & $(0.00)$ & $(0.00)$ & $(0.00)$ & 1.00 & 0.88 & 0.69 & 0.61 & 0.45 & 0.31 \\
2012 & $(0.00)$ & $(0.00)$ & $(0.00)$ & $(0.00)$ & $(0.00)$ & $(0.00)$ & $(0.00)$ & $(0.01)$ & $(0.05)$ & 1.00 & 0.88 & 0.69 & 0.60 & 0.42 \\
2802 & $(0.00)$ & $(0.00)$ & $(0.00)$ & $(0.00)$ & $(0.00)$ & $(0.00)$ & $(0.00)$ & $(0.02)$ & $(0.09)$ & $(0.39)$ & 1.00 & 0.88 & 0.70 & 0.56 \\
3905 & $(0.00)$ & $(0.00)$ & $(0.00)$ & $(0.00)$ & $(0.00)$ & $(0.00)$ & $(0.00)$ & $(0.02)$ & $(0.08)$ & $(0.36)$ & $(0.84)$ & 1.00 & 0.87 & 0.70 \\
5432 & $(0.00)$ & $(0.00)$ & $(0.00)$ & $(0.00)$ & $(0.00)$ & $(0.00)$ & $(0.00)$ & $(0.01)$ & $(0.06)$ & $(0.29)$ & $(0.65)$ & $(0.86)$ & 1.00 & 0.88 \\
7568 & $(0.00)$ & $(0.00)$ & $(0.00)$ & $(0.00)$ & $(0.00)$ & $(0.00)$ & $(0.00)$ & $(0.01)$ & $(0.04)$ & $(0.20)$ & $(0.53)$ & $(0.70)$ & $(0.88)$ & 1.00 \\
\hline \hline
\end{tabular}

of the halo model along with inclusion of the band power correlations. Additionally, using a covariance like the one calculated here, one can use the approach well known in the fields of CMB and galaxy power spectrum measurements to decorrelate band powers [39].

\section{Astrophysical uses of the SZ power spectrum}

The calculation of the full covariance matrix now allows us to study how well the SZ power spectrum measures certain astrophysical and cosmological parameters. The upcoming $\mathrm{CMB}$ power spectrum measurements, complemented by

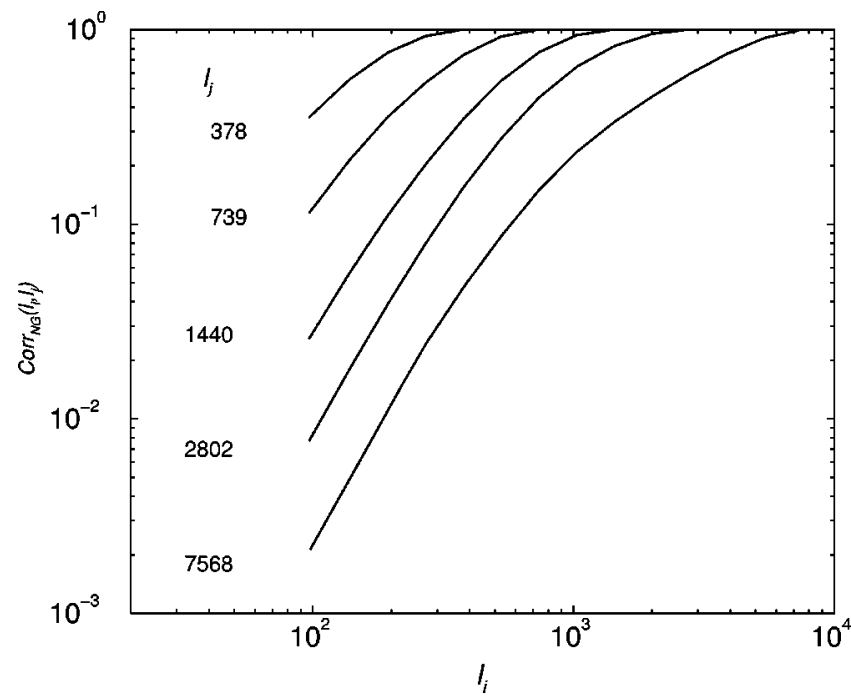

FIG. 7. The non-Gaussian correlation coefficient $\hat{C}_{i j}^{\mathrm{NG}}$, of the SZ power spectrum, involving only the configuration of the SZ trispectrum that contributes to the SZ power spectrum covariance [see Eq. (63)]. The correlations are such that they tend to 1 as $l_{i} \rightarrow l_{j}$, and are fully described by the contribution to the trispectrum by the single halo term. the related local universe observations such as the galaxy power spectrum or supernovas, are expected to constrain most of the cosmological parameters to a reasonable accuracy [40]. Thus we ignore the possibility that the SZ effect can be used as a probe of cosmology and only concentrate on the astrophysical uses of the SZ effect. This is a reasonable approach to take since there are many unknown astrophysics associated with the SZ effect involving the clustering of gas density and temperature. Such an approach allows us not to complicate the parameter measurements by adding both astrophysical and cosmological parameters. Assuming the cosmology will be safely known, we now ask the question what additional astrophysical parameters one can hope to extract from the SZ effect under the present halo model.

There are many approaches to parametrize the unknown astrophysics of the SZ power spectrum. Some possibilities were already suggested in the literature, essentially involving the gas evolution [41]. Since the SZ effect involves both gas and temperature as a product, i.e. the pressure, one may be led to conclude that it is not possible to separate effects associated with temperature from those associated with gas density. Given the dependence of temperature on the pressure profile, which we calculated from hydrostatic equilibrium, one can expect the degeneracy between gas properties and temperature properties to be partly broken. Note that the profile shape only depends on the temperature and not the exact density of gas; thus the profile shape is only affected by the temperature. This statement is exact under the assumption that viral equation can be applied. If one were to consider a varying temperature distribution, then the pressure profile would be sensitive to both gas density and temperature. We do not consider the case with a temperature profile as we are only interested in the most simplest case to describe pressure here.

As discussed earlier, the clustering of pressure power spectrum has a turnover corresponding to an equivalent scale 


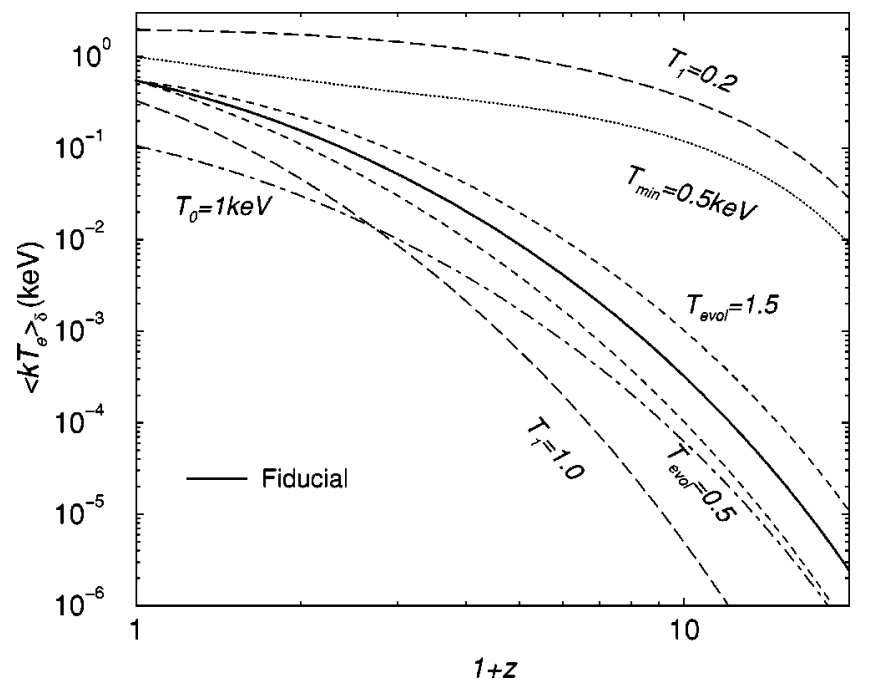

FIG. 8. The variation in the density weighted temperature of electrons as a function of the redshift. The solid line shows the redshift evolution of the temperature under the fiducial model, while variations about this model are shown as labeled.

radius of pressure. Through the gas pressure profile, this turnover can be characterized by the parameter $b$ and the dark matter scale radius $r_{s}$. Note that $b \propto 1 / T_{e}$, so a measurement of $b$ is essentially a probe of the electron temperature, though it is unlikely that one can obtain all information on temperature and its evolution from one parameter measurement. Thus, instead of $b$, we take temperature itself to be one interesting astrophysical parameter and consider its evolution such that

$$
T(M, z)=T_{0}\left(\frac{M}{10^{15} h^{-1} M_{\odot}}\right)^{T_{1}}(1+z)^{T_{\text {evol }}}+T_{\text {min }} .
$$

Here the four parameters represent the temperature-mass normalization $T_{0}$, which in the fiducial case has a value given by the virial equation $\left(K_{B} T_{0} \sim 5.2 \mathrm{keV}\right)$; the mass dependence slope $T_{1}$, with a fiducial value of $2 / 3$; a redshift dependent evolutionary parameter $T_{\text {evol }}$, with a fiducial value of 1; and a minimum temperature for gas independent of mass and redshift $T_{\min }$, with a value of zero in the fiducial case. This latter parameter accounts for any possible preheating of gas before virializing in halos due to effects associated with some unknown astrophysics, such as the reionization process. A measurement of $T_{\min }$ would be interesting given that observational data from massive clusters to less massive galaxy groups suggest possible preheating of gas before virialization in halos. As discussed in Refs. [16] and [14], the SZ power spectrum provides a strong probe of preheating of gas. We note here that our description of preheating as a redshift independent temperature is likely to be too extreme since one expects the preheating temperature to vary with both redshift and mass such that all three parameters, $T_{0}, T_{1}$, and $T_{\text {evol }}$, are affected. We do not consider variations in $T_{\min }$ as a function of redshift due to our unknown knowledge of physics associated with this process and that addition of extra parameters can lead to further degeneracies. In Fig. 8, we show the variation in the redshift evolution of the density weighted temperature of electrons about the fiducial model. The density weight temperature was calculated following Eq. (31).

In addition to the temperature, the SZ effect also depends on the number density of electrons in clusters. So far, we have considered this number through the universal baryon fraction in the universe such that $f_{g} \equiv M_{g} / M_{\delta}=\Omega_{g} / \Omega_{m}$. This assumption ignores any possible effects associated with the evolution of the gas fraction in halos, independent of any evolution that may be associated with temperature. It will be interesting to study to what extent future observations will allow the measurement of the fraction of baryons that is responsible for the SZ effect, and any evolution that may be associated with this fraction. Thus a second set of parameters one can hope to extract from SZ observations involves gas mass fraction of halos and its evolution.

To study such gas properties, we parametrize the gas mass fraction such that

$$
f_{g}=f_{0}\left(\frac{M}{10^{15} h^{-1} M_{\odot}}\right)^{f_{1}}(1+z)^{f_{\text {evol }}} .
$$

In a recent paper, Majumdar [41] suggested the possible measurement of any mass and redshift dependence of gas mass fraction in galaxy clusters, given that the SZ power spectrum was observed to vary significantly with changes in these two parameters. Since the SZ power spectrum essentially is sensitive to $\sim f_{g}^{2} T_{e}^{2}$, however, such a suggestion for a measurement of gas evolution is not independent of any variations associated with temperature, which was ignored in the study of Ref. [41]. Our general parametrization above involving both temperature and gas allows us to quantify how well independent statements can be made on possible measurement of gas density and temperature evolution, under the assumption that cosmology is known. Note that gas evolution is not present in our fiducial model since we take the gas fraction to be independent of mass and redshift with $f_{1}=0$ and $f_{\text {evol }}=0$, respectively.

We now have a total of seven parameters we wish to extract from a measurement of the SZ power spectrum. In order to perform this calculation we take a Fisher matrix based approach. The Fisher matrix is simply a projection of the covariance matrix onto the basis of astrophysical parameters $p_{i}$,

$$
\mathbf{F}_{\alpha \beta}=\sum_{i j} \frac{\partial \mathcal{C}_{i}}{\partial p_{\alpha}}\left(C_{\text {tot }}^{-1}\right)_{i j} \frac{\partial \mathcal{C}_{j}}{\partial p_{\beta}},
$$

where the total covariance includes both the signal and noise covariance. Under the approximation of Gaussian shot noise, this reduces to replacing $C_{l}^{\mathrm{SZ}} \rightarrow C_{l}^{\mathrm{SZ}}+C_{l}^{\text {Noise }}$ in the expressions leading up to the covariance equation (60). The noise power spectrum includes the noise associated with detectors, beam size and variance resulting from the separation of the $\mathrm{SZ}$ effect from other temperature fluctuations in multifrequency data.

Under the approximation that there are a sufficient number of modes in the band powers that the distribution of power spectrum estimates is approximately Gaussian, the 


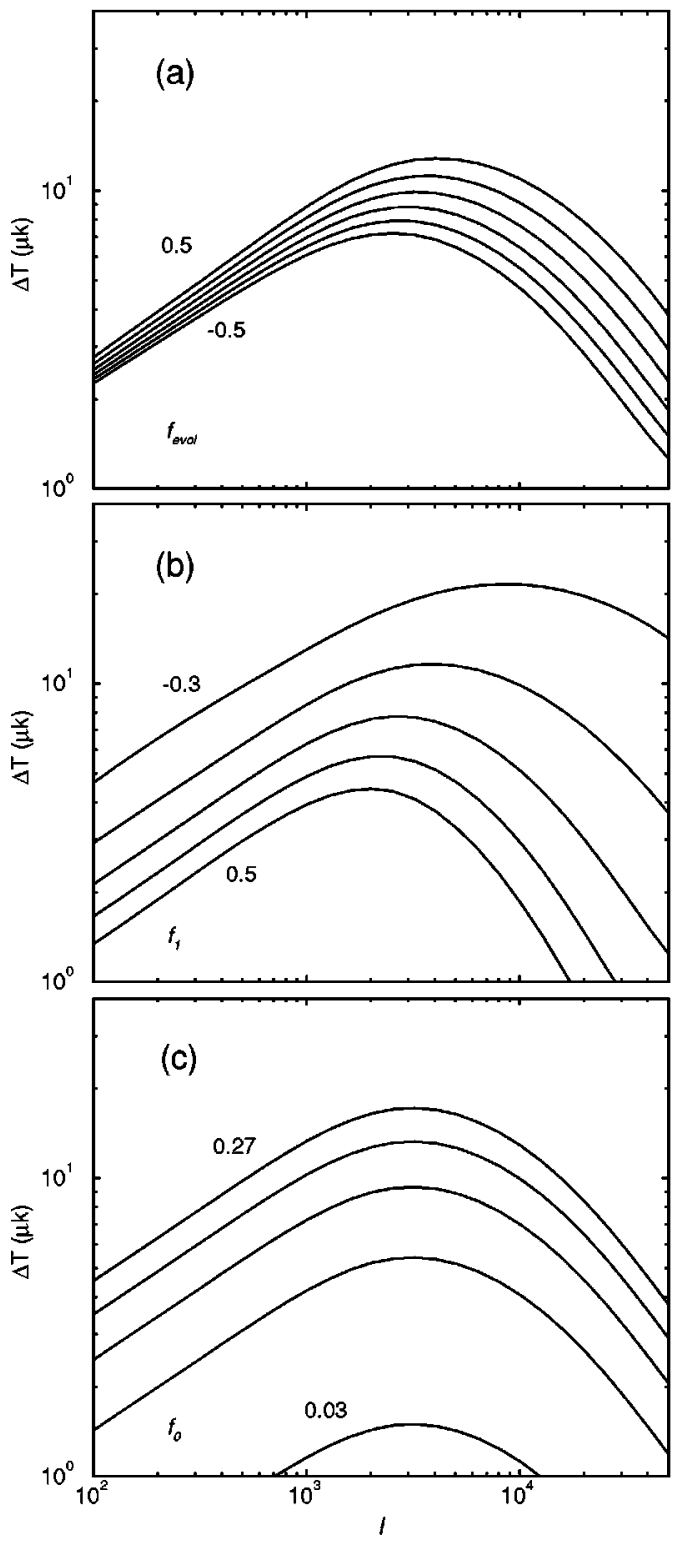

Fisher matrix quantifies the best possible errors on cosmological parameters that can be achieved by a given survey. In particular $F^{-1}$ is the optimal covariance matrix of the parameters and $\left(F^{-1}\right)_{i i}^{1 / 2}$ is the optimal error on the $i$ th parameter. Implicit in this approximation of the Fisher matrix is the neglect of information from the parameter dependence of the covariance matrix of the band powers themselves. We neglect this information due to computational restrictions on the calculation of covariance for all variations in parameters within a reasonable amount of time. We do not expect this exclusion to change our results significantly. Also, here, we are mostly interested in an order of magnitude estimate on how well the SZ power spectrum can constrain astrophysics associated with large scale pressure.

The Fisher matrix approach allows us to address how well degeneracies are broken in the parameter space under the assumption of a fiducial model for the parameters. For the purpose of this calculation, we take binned measurements of the SZ power spectrum following the binning scheme in Table II. We consider a perfect (no instrumental noise), SZ
FIG. 9. The temperature fluctuations of the SZ effect through variations in the astrophysical parameters under the halo model. From (a) to (c), we show the variations associated with gas evolution, while from (d) to (f), we show variations involved with temperature. The parameters are described in Sec. IV C. 
TABLE III. Inverse Fisher matrix $\left(\times 10^{2}\right)$. Inverse Fisher matrix for the SZ effect with seven parameters and full non-Gaussian errors. The error on an individual parameter is the square root of the diagonal element of the inverse Fisher matrix for the parameter, while off-diagonal entries of the inverse Fisher matrix shows correlations, and, thus, degeneracies, between parameters. We have assumed a perfect, no instrumental noise, experiment with a full sky survey $\left(f_{\text {sky }}=1\right)$. The seven parameters are described in Sec. IV C.

\begin{tabular}{lccccccc}
\hline \hline$p_{i}$ & $T_{0}$ & $T_{1}$ & $T_{\text {evol }}$ & $T_{\text {min }}$ & $f_{0}$ & $f_{1}$ & $f_{\text {evol }}$ \\
\hline$T_{0}$ & 8.80 & 1.32 & 3.67 & -1.93 & -0.21 & -1.69 & -3.36 \\
$T_{1}$ & & 0.51 & 1.08 & -0.18 & -0.04 & -0.41 & -0.07 \\
$T_{\text {evol }}$ & & & 2.69 & -0.62 & -0.11 & -0.94 & -2.11 \\
$T_{\text {min }}$ & & & & 0.48 & 0.04 & 0.29 & 0.67 \\
$f_{0}$ & & & & & 0.006 & 0.05 & 0.09 \\
$f_{1}$ & & & & & & 0.39 & 0.73 \\
$f_{\text {evol }}$ & & & & & & & 1.67 \\
\hline \hline
\end{tabular}

profiles of halos. For the most part, variations due to temperature evolution is due to the normalization and not due to variations in the profile shape. In Figs. 9(b) and 9(e), we show variations due to the mass slope of the gas evolution and temperature evolution, respectively. Here again, we see similar behaviors. When the slope of the mass-temperature relation, as a function of mass, is greater than 0.7 , we see significant differences, especially involving an increase in temperature fluctuations at small scales. This is due to the relatively increasing weighing of small mass halos.

In Figs. 9(c) and 9(f), we show variations associated with gas evolution normalization $f_{0}$ and temperature-mass normalization $T_{0}$. The variation associated with $f_{0}$ is easily understood since the effect is only a change in the overall normalization of the power spectrum. The variation with temperature-mass normalization shows both due to effects of normalization and the profile. When the normalization is low, gas clusters to small radii in low mass halos leading to an increase in power at small scales. As the temperature normalization is increased, gas profile varies such that there is a reduction in small scale power and the angular multipole of the turnover scale shifts to low values. When the temperature normalization is sufficiently high, the overall weighing resulting from the overall temperature multiplicative factor becomes important. Now the power spectrum behaves as a simple normalization change, similar to the variation in power due to gas evolution normalization. As shown in Figs. 9(a)-9(f), there are significant degeneracies involved with astrophysical parameters that lead to the SZ effect.

In Table III, we tabulate the errors on these seven parameters using the inverse Fisher matrix for a possible SZ power spectrum measurement. Here we consider the possibility that parameter extraction will be limited to three, five, and seven parameters. The increase in number of parameters to be measured from a SZ power spectrum increases degeneracies associated with the set of parameters resulting in their accuracies. In the case of the three parameters involving temperature-mass normalization, $T_{0}$, a minimum temperature for all halos $T_{\min }$, and the gas mass fraction $f_{0}$, in a perfect experiment all three parameters can be extracted such that they will provide essentially very strong constraints. For example, the error on $f_{0}$ is such that one can identify the gas fraction of clusters responsible for the SZ effect from the cosmic mean of $\Omega_{g} / \Omega_{m}=0.05 / 0.3$ with an error of 4 $\times 10^{-3}$. With the Planck effect one can constrain the preheating temperature at the level of $\sim 0.7 \mathrm{keV}$, and since current predictions for possible preheating is also at the level of few tenths $\mathrm{keV}$, the Planck SZ power spectrum can either confirm or put a useful limit on preheating temperature at current expectations.

As tabulated, however, the accuracy to which parameters can be determined from the SZ power spectrum reduces significantly when the number of parameters to be determined is increased. For example, the Planck mission will only set a limit at $\sim 1.7 \mathrm{keV}$ on preheating, if one were to study both the mass and redshift dependence of electron temperatures. Such an upper limit is unlikely to be useful for current studies related to preheating of gas. Given that we cannot obtain useful errors with the Planck approach for two parameters, we suggest that the Planck approach may not be useful for the purpose of studying the full parameter space suggested here. This is understandable since the Planck approach allows only measurement of the SZ power spectrum out to $l$ $\sim 1500$, while most of the variations due to parameters under discussion here happens at $l \sim 5000$ or higher where the turnover in the SZ power spectrum is observed. The Planck mission, however, allows one to obtain reasonable errors on parameters which generally define the normalization of the power spectrum, such as the temperature-mass normalization or the normalization of gas mass fraction. The normalization for gas mass fraction from the Planck approach will be useful for the purpose of understanding what fraction of cosmic baryons reside in massive halos and contribute to the SZ effect, and to look for any discrepancy of such a value from the total baryon content predicted by big bang nucleosynthesis arguments. In order to obtain reliable measurements of evolution of gas and temperature, a small scale experiment sensitive to multipoles out to $l \sim 10^{4}$ will be necessary.

For a perfect experiment, we also show the errors on seven parameters in Table IV. The inverse Fisher matrix in this case is tabulated in Table III. The diagonals of the inverse Fisher matrix show the variance of individual parameters, while, more importantly, the off diagonals show the covariance between parameters. These covariances allow one to understand the degeneracies between parameters. In Table IV, we show the full extent to which parameters degrade the accuracies by tabulating degradation factors associated with the seven parameters. The degradation factor shows the increase in parameter error from what can be achieved if all other parameters are known to what can be achieved when all parameters are to be retrieved from data. The degradation factors are at the level of 100 or more for some parameters, suggesting that there are significant degeneracies associated with the parametrization of the temperature and gas fraction as a function of mass and redshift. Our results generally suggest that accurate estimates of gas evolutionary properties, in the presence of unknown temperature properties, are not possible. This conclusion, though inconsistent with what was suggested by Majumdar in Ref. [41], we believe that this was 
TABLE IV. Parameter errors. Parameter errors, $\left(F^{-1}\right)_{i i}^{1 / 2}$, using the halo model and the full covariance for the SZ effect. We tabulate these errors for a perfect experiment with no instrumental noise and full sky observations out to $l \sim 10^{4}$. We also show the expected errors for Planck mission with a useful sky fraction of $65 \%\left(f_{\text {sky }}\right.$ $=0.65$ ), and with the noise power spectrum shown in Fig. 4(b). The parameters are described in Sec. IV C. We break the parameter estimation to consider the recovery of three, five, and seven parameters. Under "Degradation" we tabulate the degradation factors $(F)_{i i}^{-1 / 2} /\left(F^{-1}\right)_{i i}^{1 / 2}$ due to parameter degeneracies. We also list the parameter errors expected if one were to assume Gaussian sample variance only for the SZ power spectrum and were to ignore the non-Gaussian covariance. The increase in error on individual parameters, with the introduction of the full covariance matrix, ranges from $40 \%$ to nearly $100 \%$.

\begin{tabular}{ccccccccc}
\hline \hline & $\mathrm{N}$ & $T_{0}$ & $T_{1}$ & $T_{\text {evol }}$ & $T_{\text {min }}$ & $f_{0}$ & $f_{1}$ & $f_{\text {evol }}$ \\
\hline 3 & Perfect & 0.04 & & & 0.002 & 0.0004 & & \\
& Planck & 0.79 & & & 0.75 & 0.03 & & \\
\hline 5 & Perfect & 0.13 & 0.02 & 0.06 & 0.05 & 0.002 & & \\
& Planck & 1.39 & 0.41 & 1.22 & 1.37 & 0.05 & & \\
\hline 7 & Perfect & 0.30 & 0.07 & 0.17 & 0.07 & 0.008 & 0.06 & 0.13 \\
& Degradation & 47 & 184 & 133 & 34 & 82 & 240 & 130 \\
& Gaussian & 0.18 & 0.04 & 0.10 & 0.04 & 0.005 & 0.04 & 0.08 \\
& Increase (\%) & 64 & 70 & 77 & 81 & 73 & 60 & 68 \\
\hline \hline
\end{tabular}

primarily due to their assumption that temperature properties are a priori known.

In addition to the parameter degeneracies, the nonGaussianities associated with the SZ effect also increase the errors on parameters. For example, for the seven parameters under discussion here and again for a perfect and full sky experiment, we list the errors in parameters one can obtain if one were to ignore the non-Gaussian contributions to the covariance. As tabulated, non-Gaussianities increase the error on parameters by factors up to 1.5, suggesting that the ignoring the non-Gaussianities will lead to a significant underestimate of the errors in parameters. This should be considered under the context that the SZ effect is significantly non-Gaussian at all scales of interest and that ability to distinguish parameters occurs only at multipoles of a few thousand where the non-Gaussianities in fact dominate.

\section{SZ KINETIC (OSTRIKER-VISHNIAC) EFFECT}

The bulk flow of electrons that scatter the CMB photons, in the reionized epoch, lead to temperature fluctuations through the well known Doppler effect

$$
T^{\mathrm{dop}}(\hat{\mathbf{n}})=\int_{0}^{r_{0}} \operatorname{drg}(r) \hat{\mathbf{n}} \cdot \mathbf{v}_{g}(r, \hat{\mathbf{n}} r),
$$

where $\mathbf{v}_{g}$ is the baryon velocity. In Fig. 4(a), we show the linear Doppler effect, including contributions resulting due to double scattering effect described in Ref. [42] (see Ref. [2] for details). The power spectrum is such that it peaks around the horizon at the reionization projected on the sky today.
The effect cancels out significantly at scales smaller than the horizon at scattering since photons scatter against the crests and troughs of the perturbation.

The Ostriker-Vishniac effect arises from the second-order modulation of the Doppler effect by density fluctuations [19], and avoids strong cancellation associated with the linear Doppler effect. This nonlinear effect is also known as the kinetic SZ effect from a large-scale structure [20] and is associated with the line of sight motion of halos. Due to the density weighting, the kinetic SZ effect peaks at small scales: sub arcminutes for $\Lambda \mathrm{CDM}$. For a fully ionized universe, contributions are broadly distributed in redshift so that the power spectra are moderately dependent on the optical depth $\tau$. Here we assume an optical depth to ionization up to 0.1, consistent with current upper limits on the reionization redshift from CMB [43] and other observational data (see, e.g., Ref. [44] and references therein).

The kinetic SZ temperature fluctuations, denoted as kSZ, can be written as a product of the line of sight velocity, under linear theory, and density fluctuations:

$$
\begin{aligned}
T^{\mathrm{kSZ}}(\hat{\mathbf{n}})= & \int d r g(r) \hat{\mathbf{n}} \cdot \mathbf{v}_{g}(r, \hat{\mathbf{n}} r) \delta_{g}(r, \hat{\mathbf{n}} r) \\
= & -i \int d r g \dot{G} G \int \frac{d^{3} \mathbf{k}}{(2 \pi)^{3}} \int \frac{d^{3} \mathbf{k}^{\prime}}{(2 \pi)^{3}} \\
& \times \delta_{\delta}^{\operatorname{lin}}\left(\mathbf{k}-\mathbf{k}^{\prime}\right) \delta_{g}\left(\mathbf{k}^{\prime}\right) e^{i \mathbf{k} \cdot \hat{\mathbf{n}} r}\left[\hat{\mathbf{n}} \cdot \frac{\mathbf{k}-\mathbf{k}^{\prime}}{\left|\mathbf{k}-\mathbf{k}^{\prime}\right|^{2}}\right] .
\end{aligned}
$$

Here we have used the linear theory to obtain the large scale velocity field in terms of the linear dark matter density field. The multiplication between the velocity and density fields in real space has been converted to a convolution between the two fields in Fourier space.

We can now expand out the temperature perturbation due to the kinetic SZ effect, $T^{\mathrm{kSZ}}$, into spherical harmonics,

$$
\begin{aligned}
& a_{l m}^{\mathrm{kSZ}}=-i \int d \hat{\mathbf{n}} \int d r(g \dot{G} G) \int \frac{d^{3} \mathbf{k}_{1}}{(2 \pi)^{3}} \int \frac{d^{3} \mathbf{k}_{2}}{(2 \pi)^{3}} \\
& \times \delta_{\delta}^{\operatorname{lin}}\left(\mathbf{k}_{1}\right) \delta_{g}\left(\mathbf{k}_{2}\right) e^{i\left(\mathbf{k}_{1}+\mathbf{k}_{2}\right) \cdot \hat{\mathbf{n}} r}\left[\frac{\hat{\mathbf{n}} \cdot \mathbf{k}_{1}}{k_{1}^{2}}\right] Y_{l}^{m *}(\hat{\mathbf{n}}),
\end{aligned}
$$

where we have symmetrizised by using $\mathbf{k}_{1}$ and $\mathbf{k}_{2}$ to represent $\mathbf{k}-\mathbf{k}^{\prime}$ and $\mathbf{k}^{\prime}$, respectively. Using

$$
\hat{\mathbf{n}} \cdot \mathbf{k}=\sum_{m^{\prime}} \frac{4 \pi}{3} k Y_{1}^{m^{\prime}}(\hat{\mathbf{n}}) Y_{1}^{m^{\prime}} *(\hat{\mathbf{k}})
$$

and the Rayleigh expansion

$$
e^{i \mathbf{k} \cdot \hat{\mathbf{n}} r}=4 \pi \sum_{l m} i^{l} j_{l}(k r) Y_{l}^{m *}(\hat{\mathbf{k}}) Y_{l}^{m}(\hat{\mathbf{n}}),
$$

we can further simplify and rewrite the multipole moments as 


$$
\begin{aligned}
a_{l m}^{\mathrm{kSZ}}= & -i \frac{(4 \pi)^{3}}{3} \int d r \int \frac{d^{3} \mathbf{k}_{1}}{(2 \pi)^{3}} \int \frac{d^{3} \mathbf{k}_{2}}{(2 \pi)^{3}} \sum_{l_{1} m_{1}} \sum_{l_{2} m_{2}} \sum_{m^{\prime}} \\
& \times(i)^{l_{1}+l_{2}}(g \dot{G} G) \frac{j_{l_{1}}\left(k_{1} r\right)}{k_{1}} j_{l_{2}}\left(k_{2} r\right) \delta_{\delta}^{\operatorname{lin}}\left(\mathbf{k}_{1}\right) \delta_{g}\left(\mathbf{k}_{2}\right) \\
& \times Y_{l_{1}}^{m_{1}}\left(\hat{\mathbf{k}}_{1}\right) Y_{1}^{m^{\prime}}\left(\hat{\mathbf{k}}_{1}\right) Y_{l_{2}}^{m_{2}}\left(\hat{\mathbf{k}}_{2}\right) I_{l l_{1} l_{2} 1}^{m^{*} m_{2}^{*} m_{2}^{*} m^{\prime *}}(\hat{\mathbf{n}}) .
\end{aligned}
$$

Here and throughout, we make use of the general integral over spherical harmonics written such that

$$
I_{l_{1} l_{2} \ldots l_{i}}^{m_{1} m_{2} \ldots m_{i}}(\hat{\mathbf{n}})=\int d \hat{\mathbf{n}} Y_{l_{1}}^{m_{1}}(\hat{\mathbf{n}}) Y_{l_{2}}^{m_{2}}(\hat{\mathbf{n}}) \ldots Y_{l_{i}}^{m_{i}}(\hat{\mathbf{n}}) .
$$

We can construct the angular power spectrum by considering $\left\langle a_{l_{1} m_{1}} a_{l_{2} m_{2}}^{*}\right\rangle$. Under the assumption that the temperature field is statistically isotropic, the correlation is independent of $m$, and we can write the angular power spectrum as

$$
\left\langle a_{l_{1} m_{1}}^{*, \mathrm{kSZ}} a_{l_{2} m_{2}}^{\mathrm{kSZ}}\right\rangle=\delta_{l_{1} l_{2}}^{\mathrm{D}} \delta_{m_{1} m_{2}}^{\mathrm{D}} C_{l_{1}}^{\mathrm{kSZ}} .
$$

The correlation can be written using

$$
\begin{aligned}
& \left\langle a_{l_{1} m_{1}}^{*, \mathrm{kSZ}} a_{l_{2} m_{2}}^{\mathrm{kSZ}}\right\rangle=\frac{(4 \pi)^{6}}{9} \int d r_{1} g \dot{G} G \int d r_{2} g \dot{G} G \\
& \times \int \frac{d^{3} \mathbf{k}_{1}}{(2 \pi)^{3}} \frac{d^{3} \mathbf{k}_{2}}{(2 \pi)^{3}} \frac{d^{3} \mathbf{k}_{1}^{\prime}}{(2 \pi)^{3}} \frac{d^{3} \mathbf{k}_{2}^{\prime}}{(2 \pi)^{3}} \sum_{l_{1}^{\prime} m_{1}^{\prime} l_{1}^{\prime \prime} m_{1}^{\prime \prime} m_{1}^{\prime \prime \prime} l_{2}^{\prime} m_{2}^{\prime} l_{2}^{\prime \prime} m_{2}^{\prime \prime} m_{2}^{\prime \prime \prime}}\left\langle\delta_{\delta}^{\operatorname{lin}}\left(\mathbf{k}_{1}^{\prime}\right) \delta_{g}\left(\mathbf{k}_{2}^{\prime}\right) \delta_{\delta}^{* \operatorname{lin}}\left(\mathbf{k}_{1}\right) \delta_{g}^{*}\left(\mathbf{k}_{2}\right)\right\rangle \\
& \times(-i)^{l_{1}^{\prime}+l_{1}^{\prime \prime}}(i)_{2}^{l_{2}^{\prime}+l_{2}^{\prime \prime}} j_{l_{2}^{\prime}}\left(k_{1}^{\prime} r_{2}\right) \frac{j_{l_{2}^{\prime \prime}}\left(k_{2}^{\prime} r_{2}\right)}{k_{2}^{\prime}} \frac{j_{l_{1}^{\prime}}\left(k_{1} r_{1}\right)}{k_{1}} j_{l_{1}^{\prime \prime}}\left(k_{2} r_{1}\right) \\
& \times Y_{l_{2}^{\prime}}^{m_{2}^{\prime}}\left(\hat{\mathbf{k}}_{1}^{\prime}\right) Y_{1}^{m_{2}^{\prime \prime \prime}}\left(\hat{\mathbf{k}}_{2}^{\prime}\right) Y_{l_{2}^{\prime \prime}}^{m_{2}^{\prime \prime}}\left(\hat{\mathbf{k}}_{1}^{\prime}\right) Y_{l_{1}^{\prime}}^{m_{1}^{\prime} *}\left(\hat{\mathbf{k}}_{1}\right) Y_{1}^{m_{1}^{\prime \prime \prime *} *}\left(\hat{\mathbf{k}}_{1}\right) Y_{l_{1}^{\prime \prime}}^{m_{1}^{\prime \prime *}}\left(\hat{\mathbf{k}}_{2}\right) I_{l_{2} l_{2}^{\prime} l_{2}^{\prime \prime} 1}^{m_{2} m_{2}^{\prime} * m_{2}^{\prime \prime *} m_{2}^{\prime \prime \prime *} *}(\hat{\mathbf{m}}) I_{l_{1} l_{1}^{\prime} l_{1}^{\prime \prime} 1}^{m_{1}^{*} m_{1}^{\prime} m_{1}^{\prime \prime} m_{2}^{\prime \prime \prime}}(\hat{\mathbf{n}}) \text {. }
\end{aligned}
$$

We can separate out the contributions such that the total is made of correlations following $\left\langle v_{g} v_{g}\right\rangle\left\langle\delta_{g} \delta_{g}\right\rangle$ and $\left\langle v_{g} \delta_{g}\right\rangle\left\langle v_{g} \delta_{g}\right\rangle$ depending on whether we consider cumulants by combining $\mathbf{k}_{1}$ with $\mathbf{k}_{1}^{\prime}$ or $\mathbf{k}_{2}^{\prime}$ respectively. After some straightforward but tedious algebra, and noting that

$$
\sum_{m_{1}^{\prime} m_{2}^{\prime}}\left(\begin{array}{ccc}
l_{1}^{\prime} & l_{2}^{\prime} & l_{1} \\
m_{1}^{\prime} & m_{2}^{\prime} & m_{1}
\end{array}\right)\left(\begin{array}{ccc}
l_{1}^{\prime} & l_{2}^{\prime} & l_{2} \\
m_{1}^{\prime} & m_{2}^{\prime} & m_{2}
\end{array}\right)=\frac{\delta_{m_{1} m_{2}} \delta_{l_{1} l_{2}}}{2 l_{1}+1},
$$

we can write

$$
\begin{aligned}
C_{l}^{\mathrm{kSZ}=} & \frac{2^{2}}{\pi^{2}} \sum_{l_{1} l_{2}}\left[\frac{\left(2 l_{1}+1\right)\left(2 l_{2}+1\right)}{4 \pi}\right]\left(\begin{array}{ccc}
l & l_{1} & l_{2} \\
0 & 0 & 0
\end{array}\right)^{2} \int d r_{1} g \dot{G} G \int d r_{2} g \dot{G} G \int k_{1}^{2} d k_{1} \int k_{2}^{2} d k_{2} \\
& \times\left(P_{\delta \delta}^{\operatorname{lin}}\left(k_{1}\right) P_{g g}\left(k_{2}\right) j_{l_{1}}\left(k_{2} r_{2}\right) j_{l_{1}}\left(k_{2} r_{1}\right) \frac{j_{l_{2}}^{\prime}\left(k_{1} r_{1}\right)}{k_{1}} \frac{j_{l_{2}}^{\prime}\left(k_{1} r_{2}\right)}{k_{1}}+P_{\delta g}\left(k_{1}\right) P_{\delta g}\left(k_{2}\right) j_{l_{2}}\left(k_{2} r_{1}\right) \frac{j_{l_{1}}^{\prime}\left(k_{1} r_{1}\right)}{k_{1}} j_{l_{1}}\left(k_{1} r_{2}\right) \frac{j_{l_{2}}^{\prime}\left(k_{2} r_{2}\right)}{k_{2}}\right) .
\end{aligned}
$$

Here the first term represents the contribution from $\left\langle v_{g} v_{g}\right\rangle\left\langle\delta_{g} \delta_{g}\right\rangle$, while the second term is the $\left\langle v_{g} \delta_{g}\right\rangle\left\langle v_{g} \delta_{g}\right\rangle$ contribution, respectively. In simplifying the integrals involving spherical harmonics, we have made use of the properties of Clebsch-Gordan coefficients, in particular, those involving $l=1$. The integral involves two distances and two Fourier modes and is summed over the Wigner $3 j$ symbol to obtain the power spectrum. The above equation represents the angular power spectrum of the kinetic SZ or OV effect, under the all-sky coordinates; we have not used the flat-sky assumption or any small angular scale limit which is usually considered in the literature.
Since we are primarily interested in the contribution at small angular scales here, we can ignore the contribution to the kinetic SZ effect involving the correlation between linear density field and baryons, and consider only the contribution that results from baryon-baryon and density-density correlations. In fact, under the halo description provided here, there is no correlation of the baryon field within halos and the velocity field traced by individual halos. Thus, contribution to the baryon-velocity correlation only comes from the twohalo term of the density field-baryon correlation. This correlation is suppressed at small scales and is not a significant contributor to the kinetic SZ power spectrum (see Ref. [20]). 
Similarly to the Limber approximation [37], in order to simplify the calculation associated with $\left\langle v_{g} v_{g}\right\rangle\left\langle\delta_{g} \delta_{g}\right\rangle$, we use an equation involving completeness of spherical Bessel functions,

$$
\left.\int d k k^{2} F(k) j_{l}(k r) j_{l}\left(k r^{\prime}\right) \approx \frac{\pi}{2} d_{A}^{-2} \delta^{\mathrm{D}}\left(r-r^{\prime}\right) F(k)\right|_{k=l l d_{A}},
$$

where the assumption is that $F(k)$ is a slowly varying function. Applying this to the integral over $k_{2}$ gives

$$
\begin{aligned}
C_{l}^{\mathrm{kSZ}}= & \frac{2}{\pi} \sum_{l_{1} l_{2}}\left[\frac{\left(2 l_{1}+1\right)\left(2 l_{2}+1\right)}{4 \pi}\right]\left(\begin{array}{ccc}
l & l_{1} & l_{2} \\
0 & 0 & 0
\end{array}\right)^{2} \\
& \times \int d r_{1} \frac{(g \dot{G})^{2}}{d_{A}^{2}} \int k_{1}^{2} d k_{1} P_{\delta \delta}^{\operatorname{lin}}\left(k_{1}\right) P_{g g}\left[\frac{l_{1}}{d_{A}} ; r_{1}\right] \\
& \times\left(\frac{j_{l_{2}}^{\prime}\left(k_{1} r_{1}\right)}{k_{1}}\right)^{2} .
\end{aligned}
$$

An alternative approach, which has been the calculational method in many of the previous papers $[19,45,20]$, is to use a specific coordinate frame with the $z$ axis along $\overrightarrow{\mathbf{k}}$. This allows one to simplify the SZ kinetic power spectrum to

$$
C_{l}^{\mathrm{kSZ}}=\frac{1}{8 \pi^{2}} \int d r \frac{(g \dot{G} G)^{2}}{d_{A}^{2}} P_{\delta \delta}(k)^{2} I_{v}\left(k=\frac{l}{d_{A}}\right),
$$

with the mode-coupling integral given by

$$
\begin{aligned}
I_{v}(k)= & \int d k_{1} \int_{-1}^{+1} d \mu \frac{\left(1-\mu^{2}\right)\left(1-2 \mu y_{1}\right)}{y_{2}^{2}} \frac{P_{\delta \delta}\left(k y_{1}\right)}{P_{\delta \delta}(k)} \\
& \times \frac{P_{\delta \delta}\left(k y_{2}\right)}{P_{\delta \delta}(k)} .
\end{aligned}
$$

We refer the reader to Ref. [45] for details on this derivation. In the above, $\mu=\hat{\mathbf{k}} \cdot \hat{\mathbf{k}}_{1}, \quad y_{1}=k_{1} / k$ and $y_{2}=k_{2} / k$ $=\sqrt{1-2 \mu y_{1}+y_{1}^{2}}$. This flat-sky approximation makes use of the Limber approximation [37] to further simplify the calculation with the replacement of $k=l / d_{A}$. The power spectra here represent the baryon field power spectrum and the velocity field power spectrum; the former assumed to trace the dark matter density field while the latter is generally related to the linear dark matter density field through the use of linear theory arguments.

The correspondence between the flat-sky and all-sky formulations can be obtained by noting that in the small scale limit contributions to the flat-sky effect comes when $k_{2}=\mid \mathbf{k}$ $-\mathbf{k}_{1} \mid \sim k$, such that $y_{1} \ll 1$. In this limit, the flat sky OstrikerVishniac effect reduces to a simple form given by Hu in Ref. [20]:

$$
C_{l}^{\mathrm{kSZ}}=\frac{1}{3} \int d r \frac{(g \dot{G} G)^{2}}{d_{A}^{2}} P_{g g}(k) v_{\mathrm{rms}}^{2} .
$$

Here $v_{\text {rms }}^{2}$ is the rms of the uniform bulk velocity from large scales:

$$
v_{\mathrm{rms}}^{2}=\int d k \frac{P_{\delta \delta}(k)}{2 \pi^{2}}
$$

The $1 / 3$ arises from the fact that $\mathrm{rms}$ in each component is one-third of the total velocity.

In the same small scale limit, to be consistent with the flat sky expression, we can reduce the all-sky expression such that contributions come from a term that looks like

$$
C_{l}^{\mathrm{kSZ}}=\int d r \frac{(g \dot{G})^{2}}{d_{A}^{2}} P_{g g}\left[\frac{l}{d_{A}} ; r_{1}\right] \frac{1}{3} v_{\mathrm{rms}}^{2}
$$

A comparison of the reduced all-sky [Eq. (79)] and flatsky (Eq. 82) formulas in the small-scale limit suggests that the correspondence between the two arises when

$$
\sum_{l_{1} l_{2}}\left(2 l_{1}+1\right)\left(2 l_{2}+1\right)\left(\begin{array}{ccc}
l & l_{1} & l_{2} \\
0 & 0 & 0
\end{array}\right)^{2}\left[j_{l_{2}}^{\prime}(k r)\right]^{2}=\frac{1}{3}
$$

Numerically, we determined this to be true as long as $l_{2} \ll l$; however, we have not been able to prove this relation analytically. We leave this as a challenge to our readers. Note that the limit $l_{2} \ll l$ is the small-scale limit in the flat-sky and is effectively equivalent to $y_{1} \ll 1$ considered in writing Eq. (82). Thus the right hand side of the above expression denotes the all-sky equivalent of the integral that leads to onethird of a rms of a randomly directed quantity along one particular line of sight.

\section{Discussion}

In Fig. 4, we show our prediction for the SZ kinetic effect and a comparison with the SZ thermal contribution. As shown, the SZ kinetic contribution is roughly an order of magnitude smaller than the kinetic SZ contribution. There is also a more fundamental difference between the two: the SZ thermal effect, due to its dependence on the highest temperature electrons, is more dependent on the most massive halos in the universe, while the SZ kinetic effect arises more clearly due to large scale correlations of the halos that form a large scale structure. The difference arises from that fact that kinetic SZ effect is mainly due to baryons and not the temperature weighted baryons that trace the pressure responsible for the thermal effect. Contributions to the SZ kinetic effect come from baryons tracing all scales and down to small mass halos. The difference associated with mass dependence between the two effects suggests that a wide-field SZ thermal effect map and a wide-field SZ kinetic effect map will be different from each other in that massive halos, or clusters, will be clearly visible in a SZ thermal map while the large scale structure will be more evident in a SZ kinetic effect map. Numerical simulations are in fact consistent with this picture [14].

As shown in Fig. 4(b), the variations in maximum mass used in the calculation do not lead to orders of magnitude 


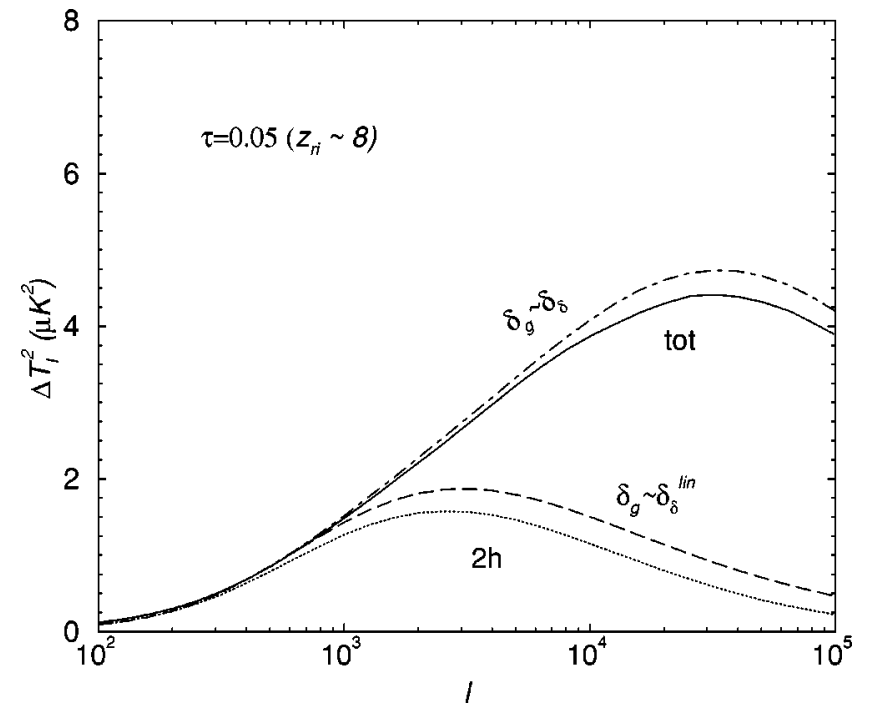

FIG. 10. The temperature fluctuation power $\left[\Delta T_{l}^{2}=l(l\right.$ $\left.+1) /(2 \pi) C_{l} T_{\mathrm{CMB}}^{2}\right]$ for a variety of methods to calculate the kinetic $\mathrm{SZ}$ effect. Here we show the contribution for a reionization redshift of $\sim 8$ and an optical depth to reionization of 0.05 . The contributions are calculated under the assumption that the baryon field traces the nonlinear dark matter $\left[P_{g}(k)=P_{\delta}(k)\right.$ with $P_{\delta}(k)$ predicted by the halo model], the linear density field $\left[P_{g}(k)\right.$ $\left.=P^{\operatorname{lin}}(k)\right]$, and the halo model for gas, with total and the two-halo contributions shown separately. For the most part, the kinetic SZ effect can be described using linear theory, and the nonlinearities only increase the temperature fluctuation power by a factor of a few at $l \sim 10^{5}$.

changes in the total kinetic SZ contribution. This change is less than the changes in the total thermal SZ contribution as a function of maximum mass. This is again consistent with our basic result that most contributions come from the large scale linear velocity modulated by baryons in halos. Consequently, while the thermal SZ effect is dominated by shotnoise contributions, and is heavily affected by the sample variance, the same is not true for the kinetic SZ effect.

In Fig. 10, we show several additional predictions for the kinetic SZ effect, following the discussion in Ref. [20]. Here we calculate the kinetic SZ power spectrum under several assumptions, including the case when gas is assumed to trace the nonlinear density field and the linear density field. We compare predictions based on such assumptions to those calculated using the halo model. As shown, the halo model calculation shows slightly less power than when using the nonlinear dark matter density field to describe clustering of baryons. This difference arises from the fact that baryons do not fully trace the dark matter in halos. Due to small differences, one can safely use the nonlinear dark matter power spectrum to describe baryons. Using the linear theory only, however, leads to an underestimate of power at a factor of $3-4$ at scales corresponding to multipoles of $l \sim 10^{4}$ to $10^{5}$, and may not provide an accurate description of the total kinetic SZ effect.

The interesting experimental possibility here is whether one can obtain a wide-field map of the SZ kinetic effect. Since it is now well known that the unique spectral depen- dence of the thermal SZ effect can be used to separate its contribution [10] at smaller angular scales, it is likely that, after the separation, the SZ kinetic effect will be the dominant signal, even after accounting for the lensed CMB contribution. For such a separation of the SZ thermal effect to be carried out and such that a detection of the kinetic SZ effect will be possible, observations, at multifrequencies, are needed to arcminute scales. Upcoming interferometers and similar experiments will allow such studies to be eventually carried out. A wide-field kinetic SZ map of the large scale structure will eventually allow an understating of the large scale velocity field of baryons, as the density fluctuations can be identified through a cross-correlation of such a map with a similar thermal SZ map. We now discuss the existence of correlations between the SZ thermal and SZ kinetic effect.

\section{SZ THERMAL-SZ KINETIC CORRELATION}

The SZ thermal and SZ kinetic effects both trace the large scale structure baryons. One can study a correlation between these two effects to probe the manner in which baryons are distributed in the large scale structure. For example, such a correlation study may allow one to answer to what extent diffuse baryons contribute to thermal SZ effect when compared to their contribution to kinetic SZ effect. Given that the SZ kinetic effect is second order in fluctuations, there is no direct two-point correlation function between the temperature anisotropies produced by SZ thermal and kinetic effects. As discussed in Ref. [2], to lowest order, the correlation between kinetic SZ and thermal SZ effects manifests itself as a nonvanishing bispectrum in temperature fluctuations and can be studied by considering a three-point correlation function or a bispectrum, the Fourier space analog of the three point function, or associated statistics such as the third moment or skewness.

\section{A. SZ thermal-SZ thermal-SZ kinetic bispectrum}

We refer the reader to Ref. [2] for full details on the derivation of the SZ thermal-SZ kinetic correlation as a bispectrum involving two measurements of $S Z$ thermal effect and one measurement of SZ kinetic effect (SZ thermal-SZ thermal-SZ kinetic effect); note that in Ref. [2], we identified this bispectrum as the SZ-SZ-OV bispectrum. As discussed there, for low optical depths to reionization, the detection of the SZ-SZ-OV bispectrum is problematic due to high cosmic variance resulting from primary anisotropies.

In Ref. [2], we assumed that pressure and baryons both trace dark matter to calculate the SZ-SZ-OV bispectrum. Using our halo model, we can now update this calculation to include the bispectrum formed between SZ thermal-SZ thermal-SZ kinetic effects. Additionally, we can investigate the improvements in the signal-to-noise ratio for the bispectrum detections when the SZ thermal effect is separated from the CMB effect. The maximum signal-to-noise ratio for this bispectrum can only be achieved when the SZ kinetic effect is also separated from the CMB effect though, given that the two effects have the same frequency dependence, it is unlikely that the kinetic SZ effect can be separated from thermal CMB anisotropies using frequency information alone. 
However, using the frequency information, with the thermal SZ contribution separated from the CMB contribution, it is likely that the SZ kinetic effect will dominate the small angular scale signal in the temperature anisotropies. Thus one can use small angular scale thermal CMB temperature fluctuations to construct the SZ thermal-SZ kinetic bispectrum.

Following Ref. [2], we can write the SZ thermal-SZ thermal-SZ kinetic bispectrum as

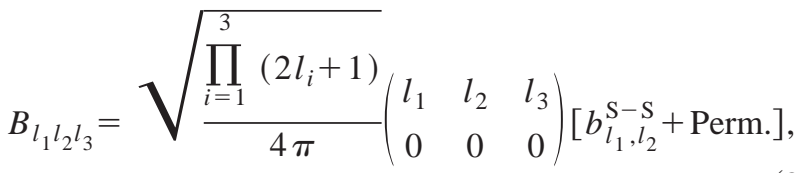

with

$$
\begin{aligned}
b_{l_{1}, l_{2}}^{\mathrm{SZ}-\mathrm{SZ}=}= & \frac{2}{\pi} \int \frac{d r}{d_{A}^{2}} W^{\mathrm{SZ}}(r) g P_{g \Pi}\left(\frac{l_{2}}{d_{A}} ; r\right) \\
& \times \int d r_{1} \int k_{1} d k_{1} P_{\delta \Pi}^{2 h}\left(k_{1} ; r_{1}\right) W^{\mathrm{SZ}} \\
& \times\left(r_{1}\right) j_{l_{1}}^{\prime}\left(k_{1} r_{1}\right) j_{l_{1}}\left(k_{1} r\right) .
\end{aligned}
$$

Here "Perm." means a sum over the remaining five permutations of $\left(l_{1}, l_{2}, l_{3}\right)$ as usual.

Here $P_{g \Pi}$ is the baryon-pressure power spectrum while the $P_{\delta \Pi}$ is the density-pressure power spectrum, with $\delta$ tracing the linear velocity field. Since there is no contribution to the large scale bulk flows from the non-linear regime (i.e. the one-halo term), we model the pressure-density cross power as the large scale density-pressure correlations in the linear regime described by the two-halo term.

The signal-to-noise ratio for the detection of the bispectrum is

$$
\left(\frac{S}{N}\right)^{2} \equiv \frac{1}{\sigma^{2}(A)}=\sum_{l_{3} \geqslant l_{2} \geqslant l_{1}} \frac{B_{l_{1} l_{2} l_{3}}^{2}}{C_{l_{1}}^{\mathrm{t}} C_{l_{2}}^{\mathrm{t}} C_{l_{3}}^{\mathrm{t}}}
$$

where

$$
C_{l}^{\mathrm{t}}=C_{l}^{\mathrm{CMB}}+C_{l}^{\mathrm{sec}}+C_{l}^{\mathrm{Noise}} .
$$

In Ref. [10], we showed how multifrequency cleaning of the SZ effect can be a useful tool for higher order correlation studies, and discussed how the signal-to-noise ratio for the detection of SZ-lensing correlation, again through a bispectrum, can be improved by using a CMB primary anisotropy separated SZ map. We consider the same approach here, where we study the possibility for a detection of the SZ thermal-SZ kinetic correlation by using a frequency cleaned SZ thermal map, which will provide two measurements, and a CMB map containing only the CMB primary contribution, SZ kinetic contribution, and thermal-like secondary contribution.

In Fig. 11(a), we update signal-to-noise results for the bispectrum given in Ref. [2], where we only studied the possible detection in CMB data alone and with no consideration

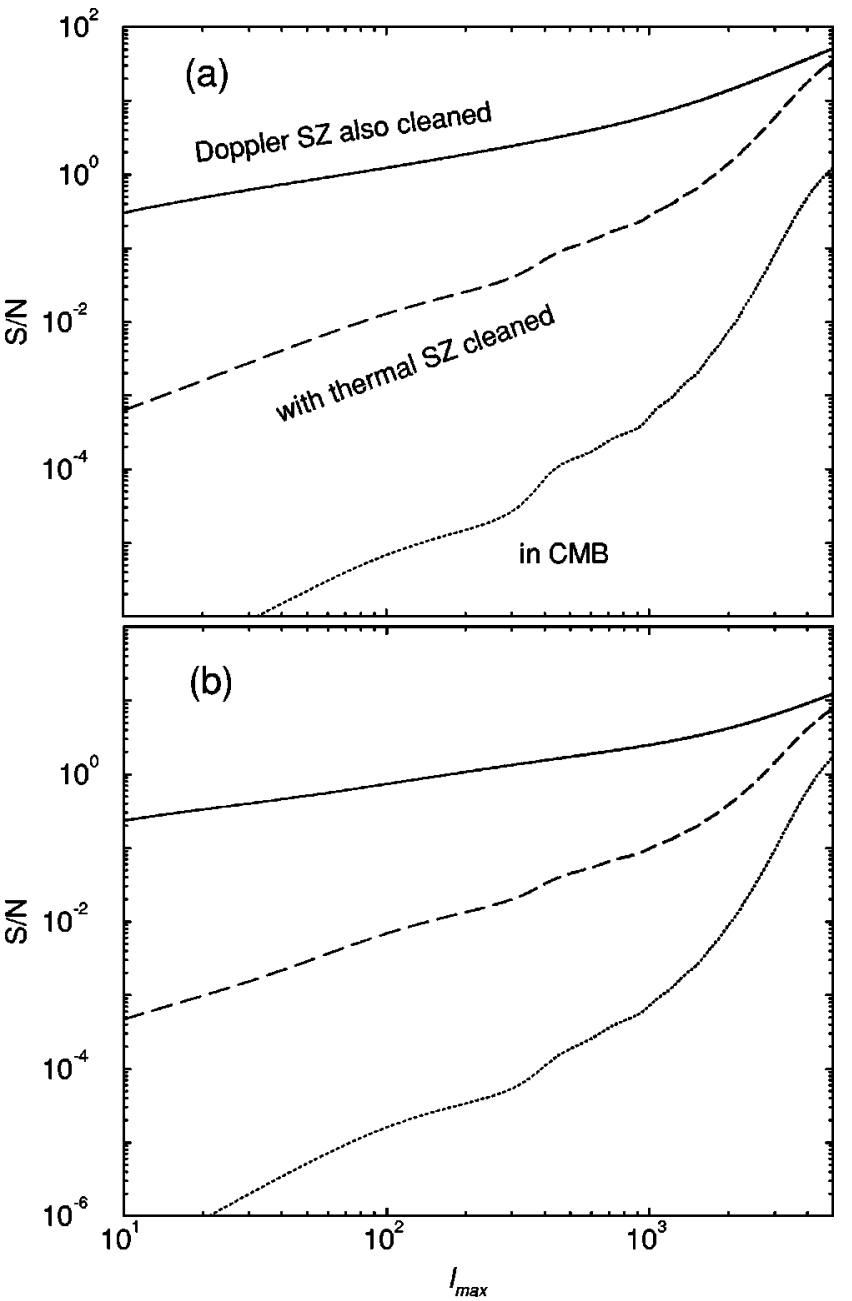

FIG. 11. Cumulative signal-to-noise ratio for the detection of the SZ thermal-SZ thermal-SZ kinetic bispectrum (a) and skewness (b) with temperature anisotropy data. The dotted line is for the detection of SZ thermal-SZ kinetic correlation using CMB data alone, while the dashed line is the same when the SZ thermal effect has been separated from other CMB contributions and the measurement now involves two points from the SZ map and one point from the CMB. Finally, the solid line is the maximum signal-to-noise ratio achievable with the separation of the SZ kinetic effect from all contributors to CMB anisotropy.

of separation of effects, especially the SZ thermal effect. The separation allows a decrease in cosmic variance, as the noise is no longer dominated by CMB primary anisotropies. This leads to an increase in the cumulative signal-to-noise. With the SZ thermal effect separated, we see that the signal-tonoise ratio increases by roughly two orders of magnitude. In Ref. [10], we showed how one can obtain an order of magnitude in signal-to-noise ratio when a CMB separated SZ thermal map is used for a detection of SZ-lensing correlation. Here we obtain an improvement of roughly two orders of magnitude, since the SZ thermal-SZ kinetic correlation is present with two SZ thermal measurements, instead of one in the case SZ thermal-lensing correlation through the SZ thermal-CMB-CMB bispectrum.

If one can separate the SZ kinetic such that a perfect SZ 
kinetic map as well as a perfect SZ thermal map are available, then one can improve the signal-to-noise ratio for detection significantly such that a detection is possible. Since the SZ kinetic effect is expected to dominate temperature anisotropies at small angular scales, when the SZ thermal effect is removed, an opportunity to detect the SZ thermal-SZ kinetic correlation will likely come from small angular scale multifrequency experiments. One can also improve the possibility of detecting this correlation by noting that the configuration for the bispectrum is such that it peaks for highly flattened triangles (see Ref. [2]). It is likely that progress in experimental studies will continue to a level where such studies will eventually be possible.

Since the bispectrum may be hard to measure directly from observational data, we also consider a real space statistic that probes the non-Gaussian information at the three point level. The simplest aspect of the bispectrum that can be measured in real space is the third moment of the map smoothed on some scale with a window $W(\sigma)$,

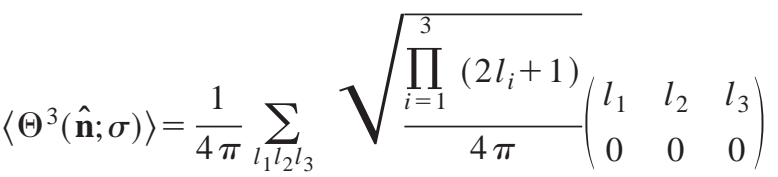

$$
\begin{aligned}
& \times B_{l_{1} l_{2} l_{3}} W_{l_{1}}(\sigma) W_{l_{2}}(\sigma) W_{l_{3}}(\sigma),
\end{aligned}
$$

where $W_{l}$ are the multipole moments, or Fourier transforms in a flat-sky approximation, of the window. Note that the skewness is $s_{3}=\left\langle\Theta^{3}(\hat{\mathbf{n}} ; \sigma)\right\rangle /\left\langle\Theta^{2}(\hat{\mathbf{n}} ; \sigma)\right\rangle^{2}$.

The overall signal-to-noise ratio for the measurement of the third moment is

$$
\left(\frac{S}{N}\right)^{2}=f_{\text {sky }} \frac{\left\langle\Theta^{3}(\hat{\mathbf{n}} ; \sigma)\right\rangle^{2}}{\operatorname{Var}}
$$

where the variance, assuming Gaussian statistics, is given by

$$
\begin{aligned}
\operatorname{Var}= & \frac{1}{(4 \pi)^{2}} \sum_{l_{1} l_{2} l_{3}} \frac{\prod_{i=1}^{3}\left(2 l_{i}+1\right)}{4 \pi}\left(\begin{array}{ccc}
l_{1} & l_{2} & l_{3} \\
0 & 0 & 0
\end{array}\right)^{2} \\
& \times W_{l_{1}}^{2}(\sigma) W_{l_{2}}^{2}(\sigma) W_{l_{3}}^{2}(\sigma) 6 C_{l_{1}}^{\mathrm{t}} C_{l_{2}}^{\mathrm{t}} C_{l_{3}}^{\mathrm{t}} .
\end{aligned}
$$

In Fig. 11(b), we show the signal-to-noise ratio for the detection of the third moment. Here we use a top-hat window in multipole space out to $l_{\max }$, so that a direct comparison is possible with the signal-to-noise calculation involving the bispectrum. As shown, we find that there is a lower signalto-noise ratio in the skewness when compared to the full bispectrum. This results from the fact that the bispectrum contains all information at the three point level, while a measurement of the third moment leads to a loss of information. This can also be understood by noting that the signal-tonoise ratio for the bispectrum and skewness is such that in the case of the bispectrum the signal-to-noise ratio is calculated for each mode and summed up, while for the skewness the signal-to-noise ratio is calculated after summing the signal and noise separately over all modes.

\section{B. SZ thermal ${ }^{2}-\mathrm{SZ}$ kinetic $^{2}$ power spectrum}

In addition to the SZ thermal-SZ kinetic-SZ kinetic bispectrum, we can introduce higher order correlations involving SZ thermal and SZ kinetic effects that probe the correlation between the two. One such possibility is the trispectrum formed by the SZ thermal and SZ kinetic effects. The experiemental measurement of the full trispectrum, however, is likely to be challenging given the number of modes involved and the low signal-to-noise ratio of each of the modes; the same problem is also present at the three point level when measuring the full bispectrum, but estimators such as the third moment or the skewness can capture partial information from the three-point correlations.

Here we focus on a statistic that captures the correlation information coming from higher order, essentially from a trispectrum, but is easily measurable in experimental data since it only involves a power spectrum. Such a possibility involves the power spectrum of squared temperatures instead of the usual temperature itself. Our motivation for such a statistic came when we inspected the published maps of the large scale SZ thermal and SZ kinetic effects in simulations in Ref. [14], and realized that there is a significant correlation between the two effects. Since the temperature fluctuations produced by the SZ kinetic effect oscillate between positive and negative values depending on the direction of the velocity field along the line of sight, as stated earlier, a direct two point correlation involving the temperature results in no contribution. A nonzero correlation between SZ thermal and SZ kinetic effects still manifests if the absolute value of the temperature fluctuation due to the kinetic SZ effect is considered. Since the absolute value of the temperature is equivalent to squaring the temperature, we consider the crosscorrelation of SZ thermal and SZ kinetic effects involving the power spectrum of squared temperatures here.

In order to calculate the SZ thermal ${ }^{2}-\mathrm{SZ}$ kinetic $^{2}$ power spectrum, we can take the all-sky approach presented to describe the SZ kinetic effect. We first note that the spherical harmonic coefficient of the squared can be written through a convolution of the spherical moments of the fluctuations,

$$
\begin{aligned}
a_{l m}^{2} & =\int d \hat{\mathbf{n}} Y_{l}^{* m} T^{2}(\hat{\mathbf{n}}) \\
& =\sum_{l_{1} m_{1}} \sum_{l_{2} m_{2}} a_{l_{1} m_{1}} a_{l_{2} m_{2}}^{*} I_{l l_{1} l_{2}}^{m * m_{1} m_{2}^{*}}(\hat{\mathbf{n}}),
\end{aligned}
$$

where

$$
T(\hat{\mathbf{n}})=\sum_{l m} a_{l m} Y_{l}^{* m} .
$$

Note that the integral over three spherical harmonic coefficients can be written through the use of the Gaunt integral.

Using this identity, we can now construct the power spectrum of thermal $\mathrm{SZ}^{2}$-Doppler $\mathrm{SZ}^{2}$ as 


$$
\begin{aligned}
\left\langle a_{l m}^{\mathrm{kSZ}} a_{l^{\prime} m^{\prime}}^{* S S Z^{2}}\right\rangle= & C_{l}^{2 \mathrm{kSZ}-\mathrm{SZ}} \delta_{l, l^{\prime}} \delta_{m, m^{\prime}} \\
= & \sum_{l_{1} m_{1} l_{2} m_{2}} \sum_{l_{3} m_{3} l_{4} m_{4}}\left\langle a_{l_{1} m_{1}}^{\mathrm{kSZ}} a_{l_{2} m_{2}}^{* \mathrm{kSZ}} a_{l_{3} m_{3}}^{* S Z} a_{l_{4} m_{4}}^{S Z}\right\rangle^{\mathrm{t}} \\
& \times I_{l l_{1} l_{2}}^{m^{*} m_{1} m_{2}^{*}}(\hat{\mathbf{n}}) I_{l^{\prime} l_{3} l_{4}}^{m^{\prime} m_{3}^{*} m_{4}}(\hat{\mathbf{m}}),
\end{aligned}
$$

involving two cumulants of the SZ thermal and SZ Doppler effects, respectively. as

After introducing the multipole moments of the SZ effect

$$
\begin{aligned}
a_{l m}^{\mathrm{SZ}} & =i^{l} \int \frac{d^{3} \mathbf{k}}{2 \pi^{2}} \Pi(\mathbf{k}) Y_{l}^{m} *(\hat{\mathbf{k}}) I_{l}^{\mathrm{SZ}}(k), \\
I_{l}^{\mathrm{SZ}}(k) & =\int d r W^{\mathrm{sZ}}(r) j_{l}(k r),
\end{aligned}
$$

and using the multipole moments of the Doppler SZ effect from Eq. (72), we can write the cumulant involving the four moments as

$$
\begin{aligned}
& \left\langle a_{l_{1} m_{1}}^{\mathrm{kSZ}} a_{l_{2} m_{2}}^{* \mathrm{kSZ}} a_{l_{3} m_{3}}^{* S Z} a_{l_{4} m_{4}}^{S Z}\right\rangle=\frac{(4 \pi)^{8}}{9} \int d r_{1} \cdots \int d r_{4} \int \frac{d^{3} \mathbf{k}_{\mathbf{1}}}{(2 \pi)^{3}} \cdots \int \frac{d^{3} \mathbf{k}_{6}}{(2 \pi)^{3}} \sum_{l_{1}^{\prime} m_{1}^{\prime} l_{1}^{\prime \prime} m_{1}^{\prime \prime} m_{1}^{\prime \prime \prime}} \sum_{l_{2}^{\prime} m_{2}^{\prime} l_{2}^{\prime \prime} m_{2}^{\prime \prime} m_{2}^{\prime \prime \prime}} i l_{1}^{\prime}+l_{1}^{\prime \prime}+l_{4}(-i)^{l_{2}^{\prime}+l_{2}^{\prime \prime}+l_{3}} \\
& \times(g \dot{G} G)_{r_{1}}(g \dot{G} G)_{r_{2}} W^{\mathrm{sz}}\left(r_{3}\right) W^{\mathrm{sz}}\left(r_{4}\right)\left\langle\delta_{\delta}\left(\mathbf{k}_{1}\right) \delta_{b}\left(\mathbf{k}_{2}\right) \delta_{\delta}\left(\mathbf{k}_{3}\right) \delta_{b}\left(\mathbf{k}_{4}\right) \delta_{\Pi}\left(\mathbf{k}_{5}\right) \delta_{\Pi}\left(\mathbf{k}_{6}\right)\right\rangle \\
& \times \frac{j_{l_{1}^{\prime}}\left(k_{1} r_{1}\right)}{k_{1}} j_{l_{1}^{\prime \prime}}\left(k_{2} r_{1}\right) \frac{j_{l_{2}^{\prime}}\left(k_{3} r_{2}\right)}{k_{3}} j_{l_{2}^{\prime \prime}}\left(k_{4} r_{2}\right) j_{l_{3}}\left(k_{5} r_{3}\right) j_{l_{4}}\left(k_{6} r_{4}\right) Y_{l_{1}^{\prime}}^{m_{1}^{\prime}}\left(\hat{\mathbf{k}}_{1}\right) Y_{1}^{m_{1}^{\prime \prime \prime}}\left(\hat{\mathbf{k}}_{1}\right) Y_{l_{1}^{\prime \prime}}^{m_{1}^{\prime \prime}}\left(\hat{\mathbf{k}}_{2}\right) \\
& \times Y_{l_{2}^{\prime}}^{m_{2}^{\prime}}\left(\hat{\mathbf{k}}_{3}\right) Y_{1}^{m_{2}^{\prime \prime \prime}}\left(\hat{\mathbf{k}}_{3}\right) Y_{l_{2}^{\prime \prime}}^{m_{2}^{\prime \prime}}\left(\hat{\mathbf{k}}_{4}\right) Y_{l_{3}}^{m_{3}}\left(\hat{\mathbf{k}}_{5}\right) Y_{l_{4}}^{m_{4}}\left(\hat{\mathbf{k}}_{6}\right) I_{l_{1} l_{1}^{\prime} l_{1}^{\prime \prime} m^{*} m_{1}^{\prime} * m_{1}^{\prime \prime *} m_{1}^{\prime \prime \prime} *}(\hat{\mathbf{n}}) I_{l_{2} l_{2}^{\prime} l_{2}^{\prime \prime} 1}^{m^{*} m_{2}^{\prime} * m_{2}^{\prime \prime} * m_{2}^{\prime \prime \prime} *}(\hat{\mathbf{m}})
\end{aligned}
$$

The cumulant involving six density, baryon and pressure fluctuations can be broken up into two parts involving a Gaussian term, with contributions coming from power spectra of velocities and pressure-density correlations, and a non-Gaussian term, with the velocity power spectrum and the pressure-pressure-baryon-baryon trispectrum. Here we ignore the correlations between pressure and velocity or between baryons and velocities as the scales for such correlations do not match, especially in the small angular scale of interest here. Thus we write

$$
\begin{aligned}
\left\langle\delta_{\delta}\left(\mathbf{k}_{1}\right) \delta_{b}\left(\mathbf{k}_{2}\right) \delta_{\delta}\left(\mathbf{k}_{3}\right) \delta_{b}\left(\mathbf{k}_{4}\right) \delta_{\Pi}\left(\mathbf{k}_{5}\right) \delta_{\Pi}\left(\mathbf{k}_{6}\right)\right\rangle= & \left\langle\delta_{\delta}\left(\mathbf{k}_{1}\right) \delta_{\delta}\left(\mathbf{k}_{3}\right)\right\rangle\left\langle\delta_{b}\left(\mathbf{k}_{2}\right) \delta_{\Pi}\left(\mathbf{k}_{5}\right)\right\rangle\left\langle\delta_{b}\left(\mathbf{k}_{4}\right) \delta_{\Pi}\left(\mathbf{k}_{6}\right)\right\rangle \\
& +\left\langle\delta_{\delta}\left(\mathbf{k}_{1}\right) \delta_{\delta}\left(\mathbf{k}_{3}\right)\right\rangle\left\langle\delta_{b}\left(\mathbf{k}_{2}\right) \delta_{\Pi}\left(\mathbf{k}_{5}\right) \delta_{b}\left(\mathbf{k}_{4}\right) \delta_{\Pi}\left(\mathbf{k}_{6}\right)\right\rangle .
\end{aligned}
$$

We first discuss the Gaussian piece given in the first line above. This term, and a permutation, contributes to the crosscorrelation, and involves the linear density field power spectrum and the nonlinear cross-correlation between baryon and pressure fields. Keeping track of the permutation, and after several simplifications, we write

$$
\begin{aligned}
& \left\langle a_{l_{1} m_{1}}^{\mathrm{kSZ}} a_{l_{2} m_{2}}^{* \mathrm{kSZ}} a_{l_{3} m_{3}}^{* S Z} a_{l_{4} m_{4}}^{S Z}\right\rangle^{\mathrm{G}}=\sum_{l_{1}^{\prime}} \frac{2^{3}}{\pi^{3}} \int d r_{1} \cdots \int d r_{4} \int k_{1}^{2} d k_{1} \int k_{2}^{2} d k_{2} \int k_{4}^{2} d k_{4} \\
& \times(g \dot{G} G)_{r_{1}}(g \dot{G} G)_{r_{2}} W^{\mathrm{sz}}\left(r_{3}\right) W^{\mathrm{sz}}\left(r_{4}\right) P_{\delta \delta}^{\operatorname{lin}}\left(k_{1}\right) P_{g \Pi}\left(k_{2}\right) P_{g \Pi}\left(k_{4}\right) \\
& \times \frac{j_{l_{1}^{\prime}}^{\prime}\left(k_{1} r_{1}\right)}{k_{1}} j_{l_{3}}\left(k_{2} r_{1}\right) \frac{j_{l_{1}^{\prime}}^{\prime}\left(k_{1} r_{2}\right)}{k_{1}} j_{l_{4}}\left(k_{4} r_{2}\right) j_{l_{3}}\left(k_{2} r_{3}\right) j_{l_{4}}\left(k_{4} r_{4}\right) I_{l_{1} l_{1}^{\prime} l_{3}}^{m^{*} m_{1}^{\prime *} m_{3}^{*}}(\hat{\mathbf{n}}) I_{l_{2} l_{1}^{\prime} l_{4}}^{m_{2}^{*} m_{1}^{\prime *} m_{4}^{*}}(\hat{\mathbf{m}}) .
\end{aligned}
$$

Using this term, we can now write the Gaussian piece of the cross-correlation power between the squared temperatures between SZ and DSZ as 
$\left\langle a_{l m}^{\mathrm{kSZ}} a_{l^{\prime} m^{\prime}}^{* S Z^{2}}\right\rangle^{\mathrm{G}}=C_{l}^{\mathrm{G}} \delta_{l, l^{\prime}} \delta m, m^{\prime}$

$$
\begin{aligned}
= & \sum_{l_{1} m_{1} l_{2} m_{2}} \sum_{l_{3} m_{3} l_{4} m_{4}} \sum_{l_{1}^{\prime}} \frac{2^{3}}{\pi^{3}} \int d r_{1} \cdots \int d r_{4} \int k_{1}^{2} d k_{1} \int k_{2}^{2} d k_{2} \int k_{4}^{2} d k_{4}(g \dot{G} G)_{r_{1}}(g \dot{G} G)_{r_{2}} W^{\mathrm{sz}}\left(r_{3}\right) W^{\mathrm{sz}}\left(r_{4}\right) \\
& \times P_{\delta \delta}^{\operatorname{lin}}\left(k_{1}\right) P_{g \Pi}\left(k_{2}\right) P_{g \Pi}\left(k_{4}\right) \frac{j_{l_{1}^{\prime}}^{\prime}\left(k_{1} r_{1}\right)}{k_{1}} j_{l_{3}}\left(k_{2} r_{1}\right) \frac{j_{l_{1}^{\prime}}^{\prime}\left(k_{1} r_{2}\right)}{k_{1}} j_{l_{4}}\left(k_{4} r_{2}\right) j_{l_{3}}\left(k_{2} r_{3}\right) j_{l_{4}}\left(k_{4} r_{4}\right) \\
& \times I_{l_{1} l_{1}^{\prime} l_{3}}^{m_{1}^{*} m_{1}^{\prime *} m_{3}^{*}}(\hat{\mathbf{n}}) I_{l_{2} l_{1}^{\prime} l_{4}}^{m_{2}^{*} m_{1}^{\prime} * m_{4}^{*}}(\hat{\mathbf{m}}) I_{l l_{1} l_{2}}^{m^{*} m_{1} m_{2}^{*}}(\hat{\mathbf{n}}) I_{l^{\prime} l_{3} l_{4}}^{m^{\prime} m_{3}^{*} m_{4}}(\hat{\mathbf{m}}) .
\end{aligned}
$$

The last four integrals lead to a Wigner $6 j$ symbol:

$$
\sum_{m_{1} m_{2} m_{3} m_{4}}\left(\begin{array}{ccc}
l_{1} & l_{1}^{\prime} & l_{3} \\
m_{1} & m_{1}^{\prime} & m_{3}
\end{array}\right)\left(\begin{array}{ccc}
l_{2} & l_{1}^{\prime} & l_{4} \\
m_{2} & m_{1}^{\prime} & m_{4}
\end{array}\right)\left(\begin{array}{ccc}
l & l_{1} & l_{2} \\
m & m_{1} & m_{2}
\end{array}\right)\left(\begin{array}{ccc}
l^{\prime} & l_{3} & l_{4} \\
m^{\prime} & m_{3} & m_{4}
\end{array}\right)=\frac{\delta_{l l^{\prime}} \delta_{m m^{\prime}}}{2 l+1}\left\{\begin{array}{ccc}
l_{4} & l_{2} & l \\
l_{1} & l_{3} & l_{1}^{\prime}
\end{array}\right\} .
$$

Thus, simplifying, we obtain

$$
\begin{aligned}
C_{l}^{\mathrm{G}}= & \sum_{l_{1} l_{2} l_{3} l_{4} l_{1}^{\prime}} \frac{\prod_{i=1}^{4}\left(2 l_{i}+1\right)\left(2 l_{1}^{\prime}+1\right)}{4 \pi}\left\{\begin{array}{ccc}
l_{4} & l_{2} & l \\
l_{1} & l_{3} & l_{1}^{\prime}
\end{array}\right\}\left(\begin{array}{ccc}
l_{1} & l_{1}^{\prime} & l_{3} \\
0 & 0 & 0
\end{array}\right)\left(\begin{array}{ccc}
l_{2} & l_{1}^{\prime} & l_{4} \\
0 & 0 & 0
\end{array}\right)\left(\begin{array}{ccc}
l & l_{1} & l_{2} \\
0 & 0 & 0
\end{array}\right)\left(\begin{array}{ccc}
l & l_{3} & l_{4} \\
0 & 0 & 0
\end{array}\right) \\
& \times \frac{2^{3}}{\pi^{3}} \int d r_{1} \cdots \int d r_{4} \int k_{1}^{2} d k_{1} \int k_{2}^{2} d k_{2} \int k_{4}^{2} d k_{4}(g \dot{G} G)_{r_{1}}(g \dot{G} G)_{r_{2}} W^{\mathrm{sz}}\left(r_{3}\right) W^{\mathrm{sz}}\left(r_{4}\right) P_{\delta \delta}^{\mathrm{lin}}\left(k_{1}\right) P_{g \Pi}\left(k_{2}\right) P_{g \Pi}\left(k_{4}\right) \\
& \times \frac{j_{l_{1}^{\prime}}^{\prime \prime}\left(k_{1} r_{1}\right)}{k_{1}} j_{l_{3}}\left(k_{2} r_{1}\right) \frac{j_{l_{1}^{\prime}}^{\prime \prime}\left(k_{1} r_{2}\right)}{k_{1}} j_{l_{4}}\left(k_{4} r_{2}\right) j_{l_{3}}\left(k_{2} r_{3}\right) j_{l_{4}}\left(k_{4} r_{4}\right) .
\end{aligned}
$$

Note that there is an additional term, due to a permutation, which involves by interchanging $l_{3}$ and $l_{4}$ with $l_{1}^{\prime}$, respectively.

Similar to the Limber approximation used with the derivation of the Doppler SZ power spectrum, we can integrate over Bessel functions and simplify to obtain

$$
\begin{aligned}
C_{l}^{\mathrm{G}}= & \sum_{l_{1} l_{2} l_{3} l_{4} l_{1}^{\prime}} \frac{\prod_{i=1}^{4}\left(2 l_{i}+1\right)\left(2 l_{1}^{\prime}+1\right)}{4 \pi}\left\{\begin{array}{ccc}
l_{4} & l_{2} & l \\
l_{1} & l_{3} & l_{1}^{\prime}
\end{array}\right\}\left(\begin{array}{ccc}
l_{1} & l_{1}^{\prime} & l_{3} \\
0 & 0 & 0
\end{array}\right)\left(\begin{array}{ccc}
l_{2} & l_{1}^{\prime} & l_{4} \\
0 & 0 & 0
\end{array}\right)\left(\begin{array}{ccc}
l & l_{1} & l_{2} \\
0 & 0 & 0
\end{array}\right)\left(\begin{array}{ccc}
l & l_{3} & l_{4} \\
0 & 0 & 0
\end{array}\right) \\
& \times \frac{2}{\pi} \int \frac{d r_{1}}{d_{A}^{2}} \int \frac{d r_{2}}{d_{A}^{2}} \int k_{1}^{2} d k_{1}(g \dot{G})_{r_{1}}(g \dot{G})_{r_{2}} W^{\mathrm{sz}}\left(r_{1}\right) W^{\mathrm{sz}}\left(r_{2}\right) \times P_{\delta \delta}^{\operatorname{lin}}\left(k_{1}\right) P_{g \Pi}\left(\frac{l_{3}}{d_{A}} ; r_{1}\right) P_{g \Pi}\left(\frac{l_{4}}{d_{A}} ; r_{2}\right) \frac{j_{l_{1}^{\prime}}^{\prime}\left(k_{1} r_{1}\right)}{k_{1}} \frac{j_{l_{1}^{\prime}}^{\prime}\left(k_{1} r_{2}\right)}{k_{1}} .
\end{aligned}
$$

The non-Gaussian piece takes a similar form. First we introduce the trispectrum of pressure-pressure-baryon-baryon fluctuations and the power spectrum of velocity correlations in Eq. (97), and, to simplify, we expand the delta function associated with the trispectrum to two separate triangular parts, 


$$
\begin{aligned}
\delta_{D}\left(\mathbf{k}_{2}+\mathbf{k}_{4}+\mathbf{k}_{5}+\mathbf{k}_{6}\right) & =\int \frac{d^{3} \mathbf{k}^{\prime}}{(2 \pi)^{3}}(2 \pi)^{3} \delta_{D}\left(\mathbf{k}_{2}+\mathbf{k}_{4}+\mathbf{k}^{\prime}\right) \delta_{D}\left(\mathbf{k}_{5}+\mathbf{k}_{6}-\mathbf{k}^{\prime}\right) \\
& =\int \frac{d^{3} \mathbf{x}_{1}}{(2 \pi)^{3}} e^{i \mathbf{x}_{1} \cdot\left(\mathbf{k}_{2}+\mathbf{k}_{4}+\mathbf{k}^{\prime}\right)} \int \frac{d^{3} \mathbf{x}_{2}}{(2 \pi)^{3}} e^{i \mathbf{x}_{2} \cdot\left(\mathbf{k}_{5}+\mathbf{k}_{6}-\mathbf{k}^{\prime}\right)}
\end{aligned}
$$

The assumption here is that through this vector expansion, the vectorial representation of the quadrilateral formed by the trispectrum can be expressed through a vectorial configuration of two triangles involving two sides and the diagonal, respectively. With this, the trispectrum is expressed to be dependent only on the magnitude of the vectors ( $\left.\mathbf{k}_{2}, \mathbf{k}_{4}, \mathbf{k}_{5}, \mathbf{k}_{6}\right)$ and not on their directions. Using the Rayleigh expansion [Eq. (71)] above, we simplify to obtain

$$
\begin{aligned}
& \left\langle a_{l_{1} m_{1}}^{\mathrm{kSZ}} a_{l_{2} m_{2}}^{* \mathrm{kSZ}} a_{l_{3} m_{3}}^{* S Z} a_{l_{4} m_{4}}^{S Z}\right\rangle^{\mathrm{NG}} \\
& =\frac{(4 \pi)^{14}}{9} \int d r_{1} \cdots \int d r_{4} \sum_{l_{1}^{\prime} m_{1}^{\prime} l_{1}^{\prime \prime} m_{1}^{\prime \prime} m_{1}^{\prime \prime \prime} l_{2}^{\prime} m_{2}^{\prime} l_{2}^{\prime \prime} m_{2}^{\prime \prime} m_{2}^{\prime \prime \prime}} \sum_{L M} \times \int \frac{d^{3} \mathbf{k}_{1}}{(2 \pi)^{3}} \int \frac{d^{3} \mathbf{k}_{2}}{(2 \pi)^{3}} \int \frac{d^{3} \mathbf{k}_{4}}{(2 \pi)^{3}} \cdots \int \frac{d^{3} \mathbf{k}_{6}}{(2 \pi)^{3}} \int \frac{d^{3} \mathbf{k}^{\prime}}{(2 \pi)^{3}} \\
& \times i^{l_{1}^{\prime}+l_{3}+l_{4}+L}(-i)^{l_{2}^{\prime}+l_{3}+l_{4}-L} i_{1}^{l_{1}^{\prime \prime}+l_{2}^{\prime \prime}+l_{3}+l_{4}}(g \dot{G} G)_{r_{1}}(g \dot{G} G)_{r_{2}} W^{\mathrm{sz}}\left(r_{3}\right) W^{\mathrm{sz}}\left(r_{4}\right) P_{\delta \delta}^{\operatorname{lin}}\left(k_{1}\right) T_{\Pi g \Pi g}\left(k_{2}, k_{4}, k_{5}, k_{6}\right) \\
& \times \frac{j_{l_{1}^{\prime}}\left(k_{1} r_{1}\right)}{k_{1}} j_{l_{1}^{\prime \prime}}\left(k_{2} r_{1}\right) \frac{j_{l_{2}^{\prime}}\left(k_{1} r_{2}\right)}{k_{1}} j_{l_{2}^{\prime \prime}}\left(k_{4} r_{2}\right) j_{l_{3}}\left(k_{2} r_{3}\right) j_{l_{4}}\left(k_{4} r_{4}\right) \int x_{1}^{2} d x_{1} j_{l_{1}^{\prime \prime}}\left(k_{2} x_{1}\right) j_{l_{2}^{\prime \prime}}\left(k_{4} x_{1}\right) j_{L}\left(k^{\prime} x_{1}\right) \\
& \times \int x_{2}^{2} d x_{2} j_{l_{3}}\left(k_{5} x_{2}\right) j_{l_{4}}\left(k_{6} x_{2}\right) j_{L}\left(k^{\prime} x_{2}\right) Y_{l_{1}^{\prime}}^{m_{1}^{\prime}}\left(\hat{\mathbf{k}}_{1}\right) Y_{1}^{m_{1}^{\prime \prime \prime}}\left(\hat{\mathbf{k}}_{1}\right) Y_{l_{2}^{\prime}}^{m_{2}^{\prime}}\left(\hat{\mathbf{k}}_{1}\right) Y_{1}^{m_{2}^{\prime \prime \prime}}\left(\hat{\mathbf{k}}_{1}\right) I_{l_{1}^{\prime \prime} l_{2}^{\prime \prime} L}^{m_{1}^{\prime \prime} m_{2}^{\prime \prime} M}\left(\hat{\mathbf{x}}_{1}\right) I_{l_{3} l_{4} L}^{m_{3} m_{4} M}\left(\hat{\mathbf{x}}_{2}\right) \\
& \times I_{l_{1} l_{1}^{\prime} l_{1}^{\prime \prime} 1}^{m^{*} m_{1}^{\prime} * m_{1}^{\prime \prime} * m_{1}^{\prime \prime \prime} *}(\hat{\mathbf{n}}) I_{l_{2} l_{2}^{\prime} l_{2}^{\prime \prime} 1}^{m^{*} m^{\prime} * m_{2}^{\prime \prime} * m_{2}^{\prime \prime \prime} *}(\hat{\mathbf{m}}) \text {. }
\end{aligned}
$$

Following our derivation of the DSZ power spectrum, and employing the Limber approximation on the Bessel functions, we can simplify further and write the non-Gaussian piece of the correlation between the squared temperatures of SZ thermal and SZ Doppler effects as

$$
\begin{aligned}
& \left\langle a_{l m}^{\mathrm{kSZ}} a_{l^{\prime} m^{\prime}}^{* S Z^{2}}\right\rangle^{\mathrm{NG}}=C_{l}^{\mathrm{NG}} \delta_{l, l^{\prime}} \delta m, m^{\prime}
\end{aligned}
$$

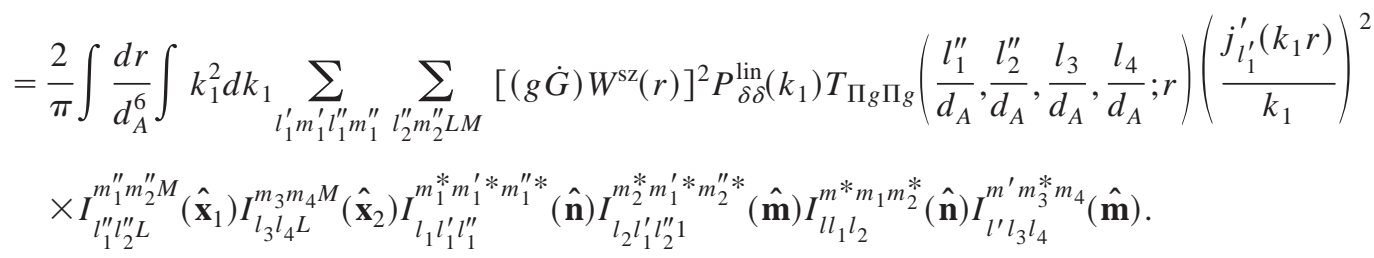

Following simplifications used in the case of the Gaussian part, we find

$$
\begin{aligned}
& C_{l}^{\mathrm{NG}}=\sum_{l_{1} l_{2} l_{3} l_{4} l_{1}^{\prime} l_{1}^{\prime \prime} l_{2}^{\prime \prime}} \frac{\prod_{i=1}^{4}\left(2 l_{i}+1\right) \prod_{j=\prime^{\prime}}^{\prime \prime \prime}\left(2 l_{1}^{j}+1\right)}{(4 \pi)^{3}}\left(\begin{array}{ccc}
l_{1} & l_{1}^{\prime} & l_{1}^{\prime \prime} \\
0 & 0 & 0
\end{array}\right)\left(\begin{array}{ccc}
l_{2} & l_{1}^{\prime} & l_{2}^{\prime \prime} \\
0 & 0 & 0
\end{array}\right)\left(\begin{array}{ccc}
l & l_{1} & l_{2} \\
0 & 0 & 0
\end{array}\right)\left(\begin{array}{ccc}
l & l_{1}^{\prime \prime} & l_{2}^{\prime \prime} \\
0 & 0 & 0
\end{array}\right) \\
& \times\left(\begin{array}{ccc}
l & l_{3} & l_{4} \\
0 & 0 & 0
\end{array}\right)^{2}\left\{\begin{array}{ccc}
l_{1} & l_{1}^{\prime} & l \\
l_{2}^{\prime \prime} & l_{1}^{\prime \prime} & l_{2}
\end{array}\right\} \frac{2}{\pi} \int \frac{d r}{d_{A}^{6}}\left[(g \dot{G}) W^{\mathrm{sz}}(r)\right]^{2} T_{\Pi g \Pi g}\left(\frac{l_{1}^{\prime \prime}}{d_{A}}, \frac{l_{2}^{\prime \prime}}{d_{A}}, \frac{l_{3}}{d_{A}}, \frac{l_{4}}{d_{A}} ; r\right) \int k_{1}^{2} d k_{1} P_{\delta \delta}^{\operatorname{lin}}\left(k_{1}\right)\left(\frac{j_{l_{1}^{\prime}}^{\prime}\left(k_{1} r\right)}{k_{1}}\right)^{2} .
\end{aligned}
$$


Note that the total contribution to the thermal $S Z^{2}-$ Doppler $S Z^{2}$ power spectrum is

$$
C_{l}^{2 \mathrm{kSZ}-\mathrm{SZ}}=C_{l}^{\mathrm{G}}+C_{l}^{\mathrm{NG}} .
$$

Since the full calculation of the squared power spectra is computationally time consuming, we make several simplifications as outlined in the next subsection. These simplifications make use of the fact that at small angular scales, we can utilize the flat-sky approximation, at the same scales, the velocity field is completely independent of the baryon field itself.

Under the all-sky approach, the end result, however, is numerically cumbersome since it involves a five-dimensional sum over a Wigner $6 j$ symbol (see Ref. [46] for a derivation). We introduce the flat-sky approach now, and simplify it further in the limit that the velocity field is independent of the baryon fluctuations.

\section{Flat-sky approach}

Following our previous definitions, we define the flat sky temperature squared power spectrum as

$$
\left\langle\Theta^{2 \mathrm{kSZ}}(\mathbf{l}) \Theta^{2 \mathrm{SZ}}\left(\mathbf{l}^{\prime}\right)\right\rangle=(2 \pi)^{2} \delta_{D}\left(\mathbf{l}+\mathbf{l}^{\prime}\right) C_{l}^{2 \mathrm{kSZ}-\mathrm{SZ}},
$$

where the Fourier transform of the squared temperature can be written as a convolution of the temperature transforms

$$
\Theta^{2}(\mathbf{l})=\int \frac{d^{2} \mathbf{l}_{1}}{(2 \pi)^{2}} \Theta\left(\mathbf{l}_{1}\right) \Theta\left(\mathbf{l}-\mathbf{l}_{1}\right) .
$$

Here it should be understood that $\Theta^{2}(\mathbf{l})$ refers to the Fourier transform of the square of the temperature rather than square of the Fourier transform of the temperature: $[\Theta(\mathbf{l})]^{2}$. To compute the square of the SZ thermal and SZ kinetic temperature power spectra, we take

$$
\begin{aligned}
\left\langle\Theta^{2 \mathrm{kSZ}}(\mathbf{l}) \Theta^{2 \mathrm{SZ}}\left(\mathbf{l}^{\prime}\right)\right\rangle= & (2 \pi)^{2} \delta_{D}\left(\mathbf{l}+\mathbf{l}^{\prime}\right) C_{l} \\
= & \int \frac{d^{2} \mathbf{l}_{1}}{(2 \pi)^{2}} \int \frac{d^{2} \mathbf{l}_{2}}{(2 \pi)^{2}} \\
& \times\left\langle\Theta^{\mathrm{kSZ}}\left(\mathbf{l}_{1}\right) \Theta^{\mathrm{kSZ}}\left(\mathbf{l}-\mathbf{l}_{1}\right)\right. \\
& \left.\times \Theta^{\mathrm{SZ}}\left(\mathbf{l}_{2}\right) \Theta^{\mathrm{SZ}}\left(\mathbf{l}^{\prime}-\mathbf{l}_{2}\right)\right\rangle .
\end{aligned}
$$

Note that the Fourier transform of the temperature fluctuations in the flat sky is

$$
\Theta(\mathbf{l})=\int d^{2} \theta e^{-i \mathbf{l} \cdot \theta} T(\theta) .
$$

In the small scale limit, where the density field is separated from the velocity field, we can separate the above cumulant into two parts, one involving just the power spectra of the pressure-baryon cross-correlation, and the other with the trispectrum of pressure and baryon fields. We first write the Gaussian-like piece as

$$
\begin{aligned}
& \left\langle\Theta^{\mathrm{kSZ}}\left(\mathbf{l}_{1}\right) \Theta^{\mathrm{kSZ}}\left(\mathbf{l}-\mathbf{l}_{1}\right) \Theta^{\mathrm{SZ}}\left(\mathbf{l}_{2}\right) \Theta^{\mathrm{SZ}}\left(\mathbf{l}^{\prime}-\mathbf{l}_{2}\right)\right\rangle^{\mathrm{G}} \\
& =\int \frac{d r_{1}}{d_{A}^{4}} \int \frac{d r_{2}}{d_{A}^{4}}\left[g\left(r_{1}\right) W^{\mathrm{SZ}}\left(r_{2}\right)\right]^{2} \frac{1}{3} v_{\mathrm{rms}}^{2} \\
& \left.\quad \times \int \frac{d k_{1}}{(2 \pi)} \int \frac{d k_{2}}{(2 \pi)} e^{i\left(k_{1} r_{1}+k_{2} r_{2}\right)} / \delta_{g}\left[\frac{\mathbf{l}_{1}}{d_{A}}, k_{1}\right] \delta_{\Pi}\left[\frac{\mathbf{l}_{2}}{d_{A}}, k_{2}\right]\right\rangle \\
& \quad \times \int \frac{d k_{3}}{(2 \pi)} \int \frac{d k_{4}}{(2 \pi)} e^{i\left(k_{3} r_{1}+k_{4} r_{2}\right)} \\
& \quad \times\left\langle\delta_{g}\left[\frac{\mathbf{l}-\mathbf{l}_{1}}{d_{A}}, k_{3}\right] \delta_{\Pi}\left[\frac{\mathbf{l}^{\prime}-\mathbf{l}_{2}}{d_{A}}, k_{4}\right]\right\rangle
\end{aligned}
$$

where we have taken $\left\langle(\hat{\theta} \cdot \mathbf{v})\left(\hat{\theta}^{\prime} \cdot \mathbf{v}^{\prime}\right)\right\rangle \sim 1 / 3 v_{\text {rms }}^{2}$, with $1 / 3$ coming from the fact that only a third of the velocity component contributes to the line of sight rms. We can now introduce power spectra in the above correlators such that

$$
\begin{aligned}
& \left\langle\Theta^{\mathrm{kSZ}}\left(\mathbf{l}_{1}\right) \Theta^{\mathrm{kSZ}}\left(\mathbf{l}-\mathbf{l}_{1}\right) \Theta^{\mathrm{SZ}}\left(\mathbf{l}_{2}\right) \Theta^{\mathrm{SZ}}\left(\mathbf{l}^{\prime}-\mathbf{l}_{2}\right)\right\rangle^{\mathrm{G}} \\
& =\int \frac{d r_{1}}{d_{A}^{4}} \int \frac{d r_{2}}{d_{A}^{4}}\left[g\left(r_{1}\right) W^{\mathrm{SZ}}\left(r_{2}\right)\right]^{2} \frac{1}{3} v_{\mathrm{rms}}^{2} \\
& \times \int \frac{d k_{1}}{(2 \pi)} e^{i k_{1}\left(r_{1}-r_{2}\right)}(2 \pi)^{2} \\
& \times \delta_{D}\left(\frac{\mathbf{l}_{1}}{d_{A}}+\frac{\mathbf{l}_{2}}{d_{A}}\right) P_{g \Pi}\left[\sqrt{\frac{l_{1}^{2}}{r_{1}^{2}}+k_{1}^{2}}\right] \\
& \times \int \frac{d k_{3}}{(2 \pi)} e^{i k_{3}\left(r_{1}-r_{2}\right)}(2 \pi)^{2} \delta_{D}\left(\frac{\mathbf{l}-\mathbf{l}_{1}}{d_{A}}+\frac{\mathbf{l}^{\prime}-\mathbf{l}_{2}}{d_{A}}\right) \\
& \times P_{g \Pi}\left[\sqrt{\frac{\left|l-l_{1}\right|^{2}}{r_{1}^{2}}+k_{3}^{2}}\right] .
\end{aligned}
$$

The integrals over the line-of-sight wave vectors behave such that only perpendicular Fourier modes contribute to the projected field, such that $l^{2} / d_{A}^{2} \gg k^{2}$. This is the so-called Limber approximation [37]. Doing the integral over the wave vector, then, results in a delta function in $\left(r_{1}-r_{2}\right)$ such that only contributions come from the same redshift. Putting the correlator back in the power spectrum equation [Eq. (111)], we now obtain

$$
\begin{aligned}
C_{l}^{\mathrm{G}}= & \int \frac{d^{2} \mathbf{l}_{1}}{(2 \pi)^{2}} \int \frac{d r}{d_{A}^{4}}(g \dot{G})^{2} W^{\mathrm{sz}}(r)^{2} \frac{1}{3} v_{\mathrm{rms}}^{2} \\
& \times 2 P_{g \Pi}\left(\frac{l_{1}}{d_{A}} ; r\right) P_{g \Pi}\left(\frac{\left|\mathbf{l}-\mathbf{l}_{1}\right|}{d_{A}} ; r\right),
\end{aligned}
$$

where we have introduced a factor of 2 to account for the additional permutation involved in the baryon densitypressure correlation.

Similarly, the non-Gaussian piece follows as 


$$
\begin{aligned}
& C_{l}^{\mathrm{NG}}=\int \frac{d^{2} \mathbf{l}_{1}}{(2 \pi)^{2}} \int \frac{d^{2} \mathbf{l}_{2}}{(2 \pi)^{2}} \int \frac{d r}{d_{A}^{6}}(g \dot{G})^{2} W^{\mathrm{sz}}(r)^{2} \frac{1}{3} v_{\mathrm{rms}}^{2} \\
& \times T_{g \Pi g \Pi}\left[\left(\frac{\mathbf{l}_{1}}{d_{A}}\right),\left(\frac{\mathbf{l}-\mathbf{l}_{1}}{d_{A}}\right),\left(\frac{\mathbf{l}_{2}}{d_{A}}\right),\left(\frac{-\mathbf{l}-\mathbf{l}_{2}}{d_{A}}\right) ; r\right] .
\end{aligned}
$$

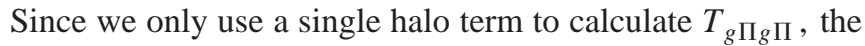
arguments are simply scalars and does not dependent on the orientation of the quadrilateral. In general, however, the trispectrum depends on the length of the four sides plus the orientation of at least one of the diagonals.

Comparing the flat sky power spectra written above and others derived under the all-sky assumption, we note that the two are related under similar approximations as the ones suggested for comparison between the all-sky and flat-sky Doppler SZ power spectra. For example, for the Gaussian piece, we can obtain the correspondence by setting $l_{1} \sim l_{4}$ and $l_{2}$ $\sim l_{3}$, and further simplifying to separate

$$
\begin{aligned}
C_{l}^{\mathrm{G}}= & \sum_{l_{3} l_{4}} \frac{\left(2 l_{3}+1\right)\left(2 l_{4}+1\right)}{4 \pi}\left(\begin{array}{ccc}
l & l_{3} & l_{4} \\
0 & 0 & 0
\end{array}\right)^{2} \\
& \times \int \frac{d r}{d_{A}^{4}}(g \dot{G} G)^{2} W^{\mathrm{sz}}(r)^{2} P_{g \Pi}\left(\frac{l_{3}}{d_{A}}\right) P_{g \Pi}\left(\frac{l_{4}}{d_{A}}\right) \frac{1}{3} v_{\mathrm{rms}}^{2} .
\end{aligned}
$$

This requires the sum

$$
\begin{gathered}
\sum_{l_{1} l_{2} l_{1}^{\prime}}\left(2 l_{1}+1\right)\left(2 l_{2}+1\right)\left(2 l_{1}^{\prime}+1\right)\left(\begin{array}{ccc}
l_{1} & l_{1}^{\prime} & l_{2} \\
0 & 0 & 0
\end{array}\right)^{2} \\
\times\left\{\begin{array}{lll}
l_{1} & l_{2} & l \\
l_{1} & l_{2} & l_{1}^{\prime}
\end{array}\right\}\left[j_{l_{1}^{\prime}}^{\prime}(k r)\right]^{2}=\frac{1}{3} .
\end{gathered}
$$

We note here that the above relation and the one suggested in Eq. (85) agree if we set the index associated with the Bessel function to either $l_{1}$ or $l_{2}$ such that the $l_{1}^{\prime}$ sum reduces to

$$
\sum_{l_{1}^{\prime}}\left(2 l_{1}^{\prime}+1\right)\left\{\begin{array}{lll}
l_{1} & l_{2} & l \\
l_{1} & l_{2} & l_{1}^{\prime}
\end{array}\right\}=1
$$

when $l_{1}, l_{2}$, and $l$ satisfy the triangular condition. This condition is, in fact, imposed by the Wigner $3 j$ symbol in above.

Finally, the correspondence between the flat sky ( $\mathbf{l}$ and $\mathbf{l}_{1}$ ) and all sky $\left(l, l_{3}\right.$, and $\left.l_{4}\right)$ approximations can be noted by introducing $\mathbf{l}_{3}=\mathbf{l}-\mathbf{l}_{1}$ and expanding the delta function of $\delta\left(\mathbf{l}-\mathbf{l}_{1}-\mathbf{l}_{3}\right)$ to obtain a Wigner $3 j$ squared symbol in $l, l_{1}$, and $l_{3}$ which correspond to $l, l_{3}$, and $l_{4}$ in the flat sky formulation. Such an expansion in flat-sky coordinates lead to the square of the Wigner $3 j$ symbol in the all-sky expression. We refer the reader to Ref. [5] for further details on such a reduction.

Following simplifications used in the case of the Gaussian part, we find the non-Gaussian piece to be

$$
\begin{aligned}
C_{l}^{\mathrm{NG}}= & \sum_{l_{1} l_{2} l_{3} l_{4}} \frac{\prod_{i=1}^{4}\left(2 l_{i}+1\right)}{(4 \pi)^{2}}\left(\begin{array}{ccc}
l & l_{1} & l_{2} \\
0 & 0 & 0
\end{array}\right)^{2}\left(\begin{array}{ccc}
l & l_{3} & l_{4} \\
0 & 0 & 0
\end{array}\right)^{2} \\
& \times \int \frac{d r}{d_{A}^{6}}\left[(g \dot{G}) W^{\mathrm{sz}}(r)\right]^{2} T_{\Pi g \Pi g} \\
& \times\left(\frac{l_{1}^{\prime \prime}}{d_{A}}, \frac{l_{2}^{\prime \prime}}{d_{A}}, \frac{l_{3}}{d_{A}}, \frac{l_{4}}{d_{A}} ; r\right) \frac{1}{3} v_{\mathrm{rms}}^{2} .
\end{aligned}
$$

We can obtain the correspondence between the flat-sky non-Gaussian expression and the all-sky non-Gaussian expression through a simplification as above for the Wigner $6 j$ symbol. To obtain the correspondence between $\mathbf{l}, \mathbf{l}_{1}$, and $\mathbf{l}_{2}$ in the flat-sky approximation and $l, l_{1}, l_{2}, l_{3}$ and $l_{4}$ in the allsky approximation, we can introduce $\mathbf{l}_{3}=\mathbf{l}-\mathbf{l}_{1}$ and $\mathbf{l}_{4}=\mathbf{l}+\mathbf{l}_{2}$ into the flat sky expression, and break the delta function formed by the flat sky trispectrum involving $\mathbf{l}_{1}+\mathbf{l}_{2}+\mathbf{l}_{3}+\mathbf{l}_{4}$ to two triangular parts involving two sides and the diagonal formed by $\mathbf{l}$ and expand these two. The two delta functions lead to the two squares of the Wigner $3 j$ symbol in the all-sky expression (see Ref. [5]).

\section{Signal-to-noise ratio}

In order to calculate the possibility for detection of the thermal $\mathrm{SZ}^{2}$-kinetic $\mathrm{SZ}^{2}$ power spectrum, we need the covariance of the estimator involved with the measurement of the squared power spectrum:

$$
\hat{C}_{l}^{2 \mathrm{kSZ}-\mathrm{SZ}}=\frac{A_{f}}{(2 \pi)^{2}} \int \frac{d^{2} \mathbf{l}}{A_{\mathrm{s}}} \Theta^{2 \mathrm{kSZ}}(\mathbf{l}) \Theta^{2 \mathrm{SZ}}(-\mathbf{l}) .
$$

Here $A_{\mathrm{s}}=\int d^{2} \mathbf{l}$ is the area in the two dimensional shell in Fourier space over which the integral is done, and $A_{f}$ is the total area of the survey in Fourier space and can be written as $A_{f}=(2 \pi)^{2} / \Omega$ with a total survey area on the sky of $\Omega$. Following Ref. [6], we can write down the covariance of our estimator as

$$
\operatorname{Cov}\left[\left(\hat{C}_{l}^{2 \mathrm{kSZ}-\mathrm{sZ}}\right)^{2}\right]=\frac{A_{f}}{A_{\mathrm{s}}}\left[\left(C_{l}^{2 \mathrm{kSZ}-\mathrm{SZ}}\right)^{2}+C_{l}^{2 \mathrm{kSZ}-\mathrm{kSZ}} C_{l}^{2 \mathrm{SZ}-\mathrm{sZ}}\right],
$$

where $C_{l}^{2 \mathrm{kSZ}-\mathrm{kSZ}}$ is the squared power spectrum of kinetic SZ and thermal SZ effects, while $C_{l}^{2 \mathrm{kSZ}-\mathrm{kSZ}}$ and $C_{l}^{2 \mathrm{SZ}-\mathrm{SZ}}$ are the squared power spectra of kinetic SZ and thermal SZ effects, respectively. Here we assume that squared fields are Gaussian. To calculate $C_{l}^{2 \mathrm{kSZ}-\mathrm{kSZ}}$ and $C_{l}^{2 \mathrm{SZ}-\mathrm{SZ}}$ we make several assumptions: We assume that the temperature squared power spectrum will be measured using two maps involving frequency separated SZ contribution (which will have $\Theta^{\mathrm{SZ}}$ $+\Theta^{\text {noise }}$ ) and a map with kinetic SZ contribution with the CMB primary component, such that they will be composed of $\Theta^{\text {primary }}+\Theta^{\mathrm{kSZ}}+\Theta^{\text {noise' }}$. Following such a separation, we can write the $C_{l}^{2 \mathrm{SZ}-\mathrm{SZ}}$ as 


$$
\begin{aligned}
\left\langle\Theta^{2 \mathrm{SZ}}(\mathbf{l}) \Theta^{2 \mathrm{SZ}}\left(\mathbf{l}^{\prime}\right)\right\rangle= & (2 \pi)^{2} \delta_{D}\left(\mathbf{l}+\mathbf{l}^{\prime}\right) C_{l}^{2 \mathrm{SZ}-\mathrm{SZ}} \\
= & \int \frac{d \mathbf{l}_{1}}{(2 \pi)^{2}} \int \frac{d \mathbf{l}_{2}}{(2 \pi)^{2}}\left\langle\Theta^{\mathrm{SZ}}\left(\mathbf{l}_{1}\right) \Theta^{\mathrm{SZ}}\left(\mathbf{l}-\mathbf{l}_{1}\right)\right. \\
& \left.\times \Theta^{\mathrm{SZ}}\left(\mathbf{l}_{2}\right) \Theta^{\mathrm{SZ}}\left(\mathbf{l}^{\prime}-\mathbf{l}_{2}\right)\right\rangle \\
= & \int \frac{d \mathbf{l}_{1}}{(2 \pi)^{2}}\left[2 C_{l_{1}}^{\mathrm{SZ}} C_{\left|\mathbf{l}-\mathbf{l}_{1}\right|}^{\mathrm{SZ}}\right. \\
& \left.+\int \frac{d \mathbf{l}_{2}}{(2 \pi)^{2}} T^{\mathrm{SZ}}\left(\mathbf{l}_{1}, \mathbf{l}-\mathbf{l}_{1}, \mathbf{l}_{2},-\mathbf{l}-\mathbf{l}_{2}\right)\right]
\end{aligned}
$$

and likewise for $C_{l}^{2 \mathrm{kSZ}-\mathrm{kSZ}}$. Here contributions come from a Gaussian part involving SZ power spectra and a nonGaussian part through the SZ trispectrum. Since the primary component fluctuations dominate the kinetic SZ temperature, and that there is no measurable trispectrum for this component under current adiabatic CDM predictions, we ignore any non-Gaussian contribution to $C_{l}^{2 \mathrm{kSZ}-\mathrm{kSZ}}$ and write it as the one with the Gaussian part only. This assumption is also safe at small angular scales out to $l \sim 10^{4}$, under the halo model, when $\Theta^{\mathrm{kSZ}}>\Theta^{\text {primary }}$ since the kinetic SZ effect can be described for the most part using large scale correlations instead of the single halo term.

In Eq. (122), the ratio of $A_{\mathrm{s}} / A_{f}$ is the total number of modes that measures the squared power spectrum independently and can be approximated such that $A_{\mathrm{s}} / A_{f}=f_{\text {sky }}(2 l$ $+1)$. To calculate the signal-to-noise ratio involved in the detection of the squared temperature power spectrum, we consider an optimized estimator with a weighing factor $W_{l}$ such that

$$
\hat{Y}=\sum_{l} W_{l} \hat{C}_{l}^{2 \mathrm{kSZ}-\mathrm{SZ}}
$$

and write the signal-to-noise ratio as

$$
\frac{S}{N}=\left[\frac{\langle\hat{Y}\rangle^{2}}{\operatorname{Cov}\left(\hat{Y}^{2}\right)}\right]^{1 / 2}
$$

The weight $W_{l}$ that maximizes the signal-to-noise ratio is $W_{l}=C_{l}^{2 \mathrm{kSZ}-\mathrm{SZ}} / \operatorname{Cov}\left[\left(C_{l}^{2 \mathrm{kSZ}-\mathrm{SZ}}\right)^{2}\right][6]$, and we can write the required signal-to-noise ratio as

$$
\frac{S}{N}=\left[f_{\mathrm{sky}} \sum_{l}(2 l+1) \frac{\left(C_{l}^{2 \mathrm{kSZ}-\mathrm{SZ}}\right)^{2}}{\left(C_{l}^{2 \mathrm{kSZ}-\mathrm{SZ}}\right)^{2}+C_{l}^{2 \mathrm{kSZ}-\mathrm{kSZ}} C_{l}^{2 \mathrm{SZ}-\mathrm{SZ}}}\right]^{1 / 2} .
$$

\section{Discussion}

In Fig. 13(a), we show the power spectrum of squared temperatures for the SZ thermal and SZ kinetic effects using the halo term. Here we have separated the Gaussian and non-Gaussian contributions to the squared power spectrum.
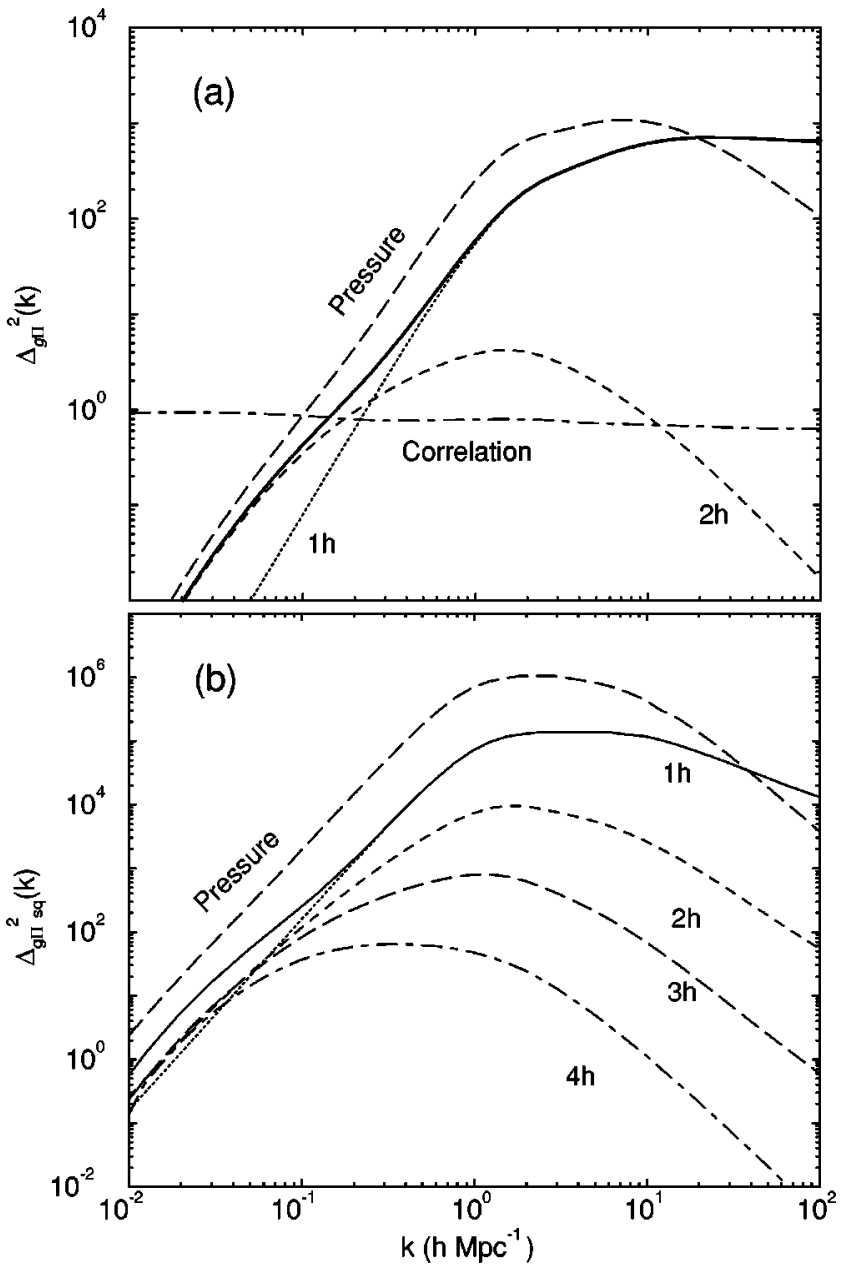

FIG. 12. The baryon-pressure (a) power spectrum (b) trispectrum $(z=0)$ broken into individual contributions under the halo description. The line labeled "correlation" shows the correlation coefficient of gas-pressure correlation with respect to gas-gas and pressure-pressure correlations. For reference, we also show the pressure power spectrum and the trispectrum.

As shown, the non-Gaussian contribution to the power spectrum is significantly higher than the Gaussian contributions.

The Gaussian contribution to the squared power spectrum traces the pressure-baryon density field power spectrum, which is shown in Fig. 12(a) using the halo model. In the same figure, for comparison, we also show the pressurepressure power spectrum and the correlation coefficient for the pressure-baryon spectrum with respect to the pressurepressure and baryon-baryon power spectra. The correlation behaves such that pressure and baryons trace each other at very large scales while the correlation is decreased at small scales due to the turnover in the pressure power spectrum. This is equivalent to the statement that there is no low mass halo contribution to the pressure power spectrum; these halos continue to contribute to the baryon density field power spectrum.

The non-Gaussian contribution to the thermal SZ-kinetic SZ squared temperature power spectrum traces the trispectrum formed by pressure and density field. We show this in Fig. 12(b) following the halo model. For comparison, we 


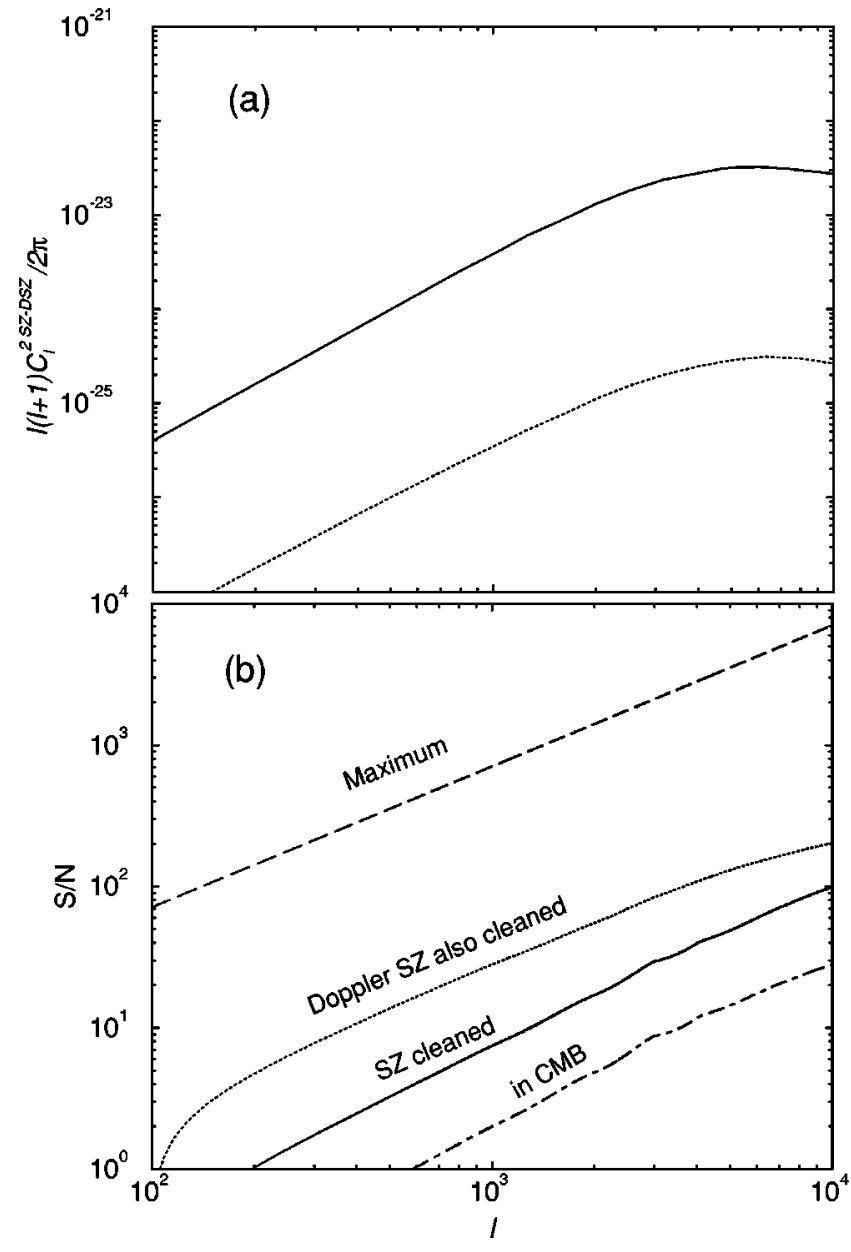

FIG. 13. (a) The SZ thermal-SZ kinetic power spectrum of squared temperatures. Here we show the contribution to the power spectrum when only Gaussian terms, i.e., power spectra of pressuredensity correlation, are considered (dotted line) and when nonGaussianities are introduced through the pressure-density trispectrum (solid line). In (b), we show the cumulative signal-to-noise ratio for the detection of the SZ thermal-SZ kinetic squared temperature power spectrum using information in multipoles from 2000 to 10000 and assuming no instrumental or any other noise contributions to the covariance. The signal-to-noise ratio is calculated assuming that the power spectrum is measured in CMB data (dotdashed line), with a perfect frequency cleaned SZ thermal map (solid line) and with a perfect SZ thermal and SZ kinetic effect maps (dotted line). In a dashed line, we show the maximum signalto-noise ratio achievable for the power spectrum, with only a Gaussian contribution to the covariance.

also show the trispectrum formed by pressure alone in the same figure. The pressure-baryon trispectrum is such that at large scales, corresponding to linear scales, significant contributions come from the correlations between halos instead of the single halo term. If there are significant contributions coming to the squared temperature power spectrum from such linear scales, the Gaussian part of the power spectrum should dominate. Since all contributions to the squared temperature power spectrum come from small angular scales corresponding to nonlinear scales in the pressure-baryon trispectrum, we only use the single halo contribution in cal- culating the non-Gaussian part of the squared temperature power spectrum. In both Gaussian and non-Gaussian parts of the power spectrum, the velocity field of the halos are taken to be the large scale bulk flows through the linear theory.

In order to assess the maximum possibility for a measurement of the temperature squared power spectrum involving kinetic SZ and thermal SZ effects, here we ignore the detector and beam noise contributions to the covariance. Also, we assume full-sky experiments with $f_{\text {sky }}=1$.

As written in Eq. (123), the contribution to the covariance comes as a convolution in Fourier space. Thus even at small angular scales corresponding to high multipoles, noise contributions can come from large angular scales or low wavelength modes. Such modes do not have any signal, and by only contributing to the variance they can reduce the effective signal-to-noise ratio in the measurement. Since the squared power spectrum effectively peaks at multipoles of $\sim 10^{4}$, we can essentially ignore any contribution to the signal, as well as noise, from multipoles of less than few thousand. These are the same multipoles in which the CMB primary anisotropies dominate, thereby increasing the effective noise for the measurement. In order to remove the low multipoles, we introduce a filtering scheme to the spherical or Fourier transform of the temperature anisotropy measurements, and suppress the low multipole data such that the filter essentially acts as a high pass filter above some $l$ $>l_{\min }$.

In Fig. 13(b), we show the cumulative signal to noise for the measurement of the thermal SZ-kinetic SZ squared temperature power spectrum. Here, we have assumed an experiment, with no instrumental noise, such that information is only used in multipoles of 2000-10000. We find a significant signal-to-noise ratio for the detection of the squared temperature power spectrum, especially when using a frequency cleaned SZ map with a CMB map, which has no SZ contribution. In fact one can use the CMB map itself, especially if multifrequency information is not available for SZ separation. Since an experiment with multipolar information out to $l \sim 10^{4}$ will not readily be available, we consider two separated realistic cases, involving a large angular scale experiment, similar to that of Planck, and a small angular scale experiment similar to the ones proposed for the study of SZ effect. We summarize our results in Fig. 14. As shown in Fig. 14(a), an experiment only sensitive to multipolar information ranging from 100 to 1500 does not have any signal-to-noise ratio for a detection of the squared power spectrum. With a perfect SZ separated map in this multipolar range, the cumulative signal-to-noise ratio for the squared power spectrum is in the order of $\sim 0.01$. Thus it is unlikely that Planck data will be useful for this study. Since we have not included any instrumental noise in calculating the signal-to-noise ratio, the realistic signal-to-noise ratio for Planck data would be even lower.

Going to smaller angular scales, we find that the signal increases significantly such that an experiment only sensitive to the range of $l \sim 2000$ to 5000 has an adequate signal-tonoise ratio for a detection of the squared power spectrum. A multifrequency experiment in the arcminute scales can use its frequency cleaned SZ map to cross-correlate with the 

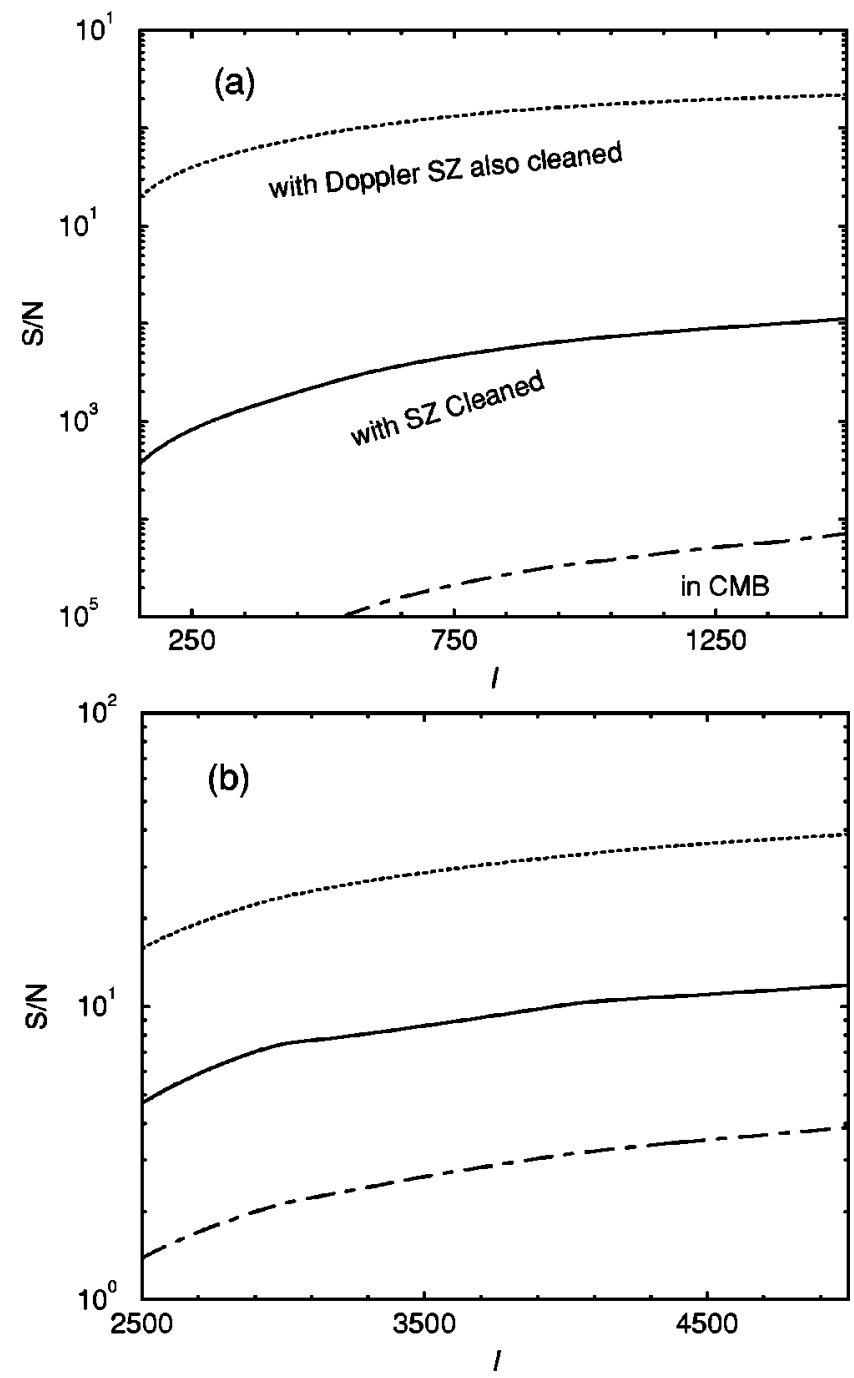

FIG. 14. The cumulative signal-to-noise ratio for the detection of the thermal SZ-kinetic SZ squared temperature power spectrum. In (a), we consider a large angular scale experiment, consistent with Planck measurements, and use multipole information from $l$ of 100 to 1500 . The cumulative signal-to-noise ratio, even with a perfectly cleaned SZ map in this multipole range, is significantly less than 1, suggesting that a detection is not possible. In (b), we show the cumulative signal-to-noise ratio for a small angular scale experiment with information in the multipole range of 2000 to 5000 . There is an adequate signal-to-noise ratio for the detection of the squared temperature power spectrum at such small scales even if the SZ effect is not completely separated from the thermal CMB contribution. For comparison, with to the dotted lines, we show the signal-to-noise ratio achievable if the kinetic SZ effect is separated from the CMB contribution, in addition to a SZ separated map.

CMB map and obtain the squared power spectrum with a cumulative signal-to-noise ratio on the order of a few tens. A comparison to Fig. 13(b) suggests that going to lower scales beyond 5000 increases the signal-to-noise ratio, and this is due to the fact that SZ thermal-SZ kinetic squared power spectrum peaks at multipoles of $\sim 7000$ to 8000 , suggesting that for an optimal detection of the squared power spectrum, one should also include observations out to such high multipoles.

\section{SUMMARY AND CONCLUSIONS}

We have discussed non-Gaussian effects associated with local large-scale structure contributions to the cosmic microwave background temperature fluctuations during the reionized epoch involving the thermal Sunyaev-Zel'dovich effect. At a low redshift, distribution functions associated with the large scale structure properties are non-Gaussian, primarily due to the nonlinear gravitational evolution. The nonGaussianities associated with the SZ effect is due to the nonGaussian distribution of the large scale pressure fluctuations. Since we do not have reliable analytical methods to calculate the higher order clustering of pressure or, as a matter of fact, any other property associated with large scale structure including the dark matter distribution, we have utilized the so-called halo approach to clustering. The basic description of this technique is that correlation functions, and their Fourier analogies, can be described through clustering of the property within and between halos. The halos themselves are assumed to be clustered with respect to the linear density field and with a mass dependent bias. We use linear theory and its perturbations out to second order to describe the clustering of halos. We use the halo method to describe clustering of pressure assuming that gas is in hydrostatic equilibrium with the dark matter density profile in halos. We use the virial equation to describe the electron temperatures.

It is well known that the frequency dependence of the SZ effect allows a separation of its contribution from other temperature fluctuations and foreground anisotropies in multifrequency $\mathrm{CMB}$ experiments. Using the pressure trispectrum, the Fourier analog of the four-point correlation function, calculated under the halo model, we discuss the full covariance of the SZ thermal power spectrum. The covariance, with the inclusion of non-Gaussianities, allows us to properly establish the errors associated with SZ power spectrum measurements. Thus we can use this full covariance to study the astrophysical uses of the SZ effect. Through a Fisher matrix formalism, we discuss how well a measurement of the SZ power spectrum can be used to conclude details on gas and temperature evolution and show that there are significant degeneracies between gas and temperature evolution parameters. The SZ power spectrum from a Planck mission can be used to understand the amount of baryons present in halos that contribute to the SZ effect and to establish any nongravitational heating of electrons, such as due to preheating with an error of $0.7 \mathrm{keV}$.

With the SZ thermal effect separated in temperature fluctuations using its frequency information, the kinetic SZ effect, also known as the Ostriker-Vishniac effect, is expected to dominate the thermal contribution at small angular scales corresponding to multipoles of few thousand. This effect arises from the baryon modulation of the first order Doppler effect. The presence of the SZ kinetic effect can be determined through a cross-correlation between the SZ thermal and a CMB map at small scales. Since the SZ kinetic effect is second order, however, contributions to such a crosscorrelation arise to lower order in the form of a three-point correlation function, or a bispectrum in Fourier space. We suggest an additional statistic that can be used to study the 
correlation between pressure traced by the SZ thermal effect and the baryons traced by the SZ kinetic effect involving the temperature anisotropy power spectrum of squared temperatures instead of the usual temperature itself. This power spectrum probes the trispectrum formed by the pressure-baryon cross-correlation. Through a signal-to-noise calculation, we show that future small angular scale multifrequency CMB experiments, sensitive to multipoles of a few thousand, will be able to measure the cross-correlation of SZ thermal and SZ kinetic effect through a temperature squared power spectrum.

\section{ACKNOWLEDGMENTS}

I am grateful to my advisor, Wayne $\mathrm{Hu}$, for suggesting problems and calculations presented here and in all our papers cowritten during the last two years. I thank the other thesis committee members, John Carlstrom, Scott Dodelson and Don York for their guidance and helpful suggestions. During the four years at Chicago, I was supported by individual grants to John Carlstrom and Don York and a GrantIn-Aid of Research from Sigma Xi, the National Science Honor Society.
[1] D.N. Spergel and D.M. Goldberg, Phys. Rev. D 59, 103001 (1999); D.M. Goldberg and D.N. Spergel, ibid. 59, 103002 (1999); M. Zaldarriaga and U. Seljak, ibid. 59, 123507 (1999); H. V. Peiris and D. N. Spergel, Astrophys J. 540, 605 (2000).

[2] A. Cooray and W. Hu, Astrophys. J. 534, 533 (2000).

[3] R.K. Sachs and A.M. Wolfe, Astrophys. J. 147, 73 (1967).

[4] U. Seljak, Astrophys. J. 463, 1 (1996).

[5] W. Hu, Phys. Rev. D 62, 043007 (2000).

[6] M. Zaldarriaga, Phys. Rev. D 62, 063510 (2000).

[7] R.A. Sunyaev and Ya.B. Zel'dovich, Mon. Not. R. Astron. Soc. 190, 413 (1980).

[8] J.E. Carlstrom, M. Joy, and L. Grego, Astrophys. J. Lett. 456, L75 (1996).

[9] M. Jones et al., Nature (London) 365, 320 (1993).

[10] A. Cooray, W. Hu, and M. Tegmark, Astrophys. J. Lett. 540, 1 (2000).

[11] P. de Bernardis et al., Nature (London) 404, 955 (2000).

[12] F.M. Persi, D.N. Spergel, R. Cen, and J.P. Ostriker, Astrophys. J. 442, 1 (1995); A.C. da Silva, D. Barbosa, A.R. Liddle, and P.A. Thomas, astro-ph/9907224; E. Komatsu and T. Kitayama, Astrophys. J. Lett. 526, L1 (1999); G.P. Holder, J.J. Mohr, J.E. Carlstrom, A.E. Evrard, and E.M. Leitch, Astrophys. J. 544, 629 (2000); A. Refregier, E. Komatsu, D.N. Spergel, and U.-L. Pen, Phys. Rev. D 61, 123001 (2000); G. P. Holder and J. E. Carlstrom (unpublished).

[13] U. Seljak, J. Burwell, and U.-L. Pen, Phys. Rev. D 63, 063001 (2001).

[14] V. Springel, M. White, and L. Hernquist, Astrophys. J. 549, 681 (2001).

[15] A. Refregier and R. Teyssier, astro-ph/0012086.

[16] A. Cooray, Phys. Rev. D 62, 103506 (2000).

[17] A. Meiksin and M. White, Mon. Not. R. Astron. Soc. 308, 1179 (1999); R. Scoccimarro, M. Zaldarriaga, and L. Hui, Astrophys. J. 527, 1 (1999).

[18] A. Cooray and W. Hu, Astrophys. J. 548, 7 (2001).

[19] J.P. Ostriker and E.T. Vishniac, Nature (London) 322, 804 (1986); E.T. Vishniac, Astrophys. J. 322, 597 (1987).

[20] W. Hu, Astrophys. J. 529, 12 (1999).

[21] D.J. Eisenstein and W. Hu, Astrophys. J. 511, 5 (1999).

[22] M.H. Goroff, B. Grinstein, S.-J. Rey, and M. Wise, Astrophys. J. 311, 6 (1986).

[23] J.N. Fry, Astrophys. J. 279, 499 (1984).

[24] P. J. E. Peebles, The Large-Scale Structure of the Universe (Princeton University Press, Princeton, 1980).
[25] P.T.P. Viana and A.R. Liddle, Mon. Not. R. Astron. Soc. 303, 535 (1999).

[26] E.F. Bunn and M. White, Astrophys. J. 480, 6 (1997).

[27] R.J. Scherrer and E. Bertschinger, Astrophys. J. 381, 349 (1991); R. Sheth and B. Jain, Mon. Not. R. Astron. Soc. 285, 231 (1997); U. Seljak, astro-ph/0001493; C.-P. Ma and J.N. Fry, Astrophys. J. 543, 503 (2000); R. Scoccimarro, R. Sheth, L. Hui, and B. Jain, astro-ph/0006319.

[28] A. Cooray and W. Hu, Astrophys. J. 554, 56 (2001).

[29] A. Cooray, W. Hu, and J. Miralda-Escudé, Astrophys. J. Lett. 535, L9 (2000).

[30] H.J. Mo, Y.P. Jing, and S.D.M. White, Mon. Not. R. Astron. Soc. 284, 189 (1997); H.J. Mo and S.D.M. White, ibid. 282, 347 (1996).

[31] J.P. Henry, Astrophys. J. 534, 565 (2000).

[32] J. Navarro, C. Frenk, and S.D.M. White, Astrophys. J. 462, 563 (1996).

[33] W.H. Press and P. Schechter, Astrophys. J. 187, 425 (1974); also see R.K. Sheth and B. Tormen, Mon. Not. R. Astron. Soc. 308, 119 (1999).

[34] J. S. Bullock, T. S. Kolatt, and Y. Sigad, Mon. Not. R. Astron. Soc. (to be published), astro-ph/9908159; Y.P. Jing, Astrophys. J. 535, 30 (2000).

[35] J.J. Mohr and A.E. Evrard, Astrophys. J. 491, 38 (1997).

[36] N. Makino, S. Sasaki, and Y. Suto, Astrophys. J. 497, 555 (1998); Y. Suto, S. Sasaki, and N. Makino, ibid. 509, 544 (1998).

[37] D. Limber, Astrophys. J. 119, 655 (1954).

[38] W. Hu and M. White, astro-ph/0010352.

[39] A.J.S. Hamilton, Mon. Not. R. Astron. Soc. 289, 285 (1999); A.J.S. Hamilton and M. Tegmark, ibid. 312, 285 (2000).

[40] L Knox, Phys. Rev. D 52, 4307 (1995); G. Jungman, M. Kamionkowski, A. Kosowsky, and D.N. Spergel, ibid. 54, 1332 (1995); J.R. Bond, G. Efstathiou, and M. Tegmark, Mon. Not. R. Astron. Soc. 291, L33 (1997); M. Zaldarriaga, D.N. Spergel, and U. Seljak, Astrophys. J. 488, 1 (1997); D.J. Eisenstein, W. Hu, and M. Tegmark, ibid. 518, 2 (1999).

[41] S. Majumdar, astro-ph/0102430; G. Holder and J. Carlstrom, in Microwave Foregrounds, edited by A. de Oliveira-Costa and M. Tegmark (ASP, San Francisco, 1999).

[42] N. Kaiser, Astrophys. J. 282, 374 (1984).

[43] L.M. Griffiths, D. Barbosa, and A.A.R. Liddle, Mon. Not. R. Astron. Soc. 308, 845 (1999). 
[44] Z. Haiman and L. Knox, in Microwave Foregrounds [41], astro-ph/9902311.

[45] S. Dodelson and J.M. Jubas, Astrophys. J. 439, 503 (1995); G. Efstathiou, in Large Scale Motions in the Universe. A Vatican Study Week, edited by V.C. Rubin and G.V. Coyne (Princeton
University Press, Princeton, 1988), p. 299; A.H. Jaffe and M. Kamionkowski, Phys. Rev. D 58, 043001 (1998).

[46] A. Cooray, Ph.D. thesis, University of Chicago, Chicago, 2001, available from the University of Chicago Crear Science Library or from the author. 